HEINSTEN FREDERICH LEAL DOS SANTOS

\title{
Controle ativo-passivo de vibrações estruturais usando materiais piezelétricos: Otimização e quantificação de incertezas
}

Tese apresentada à Escola de Engenharia de São Carlos da Universidade de São Paulo como parte dos requisitos para a obtenção do título de Doutor em Engenharia Mecânica.

Área de Concentração: Dinâmica de Máquinas e Sistemas 
AUTORIZO A REPRODUÇÃO TOTAL OU PARCIAL DESTE TRABALHO, POR QUALQUER MEIO CONVENCIONAL OU ELETRÔNICO, PARA FINS DE ESTUDO E PESQUISA, DESDE QUE CITADA A FONTE.

Leal dos Santos, Heinsten Frederich

Controle ativo-passivo de vibrações estruturais usando materiais piezelétricos: Otimização e quantificação de incertezas / Heinsten Frederich Leal dos Santos; orientador Prof. Dr. Marcelo Areias Trindade. São Carlos, 2012.

Tese (Doutorado) - Programa de Pós-Graduação em Engenharia Mecânica e Área de Concentração em Dinâmica de Máquinas e Sistemas -- Escola de Engenharia de São Carlos da Universidade de São Paulo, 2012.

1. Materiais piezelétricos. 2. Vibrações controle. 3. APPN. 4. Algoritmo genético. 5. Otimização. 6. Circuitos Shunt ativo-passivo. 7. Elementos Finitos. I. Título. 
FOLHA DE JULGAMENTO

Candidato: Licenciado HEINSTEN FREDERICH LEAL DOS SANTOS.

Título da tese: "Controle ativo-passivo de vibrações estruturais usando materiais piezelétricos: otimização e quantificação de incertezas".

Data da defesa: $14 / 11 / 2012$

Comissão Julgadora:

Resultado:

Prof. Associado Marcelo Areias Trindade (Orientador)

(Escola de Engenharia de São Carlos/EESC)

Prof. Dr. Carlos De Marqui Junior

(Escola de Engenharia de São Carlos/EESC)

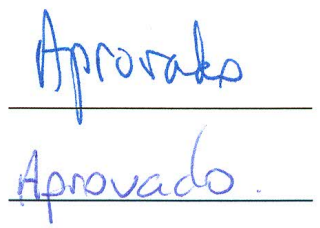

Prof. Dr. André Teófilo Beck

(Escola de Engenharia de São Carlos/EESC)

Prof. Dr. Antonio Marcos Gonçalves de Lima

(Universidade Federal de Uberlândia/UFU)

Prof. Dr. Vicente Lopes Junior

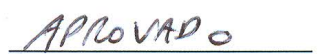

(Universidade Estadual Paulista "Júlio de Mesquita Filho"/UNESP- Ilha Solteira)

Coordenador do Programa de Pós-Graduação em Engenheira Mecânica:

Prof. Associado Marcelo Areias Trindade

Presidente da Comissão de Pós-Graduação:

Prof. Titular Denis Vinicius Coury 



\section{Agradecimentos}

- Agradeço primeiramente a Deus por iluminar os meus caminhos durante a realização desta jornada, sei que sem ELE manter os pés no chão nada disso seria possível.

- Agradeço a CAPES, CNPq pelo apoio financeiro.

- A nossa secretária Cristina, nossa mãe dentro do laboratório, sempre nos auxiliou e não deixando faltar nada para a realização deste trabalho.

- Aos técnicos do nosso laboratório, Sérgio, Diego e ao Xina, companheiro para sempre resolver nossas dúvidas e nos auxiliar prontamente nas dificuldades técnicas, meu muito obrigado.

- Aos meus companheiros que sempre estiveram presentes nesta jornada, Rafael Vinicius (BIG), Denise Marin, Hélio Sulleiman, Mário Pardo, Viviane Santana, Graciela, Eledir Leonardo, Ronaldo Junio e Paula. Sem nossas conversas, incentivos em momentos de desânimos, este caminho teria sido mais longo e árduo.

- Aos professores André Teófilo Beck, Paulo Sergio Varoto, Leopoldo de Oliveira da Escola de Engenharia de São Carlos, pelas trocas de idéias, pela convivência no laboratório. Saibam que todo ato por menor que seja, refletiu no bom andamento desta tese.

- Aos professores da banca pela disposição e presença nesta banca, conheço o trabalho desenvolvido pelos senhores e sei que este trabalho está sendo avaliado pelos melhores.

- Ao Professor/Orientador/Amigo Marcelo Areias Trindade pelos ensinamentos, confiança, dedicação durante o caminho deste doutorado, se cheguei onde estou foi porque tive o senhor como orientador dos passos dentro e fora desta instituição. Muito obrigado por tudo.

- Aos meus irmãos, Aline Carolina Leal dos Santos e Vinícius Guilherme Leal dos Santos por simplesmente existirem. Saibam que apesar das distâncias sei que sempre posso contar com vocês e espero que saibam que podem contar comigo sempre.

- A minha vó, Isabel Coelho, que sempre preocupada, reza todos os dias por cada um de nós. 
- Em especial aos meus pais, Maria Cristina Coelho Leal dos Santos e José Carlos Leal dos Santos, pessoas das quais sempre estiveram ao meu lado, nos momentos mais difíceis, mais complicados e sempre souberam olhar e dizer com uma calma e confiança que só os pais tem: "Erga a cabeça e dê mais um passo, um caminho por mais longo que possa ser... começa com um único passo". Muito obrigado... 
"Hoje, vocês são o novo, mas com o tempo envelhecerão e serão removidos. Não quero ser dramático, mas é uma verdade. O tempo de que vocês dispõem é limitado, e por isso não deveriam desperdiçá-lo vivendo a vida de outra pessoa. Não se deixem aprisionar por dogmas - isso significa viver sob os ditames do pensamento alheio. Não permitam que o ruído das outras vozes supere o sussurro de sua voz interior. E, acima de tudo, tenham a coragem de seguir seu coração e suas intuições, porque eles de alguma maneira já sabem o que vocês realmente desejam se tornar. Tudo mais é secundário."

Steve Jobs, CEO Apple, 1955-2011 


\section{Resumo}

SANTOS, H.F.L. Controle ativo-passivo de vibrações estruturais usando materiais piezelétricos: Otimização e Quantificação de Incertezas. 2012, Tese (Doutorado) - Escola de Engenharia de São Carlos, Universidade de São Paulo, São Carlos-SP.

Esta tese apresenta uma análise numérica do controle de vibrações estruturais através de cerâmicas piezelétricas em extensão conectadas a circuitos ativo-passivos compostos por resistência, indutância e fonte de tensão. Para tal, um modelo de elementos finitos de vigas sanduíche com três camadas elásticas e/ou piezelétricas foi desenvolvido. Realizou-se também uma modelagem dos componentes do circuito elétrico e seu acoplamento à estrutura gerando assim uma equação de movimento acoplada para a estrutura com elementos piezelétricos conectados aos circuitos elétricos. Uma análise harmônica das equações obtidas foi realizada para se obter uma avaliação preliminar dos efeitos causados pelos componentes elétricos do circuito na estrutura. Observou-se que os elementos passivos do circuito, resistência e indutância, tem não somente um efeito de absorvedor dinâmico de vibrações mas, também, promovem uma amplificação da autoridade de controle no caso de se atuar através da fonte de tensão. Usando a metodologia tradicional de projeto de absorvedores dinâmicos de vibrações, derivou-se expressões para os valores de resistência e indutância de modo a maximizar o desempenho passivo do sistema. Uma análise do efeito de incertezas das constantes piezelétricas e dielétricas da cerâmica piezelétrica considerada e dos componentes de resistência e indutância do circuito elétrico no desempenho do controle passivo e ativo-passivo de estrutura tipo viga cantilever foi realizada. O objetivo desta análise foi quantificar robustez e sensibilidade do controle proposto. Em sequida, um estudo de otimização dos valores de resistência e indutância do circuito elétrico em função da tensão elétrica de controle máxima a ser aplicada em uma placa com diversos atuadores piezelétricos foi realizado. Finalmente e também para a estrutura tipo placa, uma análise de incertezas da rigidez da cola na interface entre estrutura e atuadores piezelétricos e seus efeitos no desempenho do controle passivo e ativo-passivo foi realizada.

Palavras-chave: controle de vibrações; materiais piezelétricos; APPN; circuitos shunt ativo-passivos; elementos finitos; algoritmo genético; otimização; análise de incertezas. 


\section{Abstract}

SANTOS, H.F.L. Acitve-passive strucutural control using piezoelectric materials: optimization and uncertainty quantification. 2008, Doctoral Thesis - São Carlos School of Engineering, University of São Paulo, São Carlos-SP, Brazil.

This work presents a numerical analysis of the structural vibration control using piezoelectric materials in extension mode connected to active-passive electric circuits composed of resistance, inductance and voltage source. For that, a finite element model for sandwich beams with three elastic or piezoelectric layers was developed. A modeling of the electric circuit dynamics and its coupling to the structure with piezoelectric elements was also done. A harmonic analysis of the resulting equations was performed to yield a preliminary evaluation of the effects caused by the electric circuit components on the structure. It was observed that the passive circuit components not only lead to a dynamic vibration absorber effect but also to an amplification of the control authority in case of actuation using the voltage source. Using the standard methodology for the design of dynamic vibration absorbers, expressions were derived for the resistance and inductance values that optimize the passive vibration control performance of the system. An analysis of the effect of uncertainties of piezoelectric and dielectric constants of piezoelectric ceramic and resistance and inductance components of the shunt circuit on the passive and active-passive control performance for a cantilever beam structure was performed. The objective of this analysis was to quantify robustness and sensitivity of the proposed control. Then, an optimization study of the values of resistance and inductance of the shunt circuit as a function of the maximum control voltage to be applied on a plate with several piezoelectric actuators was performed. Finally and also for the plate structure, an analysis of uncertainties in the stiffness of the adhesive interface between structure and piezoelectric actuators and their effects on the performance of passive control and active-passive was performed.

Keywords: vibration control; piezoelectric materials; APPN; active-passive shunt circuits; finite elements; optimization; genetic algorithm; uncertainty analysis. 


\section{Sumário}

1 Introdução 1

1.1 Introdução . . . . . . . . . . . . . . . . . . . . . . . . . . 1

1.2 Revisão Bibliográfica . . . . . . . . . . . . . . . . . . 2

1.2.1 Materiais piezelétricos e seus modos de atuação . . . . . . . . . 2

1.2.2 Modelagem de estruturas piezelétricas . . . . . . . . . . . 5

1.2.3 Controle passivo de vibrações usando materiais piezelétricos . . . 7

1.2.4 Controle ativo de vibrações usando materiais piezelétricos . . . . 8

1.2.5 Controle ativo-passivo de vibrações usando materiais piezelétricos 10

1.2.6 Quantificação de incertezas e modelagem estocástica . . . . . . . 11

1.3 Objetivos . . . . . . . . . . . . . . . . . . 14

2 Modelo em elementos finitos de placas sanduíche piezelétricas 15

2.1 Hipóteses cinemáticas e equações constitutivas . . . . . . . . . . . . . 15

2.1.1 Deslocamentos e deformações . . . . . . . . . . . . . . . . 16

2.1.2 Equações constitutivas para materiais piezelétricos . . . . . . . 20

2.2 Formulação variacional . . . . . . . . . . . . . . . . . . . . . 23

2.3 Formulação em Elementos Finitos . . . . . . . . . . . . . . . . . 29

2.3.1 Discretização de deslocamentos e deformações . . . . . . . . . . . . . . 29

2.3.2 Discretização dos deslocamentos elétricos . . . . . . . . . . . 34

2.3.3 Discretização das expressões de trabalho virtual . . . . . . . . . . 35

2.4 Equações de movimento . . . . . . . . . . . . . . . . . 38

2.5 Validação do modelo proposto . . . . . . . . . . . . . . . . . 40

2.6 Pastilhas em Extensão . . . . . . . . . . . . . . . . . . . . . . . . . . 41

2.7 Pastilhas em Cisalhamento . . . . . . . . . . . . . . . . 41

2.8 Conclusão . . . . . . . . . . . . . . . . . . . . . . 46

3 Acoplamento dos circuitos elétricos nas estruturas piezoelétricas 47

3.1 Relação entre cargas elétricas nos circuitos e nos elementos piezoelétricos 48

3.2 Equações de movimento para o sistema acoplado . . . . . . . . . . . . . . . 49

3.2.1 Equações do movimento . . . . . . . . . . . . . . . . 50

3.3 Análise Harmônica . . . . . . . . . . . . . . . . . . . . . 51

3.3.1 Excitação puramente mecânica . . . . . . . . . . . . . 51

3.3.2 Excitação puramente elétrica . . . . . . . . . . . . . . . . 52

3.4 Projeto e análise de controle passivo de vibrações . . . . . . . . . . . . . . . . 54

3.5 Projeto do absorvedor dinâmico de vibrações . . . . . . . . . . . . . 55 
3.6 Projeto da lei de controle . . . . . . . . . . . . . . . . . . . 62

3.7 Resultados para o controle ativo-passivo . . . . . . . . . . . . . . . 64

3.7.1 Estrutura com cerâmica piezoelétrica em extensão . . . . . . . . 64

4 Modelagem estocástica para análise de incertezas $\quad 67$

4.1 Análise dos resultados obtidos . . . . . . . . . . . . . . . 70

4.1.1 Análise da influência de incertezas para propriedades dielétricas do material $\left(\bar{\beta}_{33}^{\varepsilon}\right) \ldots \ldots \ldots \ldots$. . . . . . . . . . . 70

4.1.2 Análise da influência de incertezas para propriedades piezelétricas do material $\left(h_{31}\right) \quad \ldots \ldots \ldots \ldots \ldots$

4.1.3 Análise da influência de incertezas para as propriedades resistivas do circuito elétrico $R_{c} \ldots \ldots \ldots \ldots \ldots \ldots 72$

4.1.4 Análise da influência de incertezas para propriedades indutivas do circuito elétrico $L_{c} \ldots \ldots \ldots \ldots \ldots$

4.1.5 Análise da influência das incertezas para todas as variáveis consideradas ........................ 75

5 Otimização da resistência e indutância em função da tensão aplicada $\quad 79$

5.1 Definição do problema a ser otimizado . . . . . . . . . . . . . . . 79

5.2 Metodologia de otimização dos parâmetros de circuito elétrico utilizados para o APPN . . . . . . . . . . . . . . . . . 81

5.3 Resultados da otimização para estrutura tipo placa com L de $500 \mathrm{~mm}$. . . 83

5.4 Resposta em frequência para estruturas com R e L otimizados . . . . . . 87

6 Análise estocástica para incertezas na interface pastilha/placa (cola) 93

6.1 Estudo de diferentes funções de probabilidade . . . . . . . . . . . . . . . 93

6.1.1 Função densidade de probabilidade Gamma . . . . . . . . . . . . 94

6.1.2 Função densidade de probabilidade Beta . . . . . . . . . . . . 96

6.1.3 Função densidade de probabilidade Gaussiana . . . . . . . . . . . 97

6.2 Análise dos resultados obtidos para estrutura tipo placa 500 x 400 com cola 99

6.3 Comparação . . . . . . . . . . . . . . . . 105

$\begin{array}{lll}7 & \text { Conclusões } & 107\end{array}$ 


\section{Lista de Figuras}

2.1 Deslocamentos de uma placa sanduíche considerando as hipóteses de Bernoulli-Euler para as camadas superior e inferior e Timoshenko para a camada central. . . . . . . . . . . . . . . .

2.2 Configuração de elemento finito com 4 nós utilizado na elaboração do modelo. . . . . . . . . . . . . . . . 30

2.3 Configuração para análise de piezo em extensão . . . . . . . . . . . . . . 41

2.4 Placa sanduíche simplesmente apoiada com camada piezelétrica em cisalhamento . . . . . . . . . . . . . . . . 43

2.5 Viga sanduíche "infinita"biapoiada com camada piezelétrica em cisalhamento . . . . . . . . . . . . . . . . . .

3.1 Configuração de um circuito elétrico com resistência (R), indutor (L), fonte de tensão $(\mathrm{V})$ e elemento piezoelétrico . . . . . . . . . . . . . . . . 47

3.2 Configuração da estrutura piezoelétrica em extensão . . . . . . . . . . . . 65

3.3 Primeira ressonância da viga com piezocerâmica em extensão: circuito aberto (traço longo ponto-pequeno); curto-circuito (traço longo ponto); circuito RL (traço curto ponto); circuito RLV (traço continuo) . . . . . . 66

4.1 Função de densidade para distribuição Gamma e histogramas das propriedades do material a) $\bar{\beta}_{33}^{\varepsilon}$, b) $\bar{h}_{31}$ e para parâmetros do circuito c) $R_{c}$ e d) $L_{c}$.

4.2 Convergencia das simulações realizadas através do método de Monte Carlos considerando incerteza nos parametros: (a) somente $L_{c}$ e (b) $\overline{\beta_{33}}, \overline{h_{31}}$, $h_{32}, R_{c}$ e $L_{c} \ldots \ldots \ldots \ldots \ldots$. . . . . . . . . . . . . .

4.3 Esquema do procedimento computacional para obtenção das respostas em frequencia e o calculo do intervalo de confiança.

4.4 Análise estocástica para o primeiro modo com incerteza na propriedade do material $\bar{\beta}_{33}^{\varepsilon}$ (a) Passivo Puro, (b) Ativo-Passivo com ganho constante e (c) Ativo-Passivo com ganho variável.

4.5 Análise estocástica para o primeiro modo com incerteza nas propriedades do material $h_{31}$ (a) Passivo Puro, (b) Ativo-Passivo com ganho constante e (c) Ativo-Passivo com ganho variável.

4.6 Análise estocástica para o primeiro modo com incerteza em $R_{c}$ no circuito elétrico (a) Passivo Puro, (b) Ativo-Passivo com ganho constante e (c) Ativo-Passivo com ganho variável. . . . . . . . . . . . . . . . . . . 74 
4.7 Análise estocástica para o primeiro modo com incerteza em $L_{c}$ no circuito elétrico (a) Passivo Puro, (b) Ativo-Passivo com ganho constante e (c) Ativo-Passivo com ganho variável. . . . . . . . . . . . . . . . 75

4.8 Análise estocástica para o primeiro modo com incerteza em todas as variáveis consideradas (a) Passivo Puro, (b) Ativo-Passivo com ganho constante e (c) Ativo-Passivo com ganho variável. . . . . . . . . . . . . .

5.1 Esquema de estruturas do tipo placa utilizadas para busca de uma nova estrutura para o processo de otimização com circuito elétrico conectado nas pastilhas piezelétricas. . . . . . . . . . . . . .

5.2 Esquema da estrutura tipo placa escolhida utilizada para otimização com circuito elétrico conectado nas pastilhas piezelétricas.

5.3 Variação da (a) resistência e (b) indutância em função da tensão aplicada para placa de $500 \mathrm{~mm}$ x $400 \mathrm{~mm}$ com $F_{1}(+$ azul $) ; F_{2}\left(*\right.$ vermelho) e $F_{3}\left(*_{-}\right.$

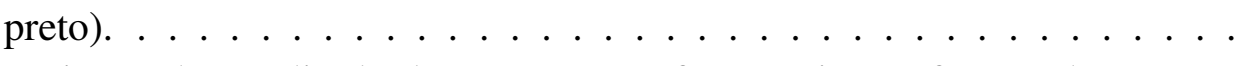

5.4 Variação da amplitude da resposta em frequência em função da tensão aplicada para placa de $500 \mathrm{~mm} \times 400 \mathrm{~mm}$ com diferentes $F_{1}(+$ azul $) ; F_{2}(*$ vermelho) e $F_{3}(*$ - preto $) \ldots \ldots \ldots \ldots \ldots \ldots$

5.5 Resposta da estrutura nos casos: circuito aberto (azul), passivo ótimo (verde), passivo (vermelho) e ativo-passivo (amarelo) com tensão de $V_{\max }=100 \mathrm{~V} \ldots \ldots \ldots \ldots \ldots \ldots \ldots \ldots$

5.6 Autoridade de controle para valores de resistência e indutância otimizados para os casos puramente V (verde) e RLV com $V_{\max }=100 \mathrm{~V}$ (amarelo).

5.7 Resposta da estrutura nos casos: circuito aberto (azul), passivo ótimo (verde), passivo (vermelho) e ativo-passivo (amarelo) com tensão de $V_{\max }=150 \mathrm{~V} \ldots \ldots \ldots \ldots \ldots \ldots \ldots \ldots$

5.8 Autoridade de controle para valores de resistência e indutância otimizados para os casos puramente passivo (verde) e ativo-passivo com $V_{\max }=$ $150 V$ (amarelo). . . . . . . . . . . . . . . .

5.9 Resposta em frequência da estrutura em circuito aberto (continua azul) e com controle ativo-passivo (- -) para diferentes valores de tensão máxima no intervalo de 10 a $250 \mathrm{~V}$ e para diferentes amplitudes de força de perturbação $F_{1}\left(\right.$ a),$F_{2}\left(\right.$ b) e $F_{3}($ c $) \ldots \ldots \ldots \ldots$

6.1 Histograma com os valores utilizados para o modelo estocástico através de uma Gamma . . . . . . . . . . . . . . . . . . . . .

6.2 Esquema do procedimento computacional para obtenção das respostas em frequência e o calculo do intervalo de confiança para a função densidade de probabilidade Gamma.

6.3 Histograma com os valores utilizados para o modelo estocástico através de função densidade de probabilidade Beta . . . . . . . . . . . . .

6.4 Histograma com os valores utilizados para o modelo estocástico através de uma função gaussiana . . . . . . . . . . . . . . . . .

6.5 Histograma com os valores utilizados para o modelo estocástico através de uma função densidade de probabilidade gaussiana truncada . . . . . 100 
6.6 Esquema da estrutura tipo placa utilizada para análise do efeito da cola entre os elementos piezelétricos e estrutura, com circuito elétrico conectado nas pastilhas piezelétricas. . . . . . . . . . . . . . . . 101

6.7 Intervalo de confiança para análise estocástica para o primeiro modo com incerteza na cola, acoplado a um circuito elétrico controle puramente passivo. . . . . . . . . . . . . . . . . 102

6.8 Intervalo de confiança para análise estocástica para o primeiro modo com incerteza na cola acoplado a um circuito elétrico controle puramente passivo $[$ zoom $] \ldots \ldots \ldots$. . . . . . . . . . . . . . . . . . 102

6.9 Intervalo de confiança para análise estocástica para o primeiro modo com incerteza na cola acoplado a um circuito elétrico controle ativo-passivo com ganho constante. . . . . . . . . . . . . . . . . . . . 103

6.10 Intervalo de confiança para análise estocástica para o primeiro modo com incerteza na cola acoplado a um circuito elétrico controle ativo-passivo com ganho variável [zoom].

6.11 Intervalo de confiança para análise estocástica para o primeiro modo com incerteza na cola acoplado a um circuito elétrico controle ativo-passivo com ganho variável. . . . . . . . . . . . . . . . . . . .

6.12 Intervalo de confiança para análise estocástica para o primeiro modo com incerteza na cola acoplado a um circuito elétrico controle ativo-passivo com ganho variável [zoom].

6.13 Comparação das diferentes respostas em frequência para o primeiro modo de vibração na mesma estrutura utilizando a distribuição Gamma (a), distribuição Beta (b) e distribuição Gaussiana truncada. . . . . . . . . . . 106 


\section{Capítulo 1}

\section{Introdução}

\subsection{Introdução}

Vibrações estruturais geralmente são indesejáveis pois podem causar problemas como fadiga estrutural, transmissão de vibrações para outros sistemas acoplados, ruído externo ou interno, entre outros problemas que acabam afetando o funcionamento ou rendimento de máquinas e estruturas. Estudos mostram que é possível limitar ou minimizar os efeitos destas vibrações através de técnicas de atenuação de vibrações.

Técnicas de atenuação consistem em diminuir as amplitudes das vibrações estruturais nas regiões próximas às frequências de ressonância. Pode-se dividir estas técnicas em três grupos distintos: passivos, ativos e ativo-passivos. As técnicas passivas de atenuação de vibrações estruturais consistem na integração ou adição de materiais e/ou sistemas à estrutura original fazendo com que parte de suas vibrações possam ser absorvidas e, então, dissipadas ou armazenadas.

Pode-se também controlar vibrações estruturais utilizando técnicas ativas que incorporam atuadores em pontos específicos na estrutura. Estes atuadores aplicam forças na estrutura de acordo com uma lei de controle para minimizar a vibração original.

A necessidade de um sistema de controle que ao mesmo tempo seja seguro e robusto, como o controle passivo, e eficiente e ajustável, como o controle ativo, incentivou 
o desenvolvimento de sistemas de controle híbridos ativo-passivos, combinando os tratamentos passivos e ativos em um único e singular tipo de controle. Este tipo de controle híbrido ativo-passivo poderia assegurar segurança e robustez ao sistema no caso de mal funcionamento do controle ativo e ainda melhorar o desempenho do sistema para algumas frequências selecionadas em comparação com o controle passivo.

Um grupo de materiais que tem sido bastante utilizados para o controle de vibrações através de técnicas passivas e/ou ativas são os materiais piezelétricos. Estes materiais tem a capacidade de transformar a energia mecânica proveniente das vibrações em energia elétrica ou quando excitados com um sinal elétrico produzem tensões mecânicas que são transmitidas para a estrutura e assim atuam sobre ela para o controle ativo de suas vibrações.

\subsection{Revisão Bibliográfica}

Nas próximas seções, uma revisão da literatura relacionada as diferentes áreas de pesquisa relevantes para a realização deste trabalho é apresentada com o enfoque nos aspectos: $i$ ) controle passivo de vibrações usando materiais piezelétricos conectados a circuitos shunt, $i$ ) controle ativo de vibrações usando sensores e atuadores piezelétricos, iii) controle híbrido ativo-passivo usando sensores/atuadores piezelétricos conectados a circuitos ativo-passivos e $i v$ ) análise de incertezas.

\subsubsection{Materiais piezelétricos e seus modos de atuação}

Piezeletricidade é uma propriedade que um grupo seleto de materiais possui e foi descoberta em 1880 por Jacques e Pierre Curie durante o estudo sistemático do efeito da pressão na geração de corrente elétrica em cristais, como o quartzo, misturas de zircônia e turmalina. A expressão é derivada do grego indicando pressionar, consequentemente, piezoeletricidade é a geração de eletricidade através de uma pressão mecânica. 
Na piezeletricidade, a magnitude da polarização é dependente da magnitude da tensão. Sem polarização, a cerâmica é inativa, com polarização entretanto, ela se torna funcional em várias aplicações. Um dos problemas das cerâmicas piezelétricas é a temperatura de Curie, onde a cerâmica perde sua polarização e conseqüentemente o seu efeito piezelétrico. Dois efeitos piezelétricos são de extrema importância, pois são os efeitos que são utilizados em técnicas de controles. O primeiro é o efeito direto (muitas vezes designado como gerador), este efeito é produzido através de uma tensão mecânica gerando uma corrente elétrica. O segundo é o efeito inverso, onde a aplicação de uma tensão elétrica nas superfícies da cerâmica piezelétrica gera uma tensão mecânica.

As equações básicas que descrevem estes dois efeitos levam em consideração as propriedades elétricas e elásticas do material;

$$
\mathcal{E}=\mathbf{s}^{E} \boldsymbol{\sigma}+\mathbf{d}^{\mathbf{t}} \mathbf{E}
$$

e

$$
\mathbf{D}=\mathbf{d} \boldsymbol{\sigma}+\boldsymbol{\epsilon}^{\sigma} \mathbf{E}
$$

onde $\mathbf{D}$ é o deslocamento elétrico, $\varepsilon$ é a tensor de deformações mecânicas, $\mathbf{E}$ o campo elétrico, $\boldsymbol{\sigma}$ tensor das tensões mecânicas, $\mathbf{d}$ o coeficiente piezelétrico, $\mathbf{s}$ é o tensor dos coeficientes de flexibilidade medido a campo elétrico constante e $\epsilon$ é o tensor dos coeficientes dielétricos medido a tensão constante [27, 29]. Essas equações podem ser muito simplificadas quando o elemento piezelétrico é carregado uniaxialmente com uma tensão normal ou cisalhante e só um par de eletrodos está presente para gerar um campo elétrico externo com componentes em uma só direção $[8,26]$.

Estes modos comuns de operação podem ser descritos como: caso longitudinal (deformação e campo na direção 3), caso transverso (deformação na direção 1 ou 2 e campo na direção 3), caso cisalhante (deformação na direção 4(23) ou 5(13) e campo na direção 2 ou 1 respectivamente). Quando se deseja obter um deslocamento transver- 
sal do material piezelétrico, pode-se também fazê-lo atuar por cisalhamento na estrutura. Isto pode ser obtido aplicando-se um campo elétrico perpendicular à polarização da pastilha. Assim, para uma pastilha polarizada na direção de sua espessura, o campo elétrico pode ser aplicado na direção do comprimento ou largura. Alternativamente, se a pastilha cerâmica estiver polarizada na direção de seu comprimento ou largura, basta que se aplique um campo na direção de sua espessura, ou seja, na direção perpendicular à polarização, que o material piezelétrico irá apresentar deformações de cisalhamento. Cada um desses modos está associado com um característico coeficiente de acoplamento eletromecânico que mede a taxa de conversão de energia mecânica em elétrica. Pelas características acima descritas, cerâmicas piezelétricas se tornaram peças importantes em aplicações de controle de vibrações.

As cerâmicas piezelétricas são materiais policristalinos, como o Titanato Zirconato de Chumbo, em geral denominado PZT, estes materiais são relativamente fáceis de produzir e mostram um expressivo coeficiente de acoplamento eletromecânico.

Conseqüentemente, estes materiais são amplamente utilizados para construção de transdutores piezelétricos, que são disponíveis em muitas formas. O mais comum é colar pastilhas finas de cerâmica, no modo de extensão, sobre superfícies de estruturas flexíveis de maneira que, quando usadas como atuadores (tensão elétrica aplicada aos eletrodos da pastilha), geram momentos fletores na estrutura e, quando usadas como sensores (tensão ou carga elétrica medida nos eletrodos da pastilha), permitem medir a deformação da estrutura [17] .

Embora grande parte dos estudos realizados até então consista em colar cerâmicas piezelétricas na superfície da estrutura a ser controlada ou monitorada por facilidade construtiva, o uso crescente de materiais compósitos laminados permite que se pense em projetar estruturas laminadas possuindo, além de suas camadas com objetivos estruturais, algumas camadas piezelétricas para o controle ou monitoramento contínuo da estrutura.

Para aplicação em estruturas laminadas, pastilhas piezelétricas polarizadas ao longo 
de seu comprimento ou largura (ao invés de ao longo da espessura como habitualmente) podem dar origem a um melhor acoplamento eletromecânico. Este novo conceito faz com que a pastilha piezelétrica atue usando o seu modo de cisalhamento.

Este conceito foi proposto inicialmente por Sun e Zhang [50] e estudos mostram que quando os atuadores piezelétricos estão dispostos desta maneira, induzem momentos distribuídos, ao contrário de forças concentradas no contorno como os atuadores em extensão, ou seja, estariam menos sujeitos a problemas de delimitação e teriam um desempenho menos dependente do tamanho e posição.

O mecanismo de atuação em cisalhamento também se mostra eficaz para atuadores com espessuras reduzidas e estruturas mais flexíveis. Diante de todos os pontos citados, pode-se afirmar que atuadores em cisalhamento podem ser interessantes para o controle de vibrações em comparação aos atuadores em extensão [26, 18, 10, 50].

\subsubsection{Modelagem de estruturas piezelétricas}

Diversos modelos têm sido propostos para prever o comportamento de estruturas laminadas com camadas de materiais piezelétricos atuando como atuadores, sensores ou meramente conversores de energia $[17,8,24]$. Um dos primeiros trabalhos nesta área foi publicado em 1970 por Allik e Hughes [3] o qual apresenta uma formulação variacional para o problema de vibração de uma estrutura com elementos piezelétricos usando o método dos elementos finitos com elementos tridimensionais com 12 graus de liberdade para deslocamentos mecânicos e 4 graus de liberdade elétricos.

Crawley [18] desenvolveu inúmeros estudos para problemas de vigas com sensores/atuadores piezelétricos utilizando uma formulação baseada na teoria de EulerBernoulli obtendo soluções exatas e numéricas.

Saravanos, Heyliger e Hopkins [43] propuseram uma formulação geral para a análise estática e dinâmica de placas laminadas considerando deslocamentos discretos para camada (teoria hoje denominada layerwise) dando origem a bons resultados embora 
com alto custo computacional.

Reddy [39] por outro lado, apresentou uma formulação de camada única equivalente (Equivalent Single Layer) associada a diferentes teorias de cisalhamento transverso para prover resultados satisfatórios para estruturas laminadas com custo computacional significativamente inferior. As tradicionais teorias CLPT (Classical Laminate Plate Theory ou Kirchoff-Love) e FSDT (First-Order Shear Deformation Theory) foram aplicadas a placas laminadas. Adicionalmente, um teoria de terceira ordem TSDT (Third-Order Shear Deformation Theory) foi proposta cuja principal vantagem seria não requerer fatores de correção de cisalhamento. As teorias foram aplicadas a placas laminadas com sensores e atuadores piezelétricos.

Benjeddou [8] examinou e discutiu os avanços e tendências de modelagem para estruturas adaptativas, principalmente aquelas com materiais piezelétricos por elementos finitos, destacando ainda tipos de análise que foram pouco exploradas na última década.

Gopinathan [24] apresentou uma revisão sobre teorias de placas laminadas, realizando uma análise dinâmica e mostrando um modelo de placa com FSDT, comparando-a à solução exata tridimensional.

Baillargeon e Vel [6] utilizaram um modelo tridimensional com teoria LayerWise para placas laminadas com condições de contorno simplesmente apoiada, obtendo as 12 primeiras frequências naturais em circuito aberto e fechado.

Khdeir e Aldraihem [30] apresentaram soluções analíticas para vibrações em placas laminadas com camadas piezelétricas usando teorias ESL CLPT, FSDT e TSDT para diferentes condições de contorno.

Deü e Benjeddou [19] utilizaram uma modelagem semelhante e os resultados foram comparados a um modelo de elementos finitos e uma análise paramétrica foi realizada para o estudo do acoplamento eletromecânico em relação a posição das pastilhas piezelétricas na estrutura. 


\subsubsection{Controle passivo de vibrações usando materiais piezelétricos}

Sunar e Rao [49] apresentaram uma revisão sobre o uso de materiais piezelétricos para sensoriamento e controle de estruturas flexíveis, mostrando que quando incorporados na estrutura estes materiais conseguem uma precisão no sensoriamento e grande adaptação em controle para estruturas inteligentes.

Moheimani [34] também apresentou uma revisão sobre o uso de materiais piezelétricos como sensores e atuadores em controle de vibrações em estruturas flexíveis. Este trabalho, no entanto, enfatiza que, em aplicações para controle de vibrações, quando ocorre a deformação da estrutura o elemento piezelétrico converte uma porção desta energia em energia elétrica que poderia ser dissipada através de um circuito resistivo.

Ahmadian e DeGuilio [2] apresentaram uma revisão sobre materiais piezelétricos e sua associação a técnicas de controle passivo, ativo e simultâneo ativo-passivo de vibrações estruturais.

Hagood e von Flotow [25] apresentaram um estudo sobre a possibilidade da dissipação da energia mecânica utilizando materiais piezelétricos acoplados a um circuito elétrico. Foram estudados dois casos: o caso puramente resistivo e o caso onde o circuito elétrico possuía uma resistência e uma indutância. Para o caso puramente resistivo, o comportamento apresenta uma dependência com a frequência, similar aos viscoelásticos, porém mais independente da temperatura. No caso do circuito elétrico com resistência e indutância, observou-se a introdução de uma ressonância elétrica que pode ser otimizada e ajustada com a ressonância de vibração da estrutura analogamente ao caso do absorvedor mecânico de vibração.

Benjeddou e Ranger [13] apresentaram um modelo teórico juntamente com um modelo de elementos finitos para cerâmicas piezelétricas no modo de cisalhamento para controle passivo de vibrações chamadas SSD (Shear-mode Shunted Damping) e para cerâmicas piezelétricas no modo de extensão chamadas de ESD (Extension-mode Shunted Damping). 
Trindade e Maio [63] mostraram que o modo de cisalhamento, nos materiais piezelétricos é mais interessante para altas frequências e pequenos deslocamentos na estrutura. Foi dada especial atenção a formulação do modelo, o acoplamento entre as componentes mecânicas e elétricas do sistema e uma otimização dos parâmetros geométricos, mecânicos e elétricos.

Viana e Steffen [65] apresentaram um estudo sobre o amortecimento passivo multimodal de estruturas, com circuitos em série e em paralelo com as cerâmicas piezelétricas conectadas a estrutura, onde cerâmicas piezelétricas são conectadas a estrutura e individualmente controlam modos distintos. Foi realizado também um ajuste nos valores dos componentes do circuito, bem como as alternativas encontradas para conseguir uma indutância capaz de atingir os valores calculados, onde o caminho encontrado foi a produção de indutâncias sintéticas.

Thornburgh e Chattopadhay [58] apresentaram uma modelagem considerando simultaneamente os graus de liberdade mecânicos e elétricos em estruturas compósitas possibilitando assim um entendimento maior dos fatores de amortecimento da estrutura devido ao tipo de controle incorporado, sensoriamento e consumo elétrico.

Lesieutre [32] apresentou diversos tipos de circuitos elétricos dissipativos que podem ser utilizados em controle passivo de vibrações, entre eles estão os resistivos, indutivos, capacitivos e comutados (switched). O circuito capacitivo poderia mudar a rigidez do elemento piezelétrico provendo assim um absorvedor mecânico ajustável. O circuito comutado poderia ser mais eficiente pois requer menores valores de indutância porém tem comportamento não-linear.

\subsubsection{Controle ativo de vibrações usando materiais piezelétricos}

Bailey e Hubbard [5] apresentaram um desenvolvimento e análise de um controle ativo em vigas utilizando um parâmetro de distribuição para o atuador e para a teoria de controle com voltagem aplicada controlada. 
Dosch e Inman [21] desenvolveram uma técnica para uma simples cerâmica piezelétrica servindo de sensor e atuador em um sistema fechado. A base teórica utilizada para o desenvolvimento do atuador foi desenvolvida em termos das equações constitutivas eletromecânicas para materiais piezelétricos. A proposta de desenvolvimento de uma formulação assim é para tratar o amortecimento ativo de vibrações em vigas. Foi utilizada uma cerâmica piezelétrica para distribuir um momento na estrutura e assim garantir uma controlabilidade. Utilizaram leis de controle feedback e conseguiram com que o primeiro modo fosse suprimido, fazendo uma alteração na posição da cerâmica piezelétrica conectada a estrutura. Foi mostrado que é possível controlar dois modos de vibração simultaneamente.

Trindade, Benjeddou e Ohayon [61] apresentaram uma análise paramétrica para vigas piezelétricas em extensão e em cisalhamento. O modelo em elementos finitos utilizado considerou para as camadas externas o modelo de Bernoulli-Euler e para a camada central foi considerado o modelo de Timoshenko. Uma lei de controle LQR foi usada para maximizar o amortecimento dos quatro primeiros modos naturais de vibração para vigas do tipo sanduíche e obteram como resultado que vigas em sanduíche para atuadores em cisalhamento podem ser mais efetivas que cerâmicas em extensão quando se deseja um controle ativo de vibração.

Trindade, Benjeddou e Ohayon [60] apresentaram uma análise dos algoritmos de controles para estruturas do tipo sanduíche. Foram analisados três tipos de controle ativo: LQR, LQG e Feedback. Uma análise paramétrica para controle ativo indica que o controle LQR conseguiu controlar modos selecionados, enquanto o controle Feedback é menos efetivo que um controle LQR. O controle LQG mostrou uma performance melhor perante ao controle LQR.

Raja, Prathap e Sinha [38] utilizaram as equações quase estáticas, para materiais piezelétricos, a construção de uma formulação em elementos finitos capaz de modelar dois tipos de piezoelasticidade para atuação em vigas sanduíche. Utilizando um controle 
baseado em LQR e IMSC estimou-se o amortecimento ativo em atuadores cerâmicos piezelétricos em extensão e em cisalhamento. A performance de cada tipo de atuador foi estudada através da resposta dinâmica no domínio modal. Como resultado, foi observado que atuadores em cisalhamento são mais efetivos para controle ativo que cerâmicas piezelétricas em extensão.

Trindade e Benjeddou [60] apresentaram uma análise numérica de controle ativo de vibrações usando simultaneamente cerâmicas piezelétricas em extensão e cisalhamento em vigas sanduíche, demonstrando que, quando utiliza-se simultaneamente os modos de extensão e cisalhamento das cerâmicas piezelétricas, obtêm-se boas respostas desde que seja considerado um efeito complementar na atuação mecânica.

\subsubsection{Controle ativo-passivo de vibrações usando materiais pie- zelétricos}

O conceito do controle ativo-passivo, o chamado APPN (Active-Passive Piezoelectric Network), consiste em combinar as técnicas de controle passivas e ativas. Esta configuração foi proposta com o propósito de garantir uma maior controlabilidade da estrutura. Esta configuração integra junto com o material piezelétrico, uma fonte de voltagem, uma resistência e uma indutância.

Com o objetivo de reduzir o potencial elétrico necessário para viabilizar o controle ativo de vibrações, Sirohi e Chopra [51] também propuseram acoplar um circuito ressonante (resistivo-indutivo) em série com a fonte de tensão. A limitação prática de valores elevados de indutância foi estudada sugerindo o uso de pseudo-indutores.

Em Tsai e Wang [55] foi apresentado um novo método de analisar o conceito do APPN, mostrou-se que o circuito shunt não somente pode promover um controle passivo como também promove um aumento na autoridade de controle quando ajustado corretamente. Os resultados mostram que a integração do APPN na estrutura é mais efetiva que os sistemas separados. 
Tang e Wang [52] analisaram a autoridade de controle para o casos de controle puramente passivo e puramente ativo comparando posteriormente com o controle híbrido APPN. A comparação foi feita de modo adimensional, considerando a importância do coeficiente de acoplamento eletromecânico. Um método para aumentar o acoplamento do coeficiente eletromecânico foi proposto onde uma capacitância negativa do circuito é introduzida.

Morgan e Wang [35] apresentaram uma aproximação combinando um circuito elétrico e ações ativas onde o sistema é sintetizado. O controle ativo foi separado em três partes onde na primeira parte é realizado uma correção no valor do indutor, após é considerada uma resistência negativa e por ultimo um aumento no acoplamento eletromecânico.

Em Tang e Wang [54] foi realizada uma revisão sobre desenvolvimentos em controle híbrido onde apresentou-se um algoritmo para estudar o controle de vibração e maximizar o amortecimento no controle ativo e no controle passivo simultaneamente, realizando simultaneamente uma otimização nos valores dos componentes do circuito elétrico.

Jiang, Tang e Wang [28], no interesse de ajustar os valores dos componentes do circuito elétrico utilizado no controle APPN, realizaram um estudo da influência da indutância nas ressonâncias da estrutura para identificação de danos estruturais usando transdutores piezelétricos. Foi desenvolvido um algoritmo de identificação de alta ordem, onde fundamentalmente se promove uma melhoria na identificação de danos com o uso de medidas de frequências.

\subsubsection{Quantificação de incertezas e modelagem estocástica}

Os mecanismos de processos randômicos tem atraído a atenção de cientistas e engenheiros nas ultimas décadas e uma análise matemática tem sido desenvolvida e descrita para possibilitar um entendimento destes processos naturais ou em dispositivos feitos pelo homem. Pode-se verificar estes efeitos quando se observa a interação do vento na superfície 
do oceano, na distribuição das forças em sismologia e em vibrações estruturais devido a fadigas dos materiais utilizados [31].

Desta maneira, o estudo das incertezas e seus processos provêem um entendimento dos efeitos e suas correlações na dinâmica vibracional, utilizando artifícios matemáticos podemos elaborar um tratamento das variáveis e investigar suas influências em algumas aplicações.

Estudando os modelos computacionais para análise de vibrações e níveis internos de ruído, Durand e Soize [22] consideraram a alta complexidade de modelos estruturais/acústicos quando se leva em conta pequenas variações destas propriedades. O trabalho propôs um modelo probabilistico de incerteza, implementado e adaptado computacionalmente.

Soize [48] apresentou uma extensão do trabalho de incertezas com modelos computacionais para sistemas dinâmicos e com validação experimental destes modelos. Apresentou uma metodologia para identificação e validação de incertezas complexas em sistemas dinâmicos utilizando dados experimentais.

Em Soize [47], um novo conceito para análise de incertezas aleatórias em sistemas dinâmicos foi proposto. Este conceito propõe uma modelagem em sistemas mecânicos, submetidos a um impulso e predizendo as respostas dinâmicas da estrutura. O modelo probabilístico desenvolvido baseou-se no principio da máxima entropia aproveitando todas as informações disponiveis para o problema.

Soize e Chebil [46] propuseram uma nova abordagem, chamada de modelo aleatório de incertezas não paramétrico, introduzido para modelagem de incertezas aleatórias em elastodinâmica em baixa frequência. Demonstrou um estudo estatístico dos autovalores aleatórios em matrizes aleatórias em comparação ao GOE (Gaussian Orthogonal Ensemble). Este método foi validado e foi demonstrado um esforço computacional menor em comparação ao método GOE.

Soize e Batou [45] apresentaram um estudo em dinâmicas estruturais não linea- 
res, onde foi utilizado um modelo de incertezas que foram introduzidas em um modelo computacional e comparadas com um sistema real, obtendo uma validação do modelo proposto.

Cataldo, Soize e Sampaio [16] realizaram a construção de um sistema dinâmico não linear estocástico para geração de voz sintética. O sistema dinâmico possui alta não linearidade e o sinal de saída gerado é muito sensitivo aos parâmetros do sistema. Utilizando um tratamento estatístico foi desenvolvido um modelo para determinar a sensibilidade dos parâmetros, utilizando o principio de máxima entropia.

Pandit, Singh e Sheikh [37] utilizaram uma análise estocástica para as propriedades de placas sanduíche para investigar a deflexão transversa. O modelo utilizado foi um modelo de alta ordem proposto anteriormente pelos autores. A performance do modelo estocástico para estruturas sanduíche foi comparada com a média e os valores de desvio padrão de deflexão obtidas através de processos de Monte Carlo e comparados com a literatura.

Sett, Jeremíc e Kavvas [44] construíram um modelo de uma estrutura de material elástico com incertezas estocástica nas propriedades do material. Estas incertezas foram modeladas através de campos aleatórios e foram introduzidas nas equações de movimento mecânica. Foram utilizadas para comparação algumas teorias como a equação FokkerPlanck-Kolmogorov e os polinômios de caos.

Ritto, Soize e Sampaio [40] utilizaram modelos probabilisticos para determinar a performance para modelos de perfuração de rochas. Esta interação foi representada por equações constitutivas não lineares, as incertezas usadas foram de modelos probabilisticos não paramétricos. 


\subsection{Objetivos}

Este trabalho tem como objetivo principal propor um modelo em elementos finitos e métodos para a análise do desempenho de técnicas de controle passivo, ativo e ativopassivo de vibrações de placas sanduíche (três camadas) usando materiais piezelétricos incluindo aspectos de otimização de propriedades geométricas, componentes do circuito elétrico e parâmetros de controle, e de análise do efeito de incertezas paramétricas das propriedades dos materiais e dos componentes do circuito elétrico no desempenho do sistema em termos de atenuação das vibrações estruturais. 


\section{Capítulo 2}

\section{Modelo em elementos finitos de placas}

\section{sanduíche piezelétricas}

\subsection{Hipóteses cinemáticas e equações constitutivas}

Apresenta-se neste capítulo uma formulação em elementos finitos para placas sanduíche piezelétricas. As camadas externas da placa possuem polarização na direção da espessura, enquanto que a camada central possui polarização na direção longitudinal. A hipótese de Bernoulli-Euler é utilizada nas camadas externas enquanto que na camada central, usa-se as hipóteses de Timoshenko. Considera-se também as hipóteses de tensões planas para todas as camadas. $\mathrm{O}$ acoplamento da estrutura mecânica com a parte elétrica constituída por um ou $n$ circuitos elétricos é levado em conta no conjunto de equações. Eletrodos cobrem totalmente as camadas piezelétricas nas superfícies superiores e inferiores das camadas piezelétricas garantindo assim um estado equipotencial elétrico em toda sua extensão. Por simplicidade, supõe-se que todas as camadas são compostas por materiais piezelétricos ortotrópicos e perfeitamente colados de modo a garantir a continuidade de deslocamentos nas interfaces entre as camadas. 


\subsubsection{Deslocamentos e deformações}

Os deslocamentos das camadas, superior $(t)$ e inferior $(b)$, podem ser escritos de forma a garantir que os deslocamentos independentes de cada uma das camadas e a garantir que nas interfaces de contato entre as camadas superior e inferior com a camada central $((c))$ tenha-se o mesmo deslocamento no ponto de contato. Os deslocamentos para as camadas externas podem ser descritos como,

$$
\begin{aligned}
& \bar{u}_{i}(x, y, z)=u_{i}(x, y)+\left(z-z_{i}\right) \beta_{i}(x, y) ; \quad i=t, b \\
& \bar{v}_{i}(x, y, z)=v_{i}(x, y)+\left(z-z_{i}\right) \gamma_{i}(x, y) \\
& \bar{w}_{i}(x, y, z)=w(x, y)
\end{aligned}
$$

sendo que $u_{i}$ e $v_{i}$ são os deslocamentos axiais dos planos médios da $i$-ésima camada, $w_{i}$ é o deslocamento transversal (deflexões) e $\beta_{i}$ e $\gamma_{i}$ são os ângulos de rotação das seções transversais, onde podemos escrever $\beta_{i}=-\frac{\partial w}{\partial x}$ e $\gamma_{i}=-\frac{\partial w}{\partial y}$.

Para a camada central, denominada como central $(c)$, considerando as hipóteses de Timoshenko,

$$
\begin{aligned}
& \bar{u}_{c}(x, y, z)=u_{c}(x, y)+\left(z-z_{c}\right) \phi_{x}(x, y) \\
& \bar{v}_{c}(x, y, z)=v_{c}(x, y)+\left(z-z_{c}\right) \phi_{y}(x, y) \\
& \bar{w}_{c}(x, y, z)=w(x, y)
\end{aligned}
$$

$z_{i}$ é a posição do plano médio da $i$-ésima camada na direção $z$ e $z_{c}=0$. Utilizando-se da continuidade de deslocamento entre as camadas, ou seja, as camadas estão perfeitamente coladas fazendo com que o deslocamento no ponto de contato seja igual para ambas as camadas, podemos escrever os deslocamentos da camada central somente em função dos deslocamentos das camadas externas, $u_{t}, u_{b}$ e $w$, tal que

$$
u_{t}-\frac{h_{t}}{2} \frac{\partial w}{\partial x}=u_{c}+\frac{h_{c}}{2} \phi_{x}
$$




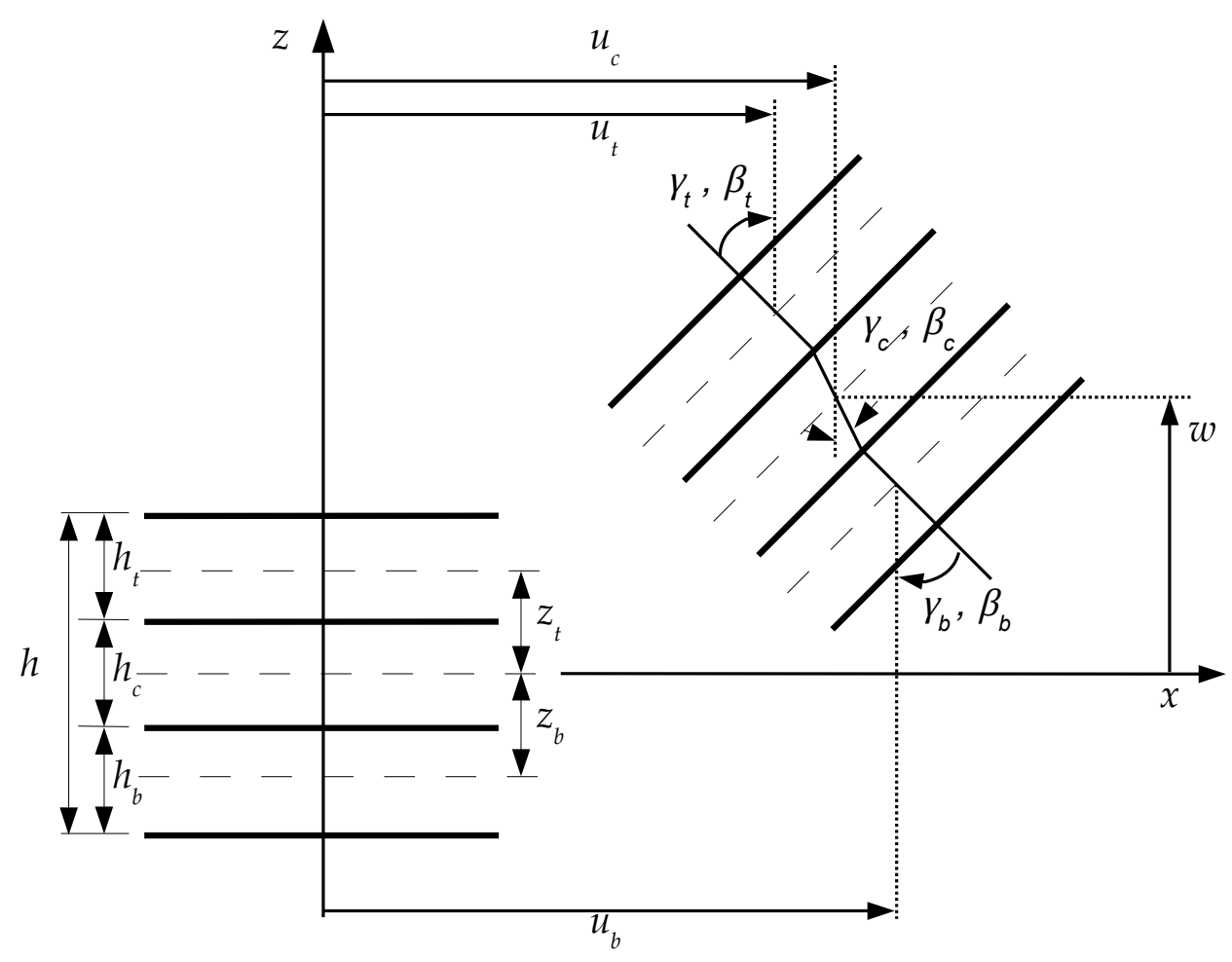

Figura 2.1: Deslocamentos de uma placa sanduíche considerando as hipóteses de Bernoulli-Euler para as camadas superior e inferior e Timoshenko para a camada central.

$\mathrm{e}$

$$
u_{c}-\frac{h_{c}}{2} \phi_{x}=u_{b}+\frac{h_{b}}{2} \frac{\partial w}{\partial x}
$$

Assim podemos escrever $u_{c}$ e $\phi_{x}$ como

$$
u_{c}=\frac{u_{t}+u_{b}}{2}-\frac{h_{d}}{4} \frac{\partial w}{\partial x} \quad \text { e } \quad \phi_{x}=\frac{u_{t}-u_{b}}{h_{c}}-\frac{h_{m}}{h_{c}} \frac{\partial w}{\partial x}
$$

Da mesma maneira que foi realizado para a direção x, podemos escrever os deslocamentos para a camada central em função dos deslocamentos das camadas externas para a direção y, 


$$
v_{t}-\frac{h_{t}}{2} \frac{\partial w}{\partial y}=v_{c}+\frac{h_{c}}{2} \phi_{y}
$$

$\mathrm{e}$

$$
v_{c}-\frac{h_{c}}{2} \phi_{y}=v_{b}+\frac{h_{b}}{2} \frac{\partial w}{\partial y}
$$

Assim podemos escrever $v_{c}$ e $\phi_{y}$ como

$$
v_{c}=\frac{v_{t}+v_{b}}{2}-\frac{h_{d}}{4} \frac{\partial w}{\partial y} \quad \text { e } \quad \phi_{y}=\frac{v_{t}-v_{b}}{h_{c}}-\frac{h_{m}}{h_{c}} \frac{\partial w}{\partial y}
$$

onde $h_{m}$ e $h_{d}$ são, respectivamente, a média aritmética e a diferença das espessuras das camadas superior e inferior, $h_{t}$ e $h_{b}$,

$$
h_{m}=\frac{h_{t}+h_{b}}{2} \quad \text { e } \quad h_{d}=h_{t}-h_{b}
$$

As relações para as deformações nas camadas superior e inferior são definidas como,

$$
\begin{aligned}
\mathcal{E}_{1 i} & =\frac{\partial \bar{u}_{i}}{\partial x}=\varepsilon_{1 m i}+\left(z-z_{i}\right) \varepsilon_{1 f i} \\
\mathcal{E}_{2 i} & =\frac{\partial \bar{v}_{i}}{\partial y}=\varepsilon_{2 m i}+\left(z-z_{i}\right) \varepsilon_{2 f i} \\
\varepsilon_{3 i} & =\frac{\partial \bar{w}}{\partial z}=0 \\
\mathcal{E}_{4 i} & =\frac{\partial \bar{v}_{i}}{\partial z}+\frac{\partial w_{i}}{\partial y}=0 \\
\mathcal{E}_{5 i} & =\frac{\partial \bar{u}_{i}}{\partial z}+\frac{\partial w_{i}}{\partial x}=0 \\
\overline{\mathcal{E}}_{6 i} & =\frac{\partial \bar{u}_{i}}{\partial y}+\frac{\partial \bar{v}_{i}}{\partial x}=\varepsilon_{6 i}+\left(z-z_{i}\right) \kappa_{6 i}
\end{aligned}
$$

onde as deformações de membrana e flexão, $\mathcal{E}_{m i}$ e $\varepsilon_{f i}$ respectivamente, podem ser escritas como sendo, 


$$
\begin{aligned}
& \mathcal{E}_{1 m i}=\frac{\partial u_{i}}{\partial x} \text { e } \varepsilon_{1 f i}=-\frac{\partial^{2} w}{\partial x^{2}} \\
& \varepsilon_{2 m i}=\frac{\partial v_{i}}{\partial y} \text { e } \varepsilon_{2 f i}=-\frac{\partial^{2} w}{\partial y^{2}} \\
& \varepsilon_{6 i}=\frac{\partial u_{i}}{\partial y}+\frac{\partial v_{i}}{\partial x} \text { e } \kappa_{6 i}=-2 \frac{\partial w}{\partial y \partial x}
\end{aligned}
$$

para as camadas superior e inferior, respectivamente $(i=t, b)$.

As relações usuais utilizadas em formulações FSDT (First-order Shear Deformation Theory) para as deformações na camada central são definidas como,

$$
\begin{aligned}
\varepsilon_{1 c} & =\frac{\partial \bar{u}_{c}}{\partial x}=\varepsilon_{1 m c}+z \varepsilon_{1 f c} \\
\varepsilon_{2 c} & =\frac{\partial \bar{v}_{c}}{\partial y}=\varepsilon_{2 m c}+z \varepsilon_{2 f c} \\
\varepsilon_{3 c} & =\frac{\partial \bar{w}}{\partial z}=0 \\
\mathcal{E}_{4 c} & =\frac{\partial \bar{v}_{c}}{\partial z}+\frac{\partial w_{c}}{\partial y} \\
\mathcal{E}_{5 c} & =\frac{\partial \bar{u}_{c}}{\partial z}+\frac{\partial w_{c}}{\partial x} \\
\bar{\varepsilon}_{6 c} & =\frac{\partial \bar{u}_{c}}{\partial y}+\frac{\partial v_{c}}{\partial x}=\varepsilon_{6 c}+z \kappa_{6 c}
\end{aligned}
$$

As deformações da camada central podem ser escritas como sendo,

$$
\begin{aligned}
& \varepsilon_{1 m c}=\frac{\partial u_{c}}{\partial x} \text { e } \varepsilon_{1 f c}=\frac{\partial \phi_{x}}{\partial x} \\
& \mathcal{E}_{2 m c}=\frac{\partial v_{c}}{\partial y} \text { e } \varepsilon_{2 f c}=\frac{\partial \phi_{y}}{\partial y} \\
& \varepsilon_{4 c}=\frac{\partial w}{\partial y}+\phi_{y} \\
& \varepsilon_{5 c}=\frac{\partial w}{\partial x}+\phi_{x} \\
& \mathcal{E}_{6 c}=\frac{\partial u_{c}}{\partial y}+\frac{\partial v_{c}}{\partial x} \text { e } \kappa_{6 c}=\frac{\partial \phi_{x}}{\partial y}+\frac{\partial \phi_{y}}{\partial x}
\end{aligned}
$$




\subsubsection{Equações constitutivas para materiais piezelétricos}

Como descrito anteriormente, materiais piezelétricos possuem um comportamento característico de acoplamento de tensões e deformações com o deslocamento elétrico gerado por suas propriedades internas. As equações constitutivas lineares que descrevem o comportamento destes materiais foram descritas por [27] e [29] onde podemos observar que,

$$
\left\{\begin{array}{l}
\boldsymbol{\sigma} \\
\mathbf{E}
\end{array}\right\}=\left[\begin{array}{cc}
\mathbf{c}^{D} & -\mathbf{h}^{t} \\
-\mathbf{h} & \boldsymbol{\beta}^{\mathcal{E}}
\end{array}\right]\left\{\begin{array}{l}
\mathcal{E} \\
\mathbf{D}
\end{array}\right\}
$$

onde $\mathbf{E}$ e $\mathbf{D}$ são os vetores de campo e deslocamento elétrico e $\boldsymbol{\sigma}$ e $\mathcal{E}$ são os vetores de tensão e deformação e o sobreescrito $t$ se refere à transposição da matriz. Materiais piezelétricos ortotrópicos com simetria axial paralela aos eixos principais da placa são considerados, para os quais $c_{i j}^{D}$, $h_{l j}$ e $\beta_{l}^{\varepsilon}(i, j=1, \ldots, 6 ; l=1,2,3)$ denotam as matrizes de constantes elásticas (para deslocamentos elétricos constantes), piezelétricas e dielétricas (para deformação constante), respectivamente. Para ambos os modos de extensão e cisalhamento das camadas que compõe a estrutura, somente o deslocamento elétrico transversal é considerado $\left(D_{1}=D_{2}=0\right)$ desde que as camadas da estrutura tenham eletrodos em suas faces a fim de garantir uma homogeneidade no deslocamento elétrico bem como no campo elétrico gerado pela deformação da estrutura. Como a camada central será considerada polarizada longitudinalmente enquanto que as camadas superior e inferior serão consideradas polarizadas na direção da espessura. As equações constitutivas tridimensionais (2.15) são reduzidas usando-se as hipóteses de deslocamento elétrico unidirecional $\left(D_{1}=D_{2}=0\right)$, tensões planas $\left(\sigma_{3}=0\right)$ e desconsiderando os campos que não contribuem para a energia do sistema. 


$$
\left\{\begin{array}{l}
\sigma_{1} \\
\sigma_{2} \\
\sigma_{3} \\
\sigma_{4} \\
\sigma_{5} \\
\sigma_{6} \\
\mathbf{E}_{1} \\
\mathbf{E}_{2} \\
\mathbf{E}_{3}
\end{array}\right\}=\left[\begin{array}{ccccccccc}
\mathbf{c}_{11}^{D} & \mathbf{c}_{12}^{D} & \mathbf{c}_{13}^{D} & 0 & 0 & 0 & 0 & 0 & -\mathbf{h}_{31} \\
\mathbf{c}_{12}^{D} & \mathbf{c}_{22}^{D} & \mathbf{c}_{23}^{D} & 0 & 0 & 0 & 0 & 0 & -\mathbf{h}_{32} \\
\mathbf{c}_{13}^{D} & \mathbf{c}_{23}^{D} & \mathbf{c}_{33}^{D} & 0 & 0 & 0 & 0 & 0 & -\mathbf{h}_{33} \\
0 & 0 & 0 & \mathbf{c}_{44}^{D} & 0 & 0 & 0 & -\mathbf{h}_{24} & 0 \\
0 & 0 & 0 & 0 & \mathbf{c}_{55}^{D} & 0 & -\mathbf{h}_{15} & 0 & 0 \\
0 & 0 & 0 & 0 & 0 & \mathbf{c}_{66}^{D} & 0 & 0 & 0 \\
0 & 0 & 0 & 0 & -\mathbf{h}_{15} & 0 & \beta_{11}^{\varepsilon} & 0 & 0 \\
0 & 0 & 0 & -\mathbf{h}_{24} & 0 & 0 & 0 & \beta_{22}^{\varepsilon} & 0 \\
-\mathbf{h}_{31} & -\mathbf{h}_{32} & -\mathbf{h}_{33} & 0 & 0 & 0 & 0 & 0 & \beta_{33}^{\varepsilon}
\end{array}\right]\left\{\begin{array}{c}
\varepsilon_{1} \\
\varepsilon_{2} \\
\varepsilon_{3} \\
\varepsilon_{4} \\
\varepsilon_{5} \\
\varepsilon_{6} \\
\mathbf{D}_{1} \\
\mathbf{D}_{2} \\
\mathbf{D}_{3}
\end{array}\right\}
$$

Simplificando as equações constitutivas para as camadas com polarização no seu sentido transversal, polarização em z $(\mathrm{PE})$, podemos escrever,

$$
\left[\begin{array}{c}
\sigma_{1} \\
\sigma_{2} \\
\sigma_{4} \\
\sigma_{5} \\
\sigma_{6} \\
\mathbf{E}_{3}
\end{array}\right]=\left[\begin{array}{cccccc}
\overline{\mathbf{c}}_{11}^{D} & \overline{\mathbf{c}}_{12}^{D} & 0 & 0 & 0 & -\overline{\mathbf{h}}_{31} \\
\overline{\mathbf{c}}_{12}^{D} & \overline{\mathbf{c}}_{22}^{D} & 0 & 0 & 0 & -\overline{\mathbf{h}}_{32} \\
0 & 0 & \overline{\mathbf{c}}_{44}^{D} & 0 & 0 & 0 \\
0 & 0 & 0 & \overline{\mathbf{c}}_{55}^{D} & 0 & 0 \\
0 & 0 & 0 & 0 & \overline{\mathbf{c}}_{66}^{D} & 0 \\
-\overline{\mathbf{h}}_{31} & -\overline{\mathbf{h}}_{32} & 0 & 0 & 0 & \overline{\boldsymbol{\beta}}_{33}^{\varepsilon}
\end{array}\right]\left[\begin{array}{c}
\mathcal{\varepsilon}_{1} \\
\mathcal{\varepsilon}_{2} \\
\mathcal{\varepsilon}_{4} \\
\mathcal{\varepsilon}_{5} \\
\mathcal{\varepsilon}_{6} \\
\mathbf{D}_{3}
\end{array}\right]
$$

Para as camadas com polarização no seu sentido horizontal, polarização em $x$ (PS), podemos escrever, 
Tabela 2.1: Propriedades dos materiais piezelétricos em função da polarização

\begin{tabular}{|l|c|c|}
\hline & Polarização (PS) $\rightarrow$ & Polarização (PE) $\uparrow$ \\
\hline $\bar{c}_{11}^{D}$ & $\left(c_{33}^{D}-\frac{c_{13}^{D}{ }^{2}}{c_{11}^{D}}\right)$ & $\left(c_{11}^{D}-\frac{c_{13}^{D} c^{D}}{c^{2}}\right)$ \\
\hline $\bar{c}_{12}^{D}$ & $\left(c_{23}^{D}-\frac{c_{12}^{D} c_{13}^{D}}{c_{11}^{D}}\right)$ & $\left(c_{12}^{D}-\frac{c_{13}^{D} c_{23}^{D}}{c_{33}^{D}}\right)$ \\
\hline $\bar{c}_{22}^{D}$ & $\left(c_{22}^{D}-\frac{c_{12}^{D}}{c_{11}^{D}}\right)$ & $\left(c_{22}^{D}-\frac{c_{23}^{D}}{c_{33}^{D}}\right)$ \\
\hline $\bar{c}_{44}^{D}$ & $c_{66}^{D}$ & $c_{44}^{D}$ \\
\hline $\bar{c}_{55}^{D}$ & $c_{55}^{D}$ & $c_{55}^{D}$ \\
\hline $\bar{c}_{66}^{D}$ & $c_{44}^{D}$ & $c_{66}^{D}$ \\
\hline $\bar{h}_{11}^{D}$ & $\left(h_{33}-\frac{h_{31} c_{13}^{D}}{c_{11}^{D}}\right)$ & 0 \\
\hline $\bar{h}_{12}^{D}$ & $\left(h_{32}-\frac{h_{31} c_{12}^{D}}{c_{11}^{D}}\right)$ & 0 \\
\hline $\bar{h}_{15}^{D}$ & 0 & $h_{15}$ \\
\hline $\bar{h}_{24}^{D}$ & 0 & $h_{24}$ \\
\hline $\bar{h}_{26}^{D}$ & $h_{24}$ & 0 \\
\hline $\bar{h}_{31}^{D}$ & 0 & $\left(h_{31}-\frac{h_{33} c_{13}^{D}}{c_{33}^{D}}\right)$ \\
\hline $\bar{h}_{32}^{D}$ & 0 & $\left(h_{32}-\frac{h_{33} c_{23}^{D}}{c_{33}^{D}}\right)$ \\
\hline $\bar{h}_{35}^{D}$ & $h_{15}$ & 0 \\
\hline $\bar{\beta}_{33}^{\varepsilon}$ & $\left.\beta_{11}^{\varepsilon}-\frac{h_{23}^{2}}{c_{33}^{D}}\right)$ \\
\hline
\end{tabular}

$$
\left[\begin{array}{c}
\sigma_{1} \\
\sigma_{2} \\
\sigma_{4} \\
\sigma_{5} \\
\sigma_{6} \\
\mathbf{E}_{3}
\end{array}\right]=\left[\begin{array}{cccccc}
\overline{\mathbf{c}}_{11}^{D} & \overline{\mathbf{c}}_{12}^{D} & 0 & 0 & 0 & 0 \\
\overline{\mathbf{c}}_{12}^{D} & \overline{\mathbf{c}}_{22}^{D} & 0 & 0 & 0 & 0 \\
0 & 0 & \overline{\mathbf{c}}_{44}^{D} & 0 & 0 & 0 \\
0 & 0 & 0 & \overline{\mathbf{c}}_{55}^{D} & 0 & -\overline{\mathbf{h}}_{35} \\
0 & 0 & 0 & 0 & \overline{\mathbf{c}}_{66}^{D} & 0 \\
0 & 0 & 0 & -\overline{\mathbf{h}}_{35} & 0 & \overline{\boldsymbol{\beta}}_{31}^{\varepsilon}
\end{array}\right]\left[\begin{array}{c}
\mathcal{E}_{1} \\
\mathcal{E}_{2} \\
\mathcal{E}_{4} \\
\mathcal{E}_{5} \\
\mathcal{E}_{6} \\
\mathbf{D}_{3}
\end{array}\right]
$$

Com as hipóteses assumidas, necessita-se realizar uma correção das propriedades dos materiais, dependente da sua polarização. Desta forma podemos construir a tabela 2.1. 


\subsection{Formulação variacional}

As equações de movimento podem ser escritas usando o seguinte princípio variacional estendido para cerâmicas piezelétricas

$$
\delta W^{c}-\delta W^{i n}+\delta W^{e x}=0
$$

onde $\delta W^{c}, \delta W^{i n}$ e $\delta W^{e x}$ são os trabalhos virtuais realizados pelas forças de inércia, internas e externas, respectivamente. Cada um destes trabalhos é composto de contribuições das três camadas piezelétricas que compõem a estrutura e serão detalhadas nas próximas seções.

\section{Trabalho virtual realizado pelas forças de inércia}

O trabalho virtual realizado pelas forças de inércia para a $i$-ésima camada da placa sanduíche pode ser escrito como

$$
\delta W_{i}^{c}=-\int_{V}\left(\delta \bar{u}_{i}^{t} \rho_{i} \ddot{\bar{u}}_{i}+\delta \bar{v}_{i}^{t} \rho_{i} \ddot{\bar{v}}_{i}+\delta \bar{w}_{i}^{t} \rho_{i} \ddot{\bar{w}}_{i}\right) d V_{i}
$$

onde $\rho_{i}$ é a densidade do material utilizado na $i$-ésima camada, os pontos denotam a derivação no tempo e $V_{i}$ é o volume da camada. Usando os campos de deslocamento definidos por (2.1), esta expressão pode ser reescrita como

$$
\begin{array}{r}
\delta W_{i}^{c}=-\int_{V}\left[\rho_{i}\left(\delta u_{i} \ddot{u}_{i}+\delta v_{i} \ddot{v}_{i}+\delta w \ddot{w}\right)-\left(z-z_{i}\right)\right. \\
\left(\delta u_{i} \ddot{w}_{x}+\delta w_{x} \ddot{u}_{i}+\delta v_{i} \ddot{w}_{y}+\delta w_{y} \ddot{v}_{i}\right) \\
\left.+\left(z-z_{i}\right)^{2}\left(\delta w_{x} \ddot{w}_{x}+\delta w_{y} \ddot{w}_{y}\right)\right] d V
\end{array}
$$

Supondo que todas as camadas são simétricas com relação às suas linhas neutras, $z=z_{i}$, a integração em $z$ resulta em 


$$
\delta W_{i}^{c}=-\int_{x} \int_{y}\left[I_{0 i}\left(\delta u_{i} \ddot{u}_{i}+\delta v_{i} \ddot{v}_{i}+\delta w \ddot{w}\right)+I_{2 i}\left(\delta w_{x} \ddot{w}_{x}+\delta w_{y} \ddot{w}_{y}\right)\right] d x d y
$$

sendo que $I_{0 i}$ e $I_{2 i}$ são os termos constantes definidos por,

$$
I_{0 i}=\int_{\frac{-h_{i}}{2}}^{\frac{h_{i}}{2}} \rho d z \quad \text { e } \quad I_{2 i}=\int_{z_{i}-\frac{h_{i}}{2}}^{z_{i}+\frac{h_{i}}{2}}\left(z-z_{i}\right)^{2} \rho d z
$$

lembrando que $i=t, b$ e $c$, representando a camada superior, inferior e central.

Para a camada central devemos lembrar que os deslocamentos, estão escritos em função das camadas superior e inferior e resultam na equação,

$$
\delta W_{i}^{c}=-\int_{x} \int_{y}\left[I_{0 c}\left(\delta u_{c} \ddot{u}_{c}+\delta v_{c} \ddot{v}_{c}+\delta w \ddot{w}\right)+I_{2 c}\left(\delta \phi_{x} \ddot{\phi}_{x}+\delta \phi_{y} \ddot{\phi}_{y}\right)\right] d x d y
$$

\section{Trabalho virtual realizado pelas forças internas eletromecânicas}

Para obter o trabalho virtual realizado pelas forças internas eletromecânicas, pode-se considerar a energia potencial (energia livre de Helmholtz) em uma camada piezelétrica

$$
H(\mathcal{\varepsilon}, \mathbf{D})=\frac{1}{2} \varepsilon^{t} \mathbf{c}^{D} \mathcal{E}-\varepsilon^{t} \mathbf{h} \mathbf{D}+\frac{1}{2} \mathbf{D}^{t} \boldsymbol{\beta}^{\varepsilon} \mathbf{D}
$$

escrita em termos de $\mathcal{\varepsilon}$ e $\mathbf{D}$, que são o vetor de deformações e o vetor de deslocamentos elétricos. $\mathbf{c}^{D}, \mathbf{h}$ e $\boldsymbol{\beta}^{\varepsilon}$ são as matrizes de constantes elásticas, piezelétricas e dielétricas. O trabalho virtual realizado pelas forças internas $\left(\delta W^{i n}\right)$ pode ser escrito como sendo o negativo da variação virtual da energia potencial $H$, que é composto de contribuições mecânica $\delta H_{m}$, eletromecânica (oriunda do efeito piezelétrico) $\delta H_{m e}$, e dielétrica $\delta H_{e}$ tal que $\delta W^{i n}=-\delta H=-\left(\delta H_{m}+\delta H_{m e}+\delta H_{e}\right)$.

Como citado anteriormente, um tratamento separado das camadas externas e central é necessário devido à diferente polarização do material que compõe estas camadas. 
Desta forma podemos escrever para as camadas externas,

$$
\delta H_{m i}=\int_{V}\left(\delta \varepsilon_{i}^{t} \overline{\mathbf{c}}_{i}^{D} \mathcal{\varepsilon}_{i}\right) d V
$$

considerando as expressões para as deformações desenvolvidas nas equações 2.11 e a Tabela 2.1 podemos obter,

$$
\delta H_{m i}=\int_{V}\left[\left(\delta \varepsilon_{1 i} \bar{c}_{11}^{D}+\delta \mathcal{E}_{2 i} \bar{c}_{12}^{D}\right) \varepsilon_{1 i}+\left(\delta \varepsilon_{1 i} \bar{c}_{12}^{D}+\delta \mathcal{E}_{2 i} \bar{c}_{22}^{D}\right) \varepsilon_{2 i}+\left(\delta \varepsilon_{6 i} \bar{c}_{66}^{D}\right) \varepsilon_{6 i}\right] d V
$$

Usando as expressões para as deformações (2.10), tem-se que

$$
\begin{aligned}
\delta H_{m i}=\int_{V}\left(\delta \varepsilon_{1 m i}+z \delta \varepsilon_{1 f i}\right) & \bar{c}_{11}^{D}\left(\varepsilon_{1 m i}+z \varepsilon_{1 f i}\right) \\
+ & \left(\delta \varepsilon_{2 m i}+z \delta \varepsilon_{2 f i}\right) \bar{c}_{12}^{D}\left(\varepsilon_{1 m i}+z \varepsilon_{1 f i}\right) \\
+ & \left(\delta \varepsilon_{1 m i}+z \delta \varepsilon_{1 f i}\right) \bar{c}_{12}^{D}\left(\varepsilon_{2 m i}+z \varepsilon_{2 f i}\right) \\
& +\left(\delta \varepsilon_{2 f i}+z \delta \varepsilon_{2 f i}\right) \bar{c}_{22}^{D}\left(\varepsilon_{2 f i}+z \varepsilon_{2 f i}\right) \\
& +\left(\delta \varepsilon_{6 i}+z \delta \kappa_{6 i}\right) \bar{c}_{66}^{D}\left(\varepsilon_{6 i}+z \kappa_{6 i}\right) d V
\end{aligned}
$$

distribuindo os termos temos,

$$
\begin{gathered}
\delta H_{m i}=\int_{V}\left[\left(\delta \varepsilon_{1 m i} \bar{c}_{11}^{D} \varepsilon_{1 m i}+\delta \varepsilon_{2 m i} \bar{c}_{12}^{D} \varepsilon_{1 m i}+\delta \varepsilon_{1 m i} \bar{c}_{12}^{D} \varepsilon_{2 m i}+\delta \varepsilon_{2 m i} \bar{c}_{22}^{D} \varepsilon_{2 m i}+\delta \varepsilon_{6}^{m} \bar{c}_{66}^{D} \varepsilon_{6}^{m}\right)\right. \\
+\left(z-z_{i}\right)\left(\delta \varepsilon_{1 f i} \bar{c}_{11}^{D} \varepsilon_{1 f i}+\delta \varepsilon_{1 f i} \bar{c}_{11}^{D} \varepsilon_{1 m i}+\delta \varepsilon_{2 m i} \bar{c}_{12}^{D} \varepsilon_{1 f i}+\delta \varepsilon_{2 f i} \bar{c}_{12}^{D} \varepsilon_{1 m i}+\right. \\
\delta \varepsilon_{1 m i} \bar{c}_{12}^{D} \varepsilon_{2 f i}+\delta \varepsilon_{1 f i} \bar{c}_{12}^{D} \varepsilon_{2 m i}+\delta \varepsilon_{2 f i} \bar{c}_{22}^{D} \varepsilon_{2 m i}+\delta \varepsilon_{2 m i} \bar{c}_{22}^{D} \varepsilon_{2 f i}+\delta \varepsilon_{6 i} \bar{c}_{66}^{D} \kappa_{6 i}+\delta \kappa_{6 i} \bar{c}_{66}^{D} \varepsilon_{6 i} \\
\left.+\left(z-z_{i}\right)^{2}\left(\delta \varepsilon_{1 f i} \bar{c}_{11}^{D} \varepsilon_{1 f i}+\delta \varepsilon_{2 f i} \bar{c}_{12}^{D} \varepsilon_{1 f i}+\delta \varepsilon_{1 f i} \bar{c}_{12}^{D} \varepsilon_{2 f i}+\delta \varepsilon_{2 f i} \bar{c}_{22}^{D} \varepsilon_{2 f i}+\delta \kappa_{6 i} \bar{c}_{66}^{D} \kappa_{6 i}\right)\right] d V
\end{gathered}
$$


Supondo novamente simetria nas camadas e integrando na seção transversal, obtemos,

$$
\begin{array}{r}
I_{3 i}=\int_{\frac{-h i}{2}}^{\frac{h i}{2}} d z \\
I_{4 i}=\int_{z_{i}-\frac{h i}{2}}^{z_{i}+\frac{h i}{2}}\left(z-z_{i}\right)^{2} d z
\end{array}
$$

e substituindo na equação acima, podemos simplificar o termo linear por simetria, a nova equação é escrita na forma,

$$
\begin{gathered}
\delta H_{m i}=\int_{x} \int_{y}\left[I _ { 3 i } \left(\delta \varepsilon_{1 m i} \bar{c}_{11}^{D} \varepsilon_{1 m i}+\delta \varepsilon_{2 m i} \bar{c}_{12}^{D} \varepsilon_{1 m i}+\delta \varepsilon_{1 m i} \bar{c}_{12}^{D} \varepsilon_{2 m i}+\right.\right. \\
\left.\delta \varepsilon_{2 m i} \bar{c}_{22}^{D} \varepsilon_{2 m i}+\delta \varepsilon_{6 i} \bar{c}_{66}^{D} \varepsilon_{6 i}\right) \\
+I_{4 i}\left(\delta \varepsilon_{1 f i} \bar{c}_{11}^{D} \varepsilon_{1 f i}+\delta \varepsilon_{2 f i} \bar{c}_{12}^{D} \varepsilon_{1 f i}+\delta \varepsilon_{1 f i} \bar{c}_{12}^{D} \varepsilon_{2 f i}+\right. \\
\left.\left.\delta \varepsilon_{2 f i} \bar{c}_{22}^{D} \varepsilon_{2 f i}+\delta \kappa_{6 i} \bar{c}_{66}^{D} \kappa_{6 i}\right)\right] d y d x
\end{gathered}
$$

Para a camada central, a energia de deformação para o cisalhamento também deve ser levada em consideração para o cálculo do trabalho virtual das forças internas, então

$$
\delta H_{m c}=\int_{V}\left(\delta \mathcal{E}^{t} \overline{\mathbf{c}}^{D} \mathcal{E}\right) d V
$$

considerando as expressões para as deformações desenvolvidas nas equações 2.13 , e a Tabela 2.1.2 podemos obter,

$$
\begin{aligned}
\delta H_{m c}=\int_{V}\left[\left(\delta \varepsilon_{1 c} \bar{c}_{11}^{D}+\delta \varepsilon_{2 c} \bar{c}_{12}^{D}\right)\right. & \varepsilon_{1 c}+ \\
& \left(\delta \varepsilon_{1 c} \bar{c}_{12}^{D}+\delta \varepsilon_{2 c} \bar{c}_{22}^{D}\right) \varepsilon_{2 c}+ \\
& \left.\left(\delta \bar{\varepsilon}_{4 c} \bar{c}_{44}^{D}\right) \bar{\varepsilon}_{4 c}+\left(\delta \bar{\varepsilon}_{5 c} \bar{c}_{55}^{D}\right) \bar{\varepsilon}_{5 c}+\left(\delta \bar{\varepsilon}_{6 c} \bar{c}_{66}^{D}\right) \bar{\varepsilon}_{6 c}\right] d V
\end{aligned}
$$

Usando a expressão para deformações normal e de cisalhamento para a camada central (2.12), 


$$
\begin{aligned}
\delta H_{m c}=\int_{V}\left[\left(\delta \varepsilon_{1 m c}+z \delta \varepsilon_{1 f c}\right)\right. & \bar{c}_{11}^{D}\left(\varepsilon_{1 m i}+z \varepsilon_{1 f c}\right) \\
+ & \left(\delta \varepsilon_{2 m c}+z \delta \varepsilon_{1 f c}\right) \bar{c}_{12}^{D}\left(\varepsilon_{2 m c}+z \varepsilon_{2 f c}\right) \\
+ & \left(\delta \varepsilon_{1 m i}+z \delta \varepsilon_{1 f c}\right) \bar{c}_{12}^{D}\left(\varepsilon_{2 m c}+z \varepsilon_{2 f c}\right) \\
+ & \left(\delta \varepsilon_{2 m c}+z \delta \varepsilon_{2 f c}\right) \bar{c}_{22}^{D}\left(\varepsilon_{2 m c}+z \varepsilon_{2 f c}\right) \\
& +\left(\delta \varepsilon_{4 c} \bar{c}_{44}^{D} \varepsilon_{4 c}\right)+\left(\varepsilon_{5 c} \bar{c}_{55}^{D} \varepsilon_{5 c}\right) \\
& \left.+\left(\delta \varepsilon_{6 c}+z \delta \kappa_{6 c}\right) \bar{c}_{66}^{D}\left(\varepsilon_{6 c}+z \kappa_{6 c}\right)\right] d V
\end{aligned}
$$

Realizando o mesmo processo que o caso anterior para as camada superior e inferior, podemos encontrar a equação para a camada central definida por,

$$
\begin{array}{r}
\delta H_{m c}=\int_{x} \int_{y}\left[I _ { 3 c } \left(\delta \varepsilon_{1 m c} \bar{c}_{11}^{D} \varepsilon_{1 m c}+\delta \varepsilon_{2 m c} \bar{c}_{12}^{D} \varepsilon_{1 m c}+\delta \varepsilon_{1 m c} \bar{c}_{12}^{D} \varepsilon_{2 m c}+\delta \varepsilon_{2 m c} \bar{c}_{22}^{D} \varepsilon_{2 m c}+\right.\right. \\
\left.\delta \varepsilon_{4 c} \bar{c}_{44}^{D} \varepsilon_{4 c}+\delta \varepsilon_{5 c} \bar{c}_{55}^{D} \delta \varepsilon_{5 c}+\delta \varepsilon_{6 c} \bar{c}_{66}^{D} \varepsilon_{6 c}\right)+I_{4 c}\left(\delta \varepsilon_{1 f c} \bar{c}_{11}^{D} \varepsilon_{1 f c}+\delta \varepsilon_{2 f c} \bar{c}_{12}^{D} \varepsilon_{1 f c}+\right. \\
\left.\left.\delta \varepsilon_{1 f c} \bar{c}_{12}^{D} \varepsilon_{2 f c}+\delta \varepsilon_{2 f c} \bar{c}_{22}^{D} \varepsilon_{2 f c}+\delta \kappa_{6 c} \bar{c}_{66}^{D} \kappa_{6 c}\right)\right] d x d y
\end{array}
$$

onde $I_{3 c}$ e $I_{4 c}$ são escritas iguais a $I_{3 i}$ e $I_{4 i}$ respectivamente.

As contribuições eletromecânicas (ou piezelétricas) para o trabalho virtual das forças internas para as camadas externas podem ser escritas como,

$$
\delta H_{m e i}=\int_{V}\left(\delta \varepsilon^{t} \mathbf{h}^{t} \mathbf{D}+\delta \boldsymbol{D}^{t} \overline{\mathbf{h}} \mathcal{\varepsilon}\right) d V
$$

Usando as expressões para as deformações (2.10), tem-se que

$$
\delta H_{m e i}=\int_{V}\left[\left(\delta \varepsilon_{1 m i} \bar{h}_{31}+\delta \varepsilon_{2 m i} \bar{h}_{32}\right)+\left(z-z_{i}\right)\left(\delta \varepsilon_{1 f i} \bar{h}_{31}+\delta \varepsilon_{2 f i} \bar{h}_{32}\right)\right] D_{3}+
$$




$$
\delta D_{3}\left[\left(\bar{h}_{31} \varepsilon_{1 m i}+\bar{h}_{32} \varepsilon_{2 m i}\right)+\left(z-z_{i}\right)\left(\bar{h}_{31} \varepsilon_{1 m i}+\bar{h}_{32} \varepsilon_{2 f i}\right)\right] d V
$$

Utilizando as integrações propostas anteriormente para a secão transversal, podemos reduzir a equação para,

$$
\begin{array}{r}
\delta H_{m e i}=\int_{x} \int_{y} I_{3 i}\left[\left(\delta \varepsilon_{1 m i} \bar{h}_{31}+\delta \varepsilon_{2 m i} \bar{h}_{32}\right) D_{3}+\delta D_{3}\left(\bar{h}_{31} \varepsilon_{1 m i}+\bar{h}_{32} \varepsilon_{2 f i}\right)\right]+ \\
I_{4 i}\left[\left(\delta \varepsilon_{1 f i} \bar{h}_{31}+\delta \varepsilon_{2 f i} \bar{h}_{32}\right) D_{3}+\delta D_{3}\left(\bar{h}_{31} \varepsilon_{1 f i}+\bar{h}_{32} \varepsilon_{2 f i}\right)\right] d x d y
\end{array}
$$

Escrevendo a equação da contribuição eletromecânica para a camada central e realizando o mesmo procedimento, utilizando as expressões para as deformações (2.12), tem-se que

$$
\begin{aligned}
\delta H_{m e c}= & \int_{x} \int_{y} I_{3 c}\left[\left(\delta \varepsilon_{1 m c} \bar{h}_{31}+\delta \varepsilon_{2 m c} \bar{h}_{32}+\delta \varepsilon_{5}^{c} \bar{h}_{35}\right) D_{3}+\delta D_{3}\left(\bar{h}_{31} \varepsilon_{1 m c}+\bar{h}_{32} \varepsilon_{2 f c}\right)\right]+ \\
& I_{4 c}\left[\left(\delta \varepsilon_{1 f c} \bar{h}_{31}+\delta \varepsilon_{2 f c} \bar{h}_{32}+\delta \mathcal{E}_{5}^{c} \bar{h}_{35}\right) D_{3}+\delta D_{3}\left(\bar{h}_{31} \varepsilon_{1 f c}+\bar{h}_{32} \varepsilon_{2 f c}\right)\right] d x d y
\end{aligned}
$$

A contribuição dielétrica para o trabalho virtual das forças internas pode ser escrita igual para todas as camadas como sendo,

$$
\delta H_{e i}=\int_{V}\left(\delta \mathbf{D}^{t} \overline{\boldsymbol{\beta}}^{\varepsilon} \mathbf{D}\right) d V
$$

desta forma, substituindo os valores das equações 2.1.2 podemos reescrever a equação como sendo,

$$
\delta H_{e i}=\int_{V}\left(\delta D_{3 i}^{t} \overline{\beta_{33}} D_{3 i}\right) d V
$$

Analogamente aos casos anteriores realizamos uma integração na seção transversal, obtendo 


$$
\delta H_{e i}=\int_{x} \int_{y} I_{3 i}\left(\delta D_{3 i}^{t} \overline{\beta_{33}} D_{3 i}\right) d y d x
$$

\subsection{Formulação em Elementos Finitos}

Usando as expressões para trabalho virtual apresentadas previamente, um modelo de elementos finitos para uma placa sanduíche piezoelétrica é desenvolvido. Funções lineares de Lagrange são consideradas para os deslocamentos axiais, $u_{t}, v_{t}, u_{b}$ e $v_{b}$, e deslocamentos elétricos em cada camada, $D_{3 t}, D_{3 c}$ e $D_{3 b}$. Para as deflexões transversais $w$, funções de Hermite cúbicas são consideradas. Desta forma, um elemento finito com 4 nós por elemento e sete graus de liberdade por nó pode ser obtido, conforme mostrado na Figura 2.2 .

\subsubsection{Discretização de deslocamentos e deformações}

Os graus de liberdade mecânicos são agrupados em um vetor $\mathbf{u}_{n}$ tal que

$$
\mathbf{u}_{n}=\left[\begin{array}{llll}
u_{g}^{1} & u_{g}^{2} & u_{g}^{3} & u_{g}^{4}
\end{array}\right]^{t}
$$

Pode-se escrever $u_{g}^{j}$ como sendo,

$$
\mathbf{u}_{g}^{j}=\left[\begin{array}{lllllll}
u_{t}^{j} & v_{t}^{j} & w^{j} & w_{x}^{j} & w_{y}^{j} & u_{b}^{j} & v_{b}^{j}
\end{array}\right]^{t}
$$

onde $j=1,2,3$ e 4 representam os nós do elemento considerado.

Os deslocamentos de cada camada podem ser escritos em termos dos graus de liberdade elementares,

$$
\mathbf{u}_{j}=\mathbf{N}_{u} \mathbf{u}_{n}
$$

sendo $\mathbf{N}_{u}$ definido como, 

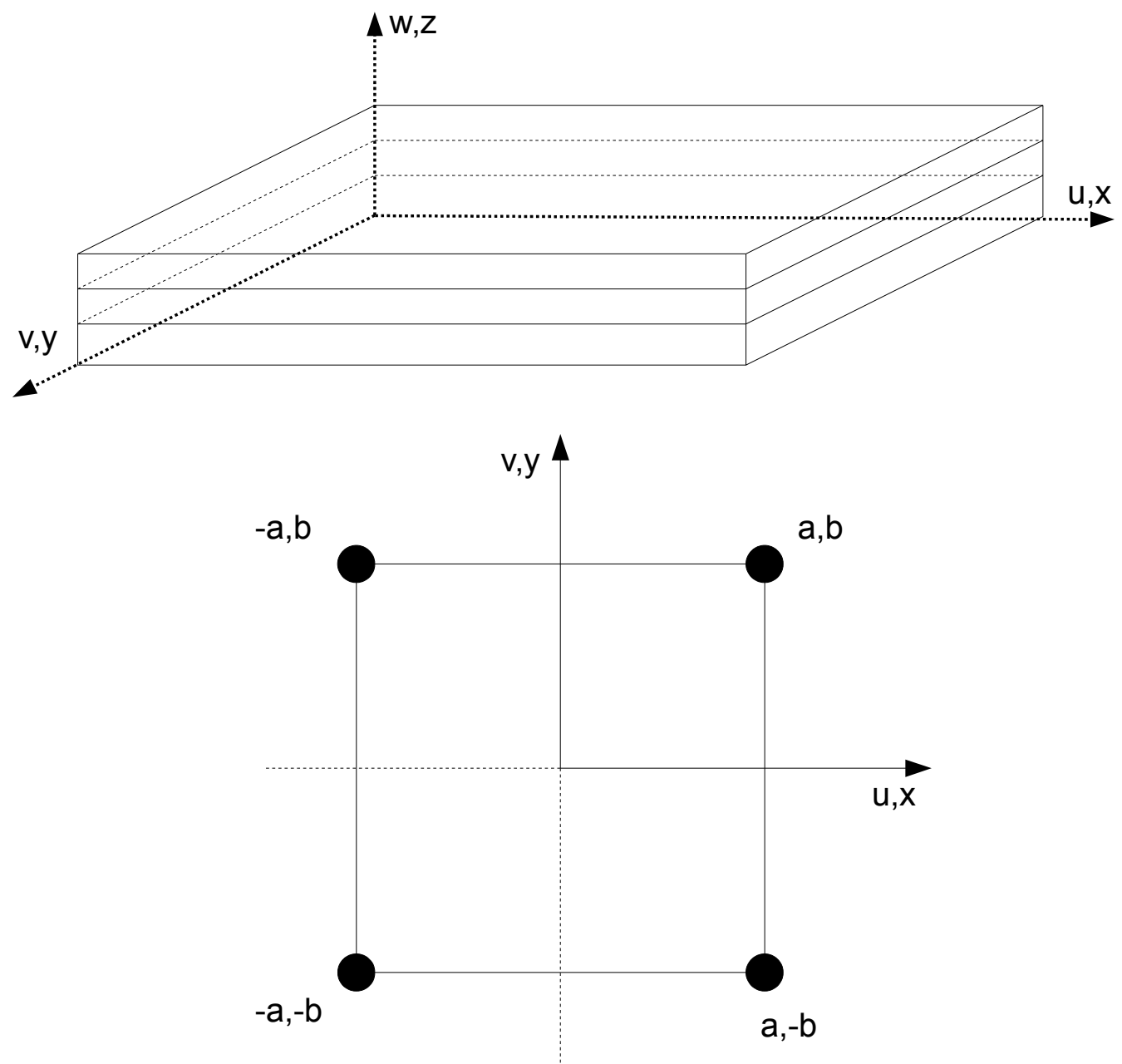

Figura 2.2: Configuração de elemento finito com 4 nós utilizado na elaboração do modelo. 


$$
\mathbf{N}_{u}=\left[\begin{array}{llll}
N_{g}^{1} & N_{g}^{2} & N_{g}^{3} & N_{g}^{4}
\end{array}\right]
$$

e onde

$$
\mathbf{N}_{g}^{j}=\left[\begin{array}{lllllll}
N_{u t}^{j} & N_{v t}^{j} & N_{w} & N_{w x} & N_{w y} & N_{u b}^{j} & N_{v b}^{j}
\end{array}\right]
$$

Discretizando os 4 nós considerados, podemos escrever,

$$
\begin{aligned}
\mathbf{N}_{u t}^{j} & =\left[\begin{array}{llllllll}
N_{j} & 0 & 0 & 0 & 0 & 0 & 0 & 0
\end{array}\right] \\
\mathbf{N}_{v t}^{j} & =\left[\begin{array}{llllllll}
0 & N_{j} & 0 & 0 & 0 & 0 & 0 & 0
\end{array}\right] \\
\mathbf{N}_{u b}^{j} & =\left[\begin{array}{llllllll}
0 & 0 & 0 & 0 & 0 & 0 & N_{j} & 0
\end{array}\right] \\
\mathbf{N}_{v b}^{j} & =\left[\begin{array}{llllllll}
0 & 0 & 0 & 0 & 0 & 0 & 0 & N_{j}
\end{array}\right] \\
\mathbf{N}_{w} & =\left[\begin{array}{llllllll}
0 & 0 & N_{5}^{j} & N_{6}^{j} & N_{7}^{j} & 0 & 0 & 0
\end{array}\right]
\end{aligned}
$$

Para a camada central (central), podemos escrever,

$$
\begin{aligned}
& \mathbf{N}_{u c}^{j}=\frac{N_{u b}^{j}+N_{u t}^{j}}{2}+\frac{h_{d}}{4} \frac{\partial N_{w}}{\partial x} \\
& \mathbf{N}_{v c}^{j}=\frac{N_{v b}^{j}+N_{v t}^{j}}{2}+\frac{h_{d}}{4} \frac{\partial N_{w}}{\partial y} \\
& \mathbf{N}_{\phi_{x}}=\frac{N_{u t}^{j}-N_{u b}^{j}}{h_{c}}+\frac{h_{m}}{h_{c}} \frac{\partial N_{w}}{\partial x} \\
& \mathbf{N}_{\phi_{y}}=\frac{N_{v t}^{j}-N_{v b}^{j}}{h_{c}}+\frac{h_{m}}{h_{c}} \frac{\partial N_{w}}{\partial y}
\end{aligned}
$$

As funções de forma $N_{j}(j=1, \ldots, 6)$ são escritas como 


$$
\begin{aligned}
N_{1} & =\frac{1}{4}(1-\epsilon)(1+\eta) \\
N_{2} & =\frac{1}{4}(1+\epsilon)(1-\eta) \\
N_{3} & =\frac{1}{4}(1+\epsilon)(1+\eta) \\
N_{4} & =\frac{1}{4}(1-\epsilon)(1+\eta) \\
N_{5 j} & =\frac{1}{8}\left(1+\epsilon_{0}\right)\left(1+\eta_{0}\right)\left(2+\epsilon_{0}+\eta_{0}-\epsilon^{2}-\eta^{2}\right) \\
N_{6 j} & =\frac{1}{8} \epsilon_{j}\left(\epsilon_{0}-1\right)\left(1+\eta_{0}\right)\left(1+\epsilon_{0}\right)^{2} \\
N_{7 j} & =\frac{1}{8} \eta_{j}\left(\eta_{0}-1\right)\left(1+\epsilon_{0}\right)\left(1+\eta_{0}\right)^{2}
\end{aligned}
$$

As funções de interpolação de Hermite são da forma cúbica. Para um elemento retangular, estas podem ser escritas em termos das coordenadas naturais tais que, $\epsilon$ e $\eta$ são as já definidas e $\epsilon_{0}=\epsilon \epsilon_{j}$ e $\eta_{0}=\eta \eta_{j}$, sendo que $\epsilon_{j}=x_{j} a$ e $\eta_{j}=y_{j} b$. Observa-se que as variáveis $\epsilon$ e $\eta$ são as coordenadas naturais definidas como: $\epsilon=(x / a)$ e $\eta=(y / b)$ na figura 2.2.

O deslocamento transversal $w$ pode ser escrito como

$$
w(x, y, t)=\sum_{j=1}^{4} w^{j} N_{5 j}(x, y)+w^{j} N_{6 j}(x, y)+w^{j} N_{7 j}(x, y)
$$

De acordo com as expressões (2.11) e (2.13), as deformações generalizadas podem ser escritas em termos dos graus de liberdade elementares. Para as camadas superior e inferior, podemos escrever, 


$$
\begin{aligned}
\varepsilon_{1 m i} & =\mathbf{B}_{1 m i} u_{n} \\
\varepsilon_{1 f i} & =\mathbf{B}_{1 f i} u_{n} \\
\mathcal{E}_{2 m i} & =\mathbf{B}_{2 m i} u_{n} \\
\mathcal{E}_{2 f i} & =\mathbf{B}_{2 f i} u_{n} \\
\varepsilon_{6 i} & =\mathbf{B}_{6 i} u_{n} \\
\kappa_{6 i} & =\boldsymbol{\psi}_{6 i} u_{n}
\end{aligned}
$$

Para a camada central, escrevemos,

$$
\begin{aligned}
\varepsilon_{1 m c} & =\mathbf{B}_{1 m c} u_{n} \\
\varepsilon_{1 f c} & =\mathbf{B}_{1 f c} u_{n} \\
\mathcal{E}_{2 m c} & =\mathbf{B}_{2 m c} u_{n} \\
\mathcal{E}_{2 f c} & =\mathbf{B}_{2 f c} u_{n} \\
\mathcal{E}_{4 c} & =\mathbf{B}_{4} u_{n} \\
\varepsilon_{5 c} & =\mathbf{B}_{5} u_{n} \\
\varepsilon_{6 c} & =\mathbf{B}_{6 c e} u_{n} \\
\kappa_{6 c} & =\mathbf{B}_{6 c k} u_{n}
\end{aligned}
$$

Os operadores de deformação para membrana, flexão e cisalhamento são definidos como, 


$$
\begin{aligned}
\mathbf{B}_{1 m i} & =\frac{\partial N_{u i}}{\partial x} \\
\mathbf{B}_{1 f i} & =\frac{-\partial^{2} N_{w}}{\partial x^{2}} \\
\mathbf{B}_{2 m i} & =\frac{\partial N_{u i}}{\partial y} \\
\mathbf{B}_{2 f i} & =\frac{-\partial^{2} N_{w}}{\partial y^{2}} \\
\mathbf{B}_{6 i} & =\left(\frac{\partial N_{u i}}{\partial y}+\frac{\partial N_{v i}}{\partial x}\right) \\
\boldsymbol{\psi}_{6 i} & =\left(-2 \frac{\partial^{2} N_{w}}{\partial x \partial y}\right)
\end{aligned}
$$

Para os operadores da camada central, temos,

$$
\begin{aligned}
\mathbf{B}_{1 m c} & =\frac{\partial N_{u c}}{\partial x} \\
\mathbf{B}_{1 f c} & =\frac{\partial N_{\phi_{x}}}{\partial x} \\
\mathbf{B}_{2 m c} & =\frac{\partial N_{v c}}{\partial y} \\
\mathbf{B}_{2 f c} & =\frac{\partial N_{\phi_{y}}}{\partial y} \\
\mathbf{B}_{4} & =N_{\phi_{y}}+\frac{\partial N_{w}}{\partial y} \\
\mathbf{B}_{5} & =N_{\phi_{x}}+\frac{\partial N_{w}}{\partial x} \\
\mathbf{B}_{6 c} & =\left(\frac{\partial N_{u c}}{\partial y}+\frac{\partial N_{v c}}{\partial x}\right) \\
\boldsymbol{\psi}_{6 c} & =\left(\frac{\partial N_{\phi_{x}}}{\partial y}+\frac{\partial N_{\phi_{y}}}{\partial x}\right)
\end{aligned}
$$

\subsubsection{Discretização dos deslocamentos elétricos}

Os graus de liberdade elétricos são agrupadas em um vetor coluna $\mathbf{D}_{n}$, definido como

$$
\mathbf{D}_{n}=\left[\begin{array}{llll}
D^{1} & D^{2} & D^{3} & D^{4}
\end{array}\right]^{t}
$$

onde $D$ é definido como, 


$$
D^{j}=\left[\begin{array}{lll}
D_{3 t}^{j} & D_{3 c}^{j} & D_{3 b}^{j}
\end{array}\right] ; \quad j=1: 4
$$

Então o deslocamento elétrico nas camadas piezoelétricas pode ser escrito em termos dos graus de liberdade elétricas elementares,

$$
\mathbf{D}_{3 i}=\mathbf{N}_{D i} \mathbf{D}_{n}
$$

sendo,

$$
\begin{aligned}
& \mathbf{N}_{D t}=\left[\begin{array}{llllllllllll}
N_{1} & 0 & 0 & N_{2} & 0 & 0 & N_{3} & 0 & 0 & N_{4} & 0 & 0
\end{array}\right] \\
& \mathbf{N}_{D c}=\left[\begin{array}{llllllllllll}
0 & N_{1} & 0 & 0 & N_{2} & 0 & 0 & N_{3} & 0 & 0 & N_{4} & 0
\end{array}\right] \\
& \mathbf{N}_{D b}=\left[\begin{array}{llllllllllll}
0 & 0 & N_{1} & 0 & 0 & N_{2} & 0 & 0 & N_{3} & 0 & 0 & N_{4}
\end{array}\right]
\end{aligned}
$$

\subsubsection{Discretização das expressões de trabalho virtual}

Através das equações, (2.43) e (2.44), (2.47) e (2.49), podemos reescrever a expressão do trabalho virtual das forças de inércia como sendo

$$
\delta W_{m i}^{c}=-\delta \mathbf{u}_{n}^{t} \mathbf{M}_{i} \ddot{\mathbf{u}}_{n}
$$

onde $\mathbf{M}_{i}$ é a matriz de massa da $i$-ésima camada. Esta é definida como,

$$
\mathbf{M}_{i}=\int_{x} \int_{y} I_{0 i}\left[\mathbf{N}_{u i}^{t} \mathbf{N}_{u i}+\mathbf{N}_{v i}^{t} \mathbf{N}_{v i}+\mathbf{N}_{w}^{t} \mathbf{N}_{w}\right]+I_{2 i}\left[\mathbf{N}_{w, x}^{t} \mathbf{N}_{w, x}+\mathbf{N}_{w, y}^{t} \mathbf{N}_{w, y}\right] d y d x
$$

onde $\mathbf{N}_{w, x}$ e $\mathbf{N}_{w, y}$ são as derivadas de $\mathbf{N}_{w}$ em função da variável $x$ e $y$, respectivamente. Para a camada central, devemos escrever, 


$$
\begin{gathered}
\delta W_{m c}^{c}=-\delta \mathbf{u}_{n}^{t} \mathbf{M}_{i} \ddot{\mathbf{u}}_{n} \\
\mathbf{M}_{c}=\int_{x} \int_{y} I_{0 c}\left[\mathbf{N}_{u c}^{t} \mathbf{N}_{u c}+\mathbf{N}_{v c}^{t} \mathbf{N}_{v c}+\mathbf{N}_{w}^{t} \mathbf{N}_{w}\right]+I_{2 c}\left[\mathbf{N}_{\phi_{x}}^{t} \mathbf{N}_{\phi_{x}}+\mathbf{N}_{\phi_{y}}^{t} \mathbf{N}_{\phi_{y}}\right] d y d x
\end{gathered}
$$

onde os vetores de interpolação foram definidos na seção anterior. A contribuição da energia potencial mecânica para o trabalho virtual das forças eletromecânicas pode ser discretizada através de (2.51) e (2.53), para as camadas superior e inferior, e (2.52) e (2.54), para a camada central. Assim,

$$
\delta H_{m i}=\delta \mathbf{u}_{n}^{t} \mathbf{K}_{m i} \mathbf{u}_{n}
$$

sendo $\mathbf{K}_{m i}$ a matriz de rigidez mecânica da $i$-ésima camada escrita, para as camadas superior e inferior, como

$$
\begin{aligned}
\mathbf{K}_{m i}=\int_{x} \int_{y} I_{3 i}\left[\mathbf{B}_{1 m i}^{t} \bar{c}_{11} \mathbf{B}_{1 m i}+\mathbf{B}_{2 m i}^{t} \bar{c}_{12} \mathbf{B}_{1 m i}+\mathbf{B}_{1 m i}^{t} \bar{c}_{22} \mathbf{B}_{2 m i}+\right. \\
\left.\mathbf{B}_{6 m i}^{t} \bar{c}_{66} \mathbf{B}_{6 m i}\right]+I_{4 i}\left[\mathbf{B}_{1 f i}^{t} \bar{c}_{11} \mathbf{B}_{1 f i}+\mathbf{B}_{2 f i}^{t} \bar{c}_{12} \mathbf{B}_{1 f i}+\right. \\
\left.\mathbf{B}_{1 f i}^{t} \bar{c}_{12} \mathbf{B}_{2 f i}+\mathbf{B}_{2 f i}^{t} \bar{c}_{22} \mathbf{B}_{2 f i}+\boldsymbol{\psi}_{6 i}^{t} \bar{c}_{66} \boldsymbol{\psi}_{6 i}\right] d y d x
\end{aligned}
$$

e, para a camada central,

$$
\delta H_{m c}=\delta \mathbf{u}_{n}^{t} \mathbf{K}_{m c} \mathbf{u}_{n}
$$




$$
\begin{gathered}
\mathbf{K}_{m c}=\int_{x} \int_{y} I_{3 c}\left[\mathbf{B}_{1 m c}^{t} \bar{c}_{11} \mathbf{B}_{1 m c}+\mathbf{B}_{2 m c}^{t} \bar{c}_{12} \mathbf{B}_{1 m c}+\mathbf{B}_{1 m c}^{t} \bar{c}_{12} \mathbf{B}_{2 m c}+\mathbf{B}_{2 m c} \bar{c}_{22} \mathbf{B}_{2 m c}\right. \\
\left.\mathbf{B}_{4}^{t} \bar{c}_{44} \mathbf{B}_{4}+\mathbf{B}_{5}^{t} \bar{c}_{55} \mathbf{B}_{5}+\mathbf{B}_{6 c}^{t} \bar{c}_{66} \mathbf{B}_{6 c}\right]+ \\
I_{4 c}\left[\mathbf{B}_{1 f c}^{t} \bar{c}_{11} \mathbf{B}_{1 f c}+\mathbf{B}_{2 f c}^{t} \bar{c}_{12} \mathbf{B}_{1 f c}+\mathbf{B}_{1 f c}^{t} \bar{c}_{12} \mathbf{B}_{1 f c}+\right. \\
\left.\mathbf{B}_{1 f c}^{t} \bar{c}_{12} \mathbf{B}_{2 f c}+\mathbf{B}_{2 f c}^{t} \bar{c}_{12} \mathbf{B}_{2 f c}+\mathbf{B}_{5}^{t} \bar{c}_{55} \mathbf{B}_{5}+\boldsymbol{\psi}_{6 c}^{t} \bar{c}_{66} \boldsymbol{\psi}_{6 c}\right] d y d x
\end{gathered}
$$

Através da equação (2.53), para as camadas externas, e da equação (2.54), para a camada central, e as expressões discretizadas para as deformações (2.53) e deslocamentos elétricos (2.57), as contribuições piezoelétricas para o trabalho virtual das forças eletromecânicas internas são reescritas por,

$$
\delta H_{m e i}=-\delta \mathbf{u}_{n}^{t} \mathbf{K}_{m e i} \mathbf{D}_{n}-\delta \mathbf{D}_{n}^{t} \mathbf{K}_{m e i}^{t} \mathbf{u}_{n}
$$

onde $\mathbf{K}_{m e i}$ é a matriz de rigidez eletromecânica (piezoelétrica) da $i$-ésima camada definida como sendo, para as camadas externas

$$
\begin{aligned}
& \mathbf{K}_{m e i}=\int_{x} \int_{y} I_{3 i}\left[\left(\mathbf{B}_{1 m i}^{t} \bar{h}_{31}+\mathbf{B}_{2 m i}^{t} \bar{h}_{32}\right) \mathbf{N}_{D i}+\mathbf{N}_{D i}^{t}\left(\bar{h}_{31} \mathbf{B}_{1 m i}+\bar{h}_{32} \mathbf{B}_{2 m i}\right)\right]+ \\
& \qquad I_{4 i}\left[\left(\mathbf{B}_{1 f i}^{t} \bar{h}_{31}+\mathbf{B}_{2 f i}^{t} \bar{h}_{32}\right) \mathbf{N}_{D i}+\mathbf{N}_{D i}^{t}\left(\bar{h}_{31} \mathbf{B}_{1 f i}+\bar{h}_{32} \mathbf{B}_{2 f i}\right)\right] d y d x \\
& \text { e, para a camada central, }
\end{aligned}
$$

$$
\begin{array}{r}
\mathbf{K}_{m e c}=\int_{x} \int_{y} I_{3 c}\left[\left(\mathbf{B}_{1 m c}^{t} \bar{h}_{31}+\mathbf{B}_{2 m c}^{t} \bar{h}_{32}+\mathbf{B}_{5}^{t} \bar{h}_{35}\right) \mathbf{N}_{D c}+\mathbf{N}_{D c}^{t}\left(\bar{h}_{31} \mathbf{B}_{1 m c}+\bar{h}_{32} \mathbf{B}_{2 m c}+\bar{h}_{35} \mathbf{B}_{5}\right)\right]+ \\
I_{4 c}\left[\left(\mathbf{B}_{1 f c}^{t} \bar{h}_{31}+\mathbf{B}_{2 f c}^{t} \bar{h}_{32}\right) \mathbf{N}_{D c}+\mathbf{N}_{D c}^{t}\left(\bar{h}_{31} \mathbf{B}_{1 f c}+\bar{h}_{32} \mathbf{B}_{2 f c}\right)\right] d y d x
\end{array}
$$


As contribuições dielétricas para o trabalho virtual das forças internas podem ser descritas através das equações (2.41) e (2.57), dando origem a

$$
\delta H_{e i}=\delta \mathbf{D}_{n}^{t} \mathbf{K}_{e i} \mathbf{D}_{n}
$$

onde $\mathbf{K}_{e i}$ é a matriz de rigidez dielétrica, que pode ser descrita igual para todas as camadas como sendo,

$$
\mathbf{K}_{e i}=\int_{x} \int_{y} I_{3 i} \mathbf{N}_{D i}^{t} \beta_{33} \mathbf{N}_{D i} d y d x \quad \text { e } \quad \mathbf{K}_{e c}=\int_{x} \int_{y} I_{3 c} \mathbf{N}_{D c}^{t} \beta_{33} \mathbf{N}_{D c} d y d x
$$

\subsection{Equações de movimento}

Com os trabalhos virtuais discretizados, pode-se agora substitui-los no princípio variacional (2.18) e obter as equações de movimento da viga sanduíche, a nível elementar. Assim,

$$
\delta \mathbf{u}_{n}^{t}\left(\mathbf{M}^{e} \ddot{\mathbf{u}}_{n}+\mathbf{K}_{m}^{e} \mathbf{u}_{n}-\mathbf{K}_{m e}^{e} \mathbf{D}_{n}-\mathbf{F}^{e}\right)+\delta \mathbf{D}_{n}^{t}\left(-\mathbf{K}_{m e}^{e t} \mathbf{u}_{n}+\mathbf{K}_{e}^{e} \mathbf{D}_{n}\right)=0
$$

onde as matrizes elementares de massa e rigidez e o vetor das forças mecânicas são dados por,

$$
\mathbf{M}^{e}=\sum_{i=1}^{3} \mathbf{M}_{i} ; \mathbf{K}_{m}^{e}=\sum_{i=1}^{3} \mathbf{K}_{m i} ; \mathbf{K}_{m e}^{e}=\sum_{i=1}^{3} \mathbf{K}_{m e i} ; \mathbf{K}_{e}^{e}=\sum_{i=1}^{3} \mathbf{K}_{e i} ; \mathbf{F}^{e}=\sum_{i=1}^{3} \mathbf{F}_{i}
$$

ou seja, uma soma das contribuições de cada camada. Assim, as equações de movimento podem ser escritas como, 


$$
\left[\begin{array}{cc}
\mathbf{M}^{e} & 0 \\
0 & 0
\end{array}\right]\left\{\begin{array}{c}
\ddot{\mathbf{u}}_{n} \\
\ddot{\mathbf{D}}_{n}
\end{array}\right\}+\left[\begin{array}{cc}
0 & 0 \\
0 & 0
\end{array}\right]\left\{\begin{array}{c}
\dot{\mathbf{u}}_{n} \\
\dot{\mathbf{D}}_{n}
\end{array}\right\}+\left[\begin{array}{cc}
\mathbf{K}_{m}^{e} & -\mathbf{K}_{m e}^{e} \\
-\mathbf{K}_{m e}^{e t} & \mathbf{K}_{e}^{e}
\end{array}\right]\left\{\begin{array}{c}
\mathbf{u}_{n} \\
\mathbf{D}_{n}
\end{array}\right\}=\left\{\begin{array}{c}
\mathbf{F}^{e} \\
0
\end{array}\right\}
$$

Como temos vários elementos finitos em uma estrutura, podemos reunir todos os elementos e escrever a equação de movimento global como sendo,

$$
\left[\begin{array}{cc}
\mathbf{M} & 0 \\
0 & 0
\end{array}\right]\left\{\begin{array}{c}
\ddot{\mathbf{u}} \\
\ddot{\mathbf{D}}
\end{array}\right\}+\left[\begin{array}{ll}
0 & 0 \\
0 & 0
\end{array}\right]\left\{\begin{array}{c}
\dot{\mathbf{u}} \\
\dot{\mathbf{D}}
\end{array}\right\}+\left[\begin{array}{cc}
\mathbf{K}_{m} & -\mathbf{K}_{m e} \\
-\mathbf{K}_{m e}^{t} & \mathbf{K}_{e}
\end{array}\right]\left\{\begin{array}{l}
\mathbf{u} \\
\mathbf{D}
\end{array}\right\}=\left\{\begin{array}{l}
\mathbf{F} \\
0
\end{array}\right\}
$$

onde u e D são os graus de liberdade mecânicos e elétricos globais. Levando em consideração os eletrodos que recobrem as superfícies superior e inferior das camadas, teremos que os deslocamentos elétricos e nós selecionados na camada, na região do eletrodo, devem ser iguais. Isto pode ser feito pela seguinte equação,

$$
\mathbf{D}=\mathbf{L}_{p} \mathbf{D}_{p}
$$

onde $\mathbf{L}_{p}$ representa uma matriz binária e $\mathbf{D}_{p}$ é o vetor dos deslocamentos elétricos em cada uma das camada piezoelétricas, conectadas por um mesmo eletrodo

$$
\mathbf{D}_{p}=\left[\begin{array}{llll}
D_{p 1} & D_{p 2} & \cdots & D_{p n}
\end{array}\right]^{t}
$$

Substituindo a equação (2.78) na equação (2.77), pode-se reescrever as equações de movimento como

$$
\left[\begin{array}{cc}
\mathbf{M} & 0 \\
0 & 0
\end{array}\right]\left\{\begin{array}{c}
\ddot{\mathbf{u}} \\
\ddot{\mathbf{D}}_{p}
\end{array}\right\}+\left[\begin{array}{cc}
0 & 0 \\
0 & 0
\end{array}\right]\left\{\begin{array}{c}
\dot{\mathbf{u}} \\
\dot{\mathbf{D}}_{p}
\end{array}\right\}+\left[\begin{array}{cc}
\mathbf{K}_{m} & -\overline{\mathbf{K}}_{m e} \\
-\overline{\mathbf{K}}_{m e}^{t} & \overline{\mathbf{K}}_{e}
\end{array}\right]\left\{\begin{array}{c}
\mathbf{u} \\
\mathbf{D}_{p}
\end{array}\right\}=\left\{\begin{array}{l}
\mathbf{F} \\
0
\end{array}\right\}
$$


sendo que as matrizes de rigidez piezoelétricas e dielétricas são modificadas para levar em conta o efeito do eletrodo, tal que

$$
\overline{\mathbf{K}}_{m e}=\mathbf{K}_{m e} \mathbf{L}_{p} ; \overline{\mathbf{K}}_{e}=\mathbf{L}_{p}^{t} \mathbf{K}_{e} \mathbf{L}_{p}
$$

Através das equações de movimento podemos perceber que, quando fazemos $\mathbf{D}_{p}=0$, caracterizando o caso de circuito aberto (OC), observa-se a redução da equação para o caso puramente mecânico,

$$
\mathbf{M u ̈}+\mathbf{K}_{m} \mathbf{u}=\mathbf{F}
$$

No caso de pastilhas em circuito fechado (SC), para as quais $\mathbf{D}_{p} \neq 0$, a segunda linha da equação (2.81) pode ser condensada estaticamente, resolvendo-a para $\mathbf{D}_{p} \mathrm{em}$ função de u. Isto equivale a afirmar que o deslocamento elétrico nas pastilhas em SC é totalmente determinado pelo estado de deslocamento da estrutura. Substituindo-se a solução de $\mathbf{D}_{p}$ na primeira linha da equação (2.80), obtém-se novamente uma equação diferencial "puramente mecânica" em u, na qual se observa uma redução equivalente na rigidez da estrutura, em relação ao caso OC, devido a ausência de acúmulo de carga elétrica nos eletrodos das pastilhas neste caso, tal que

$$
\mathbf{M} \ddot{\mathbf{u}}+\left(\mathbf{K}_{m}-\overline{\mathbf{K}}_{m e} \overline{\mathbf{K}}_{e}^{-1} \overline{\mathbf{K}}_{m e}^{t}\right) \mathbf{u}=\mathbf{F}
$$

\subsection{Validação do modelo proposto}

Apresentamos aqui alguns resultados numéricos com cerâmicas piezoelétricas em extensão e em cisalhamento conectados em circuito aberto, denominado $\mathrm{OC}$ e em circuito fechado, denominado SC, para validação do modelo de placa proposto neste trabalho. 


\subsection{Pastilhas em Extensão}

Para o estudo das pastilhas em extensão consideraremos a configuração apresentada em dissertação de mestrado [42]. A estrutura estudada foi uma viga com $220 \mathrm{~mm}$ de comprimento com 25mm de largura sendo o elemento piezoelétrico acoplado em sua superfície, localizado a $10 \mathrm{~mm}$ do seu engaste. Neste caso, a viga considerada, tem somente uma de suas extremidades engastada.

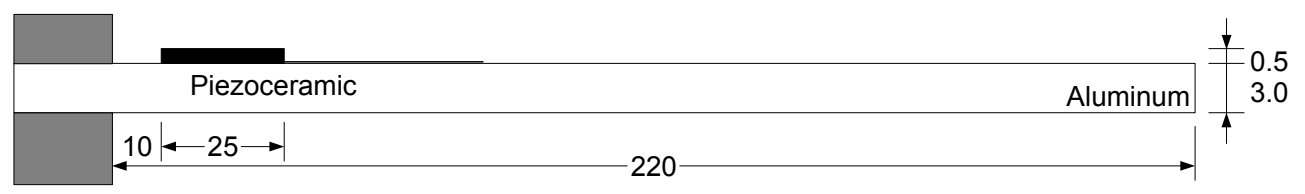

Figura 2.3: Configuração para análise de piezo em extensão

Nesta configuração consideramos cerâmicas piezoelétricas feitas de PZT-5H e as propriedades consideradas do material piezoelétrico foram: $\bar{c}_{11}^{D}=\bar{c}_{22}^{D}=97.767 \mathrm{GPa}$; $\bar{c}_{12}^{D}=50.774 \mathrm{GPa} ; \bar{c}_{44}^{D}=\bar{c}_{55}^{D}=42.217 \mathrm{GPa} ; \rho=7500 \mathrm{~kg} \mathrm{~m}^{-3}$; constante de acoplamento piezoelétrica $\bar{h}_{31}=-1.352010^{9} \mathrm{~N} \mathrm{C}^{-1}$ e e constante dielétrica $\bar{\beta}_{33}^{\mathcal{E}}=57.83010^{6} \mathrm{~m} \mathrm{~F}^{-1}$. Para a viga de aluminio, as propriedades do material são definidas por: Módulo de Young $70.3 \mathrm{GPa}$ e densidade $2710 \mathrm{~kg} \mathrm{~m}^{-3}$, um amortecimento viscoso de $0.5 \%$ foi considerado.

A tabela 2.2 apresenta uma comparação das frequências em circuito aberto (OC) e a tabela 2.3 em circuito fechado (SC) para a estrutura descrita utilizando o modelo de viga e o modelo proposto neste trabalho, modelo de placa.

\subsection{Pastilhas em Cisalhamento}

Para o estudo das pastilhas em cisalhamento consideraremos duas configurações. A primeira configuração foi proposta por Deü e Benjeddou [19], neste trabalho foi considerado uma placa sanduíche quadrada, com camadas Graphite- Epoxy (GE) e PZT-5H, dispostas 
Tabela 2.2: Tabela com os resultados obtidos para comparação de viga em circuito aberto.

\begin{tabular}{|c|c|c|c|}
\hline & Modelo Placa & Modelo Viga & Diferença \\
\hline & Frequencia em OC $(\mathrm{Hz})$ & Frequencia em OC $(\mathrm{Hz})$ & $\%$ \\
\hline Primeiro Modo & 54.9 & 54.1 & +1.47 \\
\hline Segundo Modo & 355 & 334 & +6.28 \\
\hline Terceiro Modo & 1010 & 992 & +1.81 \\
\hline Quarto Modo & 1960 & 1770 & +10.73 \\
\hline Quinto Modo & 3190 & 2890 & +10.38 \\
\hline
\end{tabular}

Tabela 2.3: Tabela com os resultados obtidos para comparação de viga em circuito fechado.

\begin{tabular}{|c|c|c|c|}
\hline & Modelo Placa & Modelo Viga & Diferença \\
\hline & Frequencia em SC (Hz) & Frequencia em SC (Hz) & $\%$ \\
\hline Primeiro Modo & 54.5 & 53.1 & +2.63 \\
\hline Segundo Modo & 354 & 332 & +6.62 \\
\hline Terceiro Modo & 1000 & 920 & +8.69 \\
\hline Quarto Modo & 1960 & 1770 & +10.73 \\
\hline Quinto Modo & 3190 & 2880 & +10.76 \\
\hline
\end{tabular}

com GE 0/PZT-5H/GE 0, sendo a camada de PZT-5H polarizada na direção x. No caso proposto foram consideradas as dimensões para a espessura total de $\mathrm{H}=1 \mathrm{~cm}$, com 0,4 $\mathrm{cm}$ para cada camada GE e $0,2 \mathrm{~cm}$ para a camada de $\mathrm{PZT}$, e comprimento $\mathrm{L}=10 \mathrm{H}$, totalizando $10 \mathrm{~cm}$ de lado. As propriedades do PZT-5H utilizadas foram $c_{11}^{E}=c_{22}^{E}=126 \mathrm{GPa}$; $c_{33}^{E}=117 \mathrm{GPa} ; c_{12}^{E}=79.5 \mathrm{GPa} ; c_{13}^{E}=c_{23}^{E}=84.1 \mathrm{GPa} ; c_{44}^{E}=c_{55}^{E}=c_{66}^{E}=23 \mathrm{GPa}$; $e_{15}=e_{24}=17 \mathrm{Cm}^{-2} ; e_{31}=e_{32}=-6.5 \mathrm{Cm}^{-2} ; e_{33}=23.3 \mathrm{Cm}^{-2} ; \varepsilon_{11}=\varepsilon_{22}=15.03 \mathrm{nFm} \mathrm{F}^{-1}$; $\varepsilon_{33}=13 n \mathrm{Fm}^{-1} ; \rho_{p z t}=7500 \mathrm{~kg} \cdot \mathrm{m}^{-3}$. Para o Graphite - Epoxy, as propriedades utilizadas foram: $c_{11}=183.443 \mathrm{GPa} ; c_{22}=c_{33}=11.662 \mathrm{GPa} ; c_{12}=c_{13}=4.363 \mathrm{GPa}$; $c_{23}=3.918 \mathrm{GPa} ; c_{44}=2.870 \mathrm{GPa} ; c_{55}=c_{66}=2.870 \mathrm{GPa} ; \rho_{G E}=1580 \mathrm{kgm}^{-3}$. As condições de contorno utilizadas neste estudo foram para a placa simplesmente apoiada (SS - Simply Supported). Para esta análise foram utilizadas as frequencias naturais admensionais, para isto utilizou-se a equação $\lambda_{x, y}=\omega_{x, y} L^{2} \sqrt{\rho_{p z t} / c_{55}^{E p z t}}$.

A tabela 2.4 apresenta uma comparação das frequências em circuito aberto (OC) entre o modelo proposto por Deü e Benjeddou [19] e o modelo desenvolvido neste trabalho, para a estrutura acima descrita. 


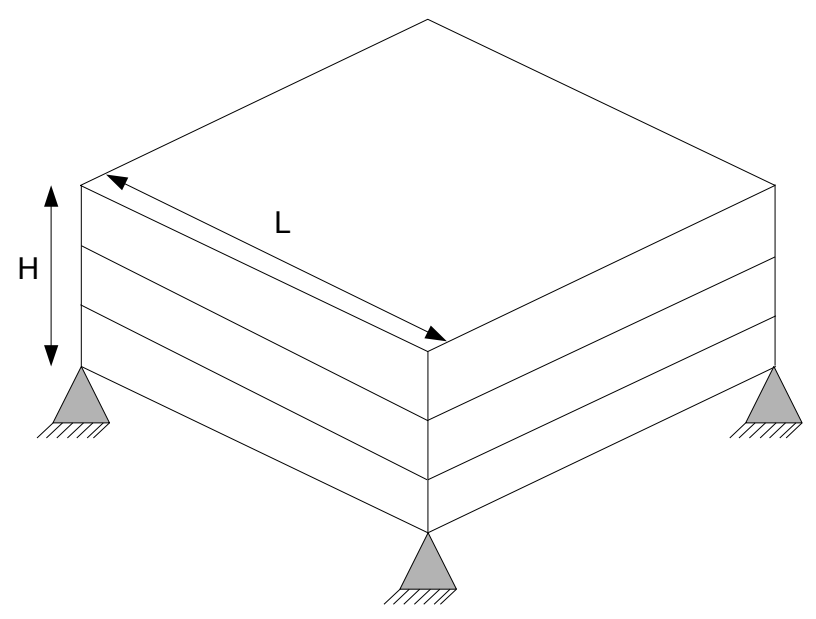

Figura 2.4: Placa sanduíche simplesmente apoiada com camada piezelétrica em cisalhamento

Tabela 2.4: Tabela com os resultados obtidos para comparação de placa em circuito aberto.

\begin{tabular}{|c|c|c|c|}
\hline & Modelo Placa & Referência [19] & Diferença \\
\hline & Frequencia em OC $(\mathrm{Hz})$ & Frequencia em OC $(\mathrm{Hz})$ & $\%$ \\
\hline$\lambda_{1,1}$ & 14.47 & 13.56 & +6.71 \\
\hline$\lambda_{1,2}$ & 21.15 & 19.84 & +6.60 \\
\hline$\lambda_{1,3}$ & 34.92 & 31.80 & +9.81 \\
\hline$\lambda_{2,1}$ & 45.11 & 41.26 & +9.33 \\
\hline$\lambda_{2,2}$ & 50.60 & 45.02 & +12.39 \\
\hline$\lambda_{1,4}$ & 51.61 & 47.88 & +7.79 \\
\hline$\lambda_{2,3}$ & 52.64 & 52.89 & -0.47 \\
\hline
\end{tabular}


Tabela 2.5: Tabela com os resultados obtidos para comparação de placa em circuito fechado.

\begin{tabular}{|c|c|c|c|}
\hline & Modelo Placa & Referência [19] & Diferença \\
\hline & Frequencia em SC (Hz) & Frequencia em SC $(\mathrm{Hz})$ & $\%$ \\
\hline$\lambda_{1,1}$ & 14.47 & 13.49 & +7.26 \\
\hline$\lambda_{1,2}$ & 21.15 & 19.77 & +6.98 \\
\hline$\lambda_{1,3}$ & 34.92 & 31.73 & +10.05 \\
\hline$\lambda_{2,1}$ & 45.11 & 40.68 & +10.88 \\
\hline$\lambda_{2,2}$ & 50.60 & 44.44 & +13.86 \\
\hline$\lambda_{1,4}$ & 51.61 & 47.81 & +7.94 \\
\hline$\lambda_{2,3}$ & 52.64 & 52.33 & +0.59 \\
\hline
\end{tabular}

Na tabela 2.5 apresentamos os resultados em circuito fechado (SC) para a estrutura descrita utilizando o modelo proposto por Deü e Benjeddou [19] e o modelo proposto,

Uma segunda análise foi realizada utilizando a configuração proposta por Baillargeon e Vel [7], neste trabalho foi considerado uma placa, onde o comprimento na direção finita é de 250mm e 2,5mm na outra direção, com GE e PZT-5A, dispostos na forma de GE 0/PZT-5A/GE 0, sendo a camada de PZT-5A polarizada na direção x. As propriedades do PZT-5A utilizadas foram $c_{11}^{E}=c_{22}^{E}=99.201 \mathrm{GPa} ; c_{33}^{E}=86.856 \mathrm{GPa}$; $c_{12}^{E}=54.016 \mathrm{GPa} ; c_{13}^{E}=c_{23}^{E}=50.778 \mathrm{GPa} ; c_{44}^{E}=c_{55}^{E}=c_{66}^{E}=22.600 \mathrm{GPa} ; e_{15}=e_{24}=$ $12.322 \mathrm{Cm}^{-2} ; e_{31}=e_{32}=-7.209 \mathrm{Cm}^{-2} ; e_{33}=15.118 \mathrm{Cm}^{-2} ; \epsilon_{11}^{\sigma}=\epsilon_{22}^{\sigma}=15.3 \mathrm{nFm}^{-1}$; $\epsilon_{33}^{\sigma}=15 n \mathrm{Fm}^{-1} ; \rho_{p z t}=7500 \mathrm{~kg} \cdot \mathrm{m}^{-3}$. Para o Graphite - Epoxy, as propriedades utilizadas foram: $c_{11}=183.443 \mathrm{GPa} ; c_{22}=c_{33}=11.662 \mathrm{GPa} ; c_{12}=c_{13}=4.363 \mathrm{GPa}$; $c_{23}=3.918 \mathrm{GPa} ; c_{44}=2.870 \mathrm{GPa} ; c_{55}=c_{66}=2.870 \mathrm{GPa} ; \rho_{G E}=1580 \mathrm{kgm}^{-3}$. As condições de contorno utilizadas neste estudo foram para a placa apoiada em duas bordas paralelas mantendo-se livre as outras bordas (SSFF - Simply Suported e Free).

A tabela 2.6 apresenta uma comparação das frequencias em circuito aberto (OC) entre o modelo proposto por Baillargeon e Vel [7] e o modelo desenvolvido neste trabalho, para a estrutura acima descrita.

$\mathrm{Na}$ tabela abaixo apresentamos os resultados em circuito fechado (SC) para a estrutura descrita utilizando o modelo proposto por Baillargeon e Vel [7] e o modelo proposto. 


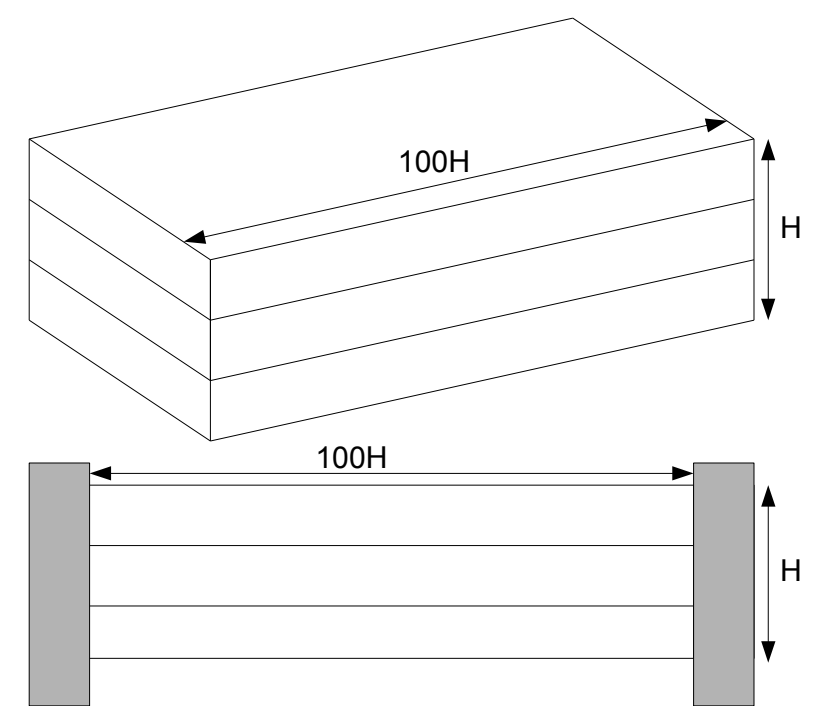

Figura 2.5: Viga sanduíche "infinita"biapoiada com camada piezelétrica em cisalhamento

Tabela 2.6: Tabela com os resultados obtidos para comparação de placa em circutio aberto.

\begin{tabular}{|c|c|c|c|}
\hline & Modelo Placa & Referência [7] & Diferença \\
\hline & Frequencia em OC $(\mathrm{Hz})$ & Frequencia em OC $(\mathrm{Hz})$ & $\%$ \\
\hline$f_{1,1}$ & 145.21 & 145.06 & +0.10 \\
\hline$f_{2,1}$ & 580.72 & 578.65 & +0.35 \\
\hline$f_{3,1}$ & 1306.09 & 1296.04 & +0.77 \\
\hline$f_{4,1}$ & 2320.63 & 2289.53 & +1.35 \\
\hline$f_{5,1}$ & 3623.37 & 3548.85 & +2.09 \\
\hline$f_{6,1}$ & 5213.07 & 5061.46 & +2.99 \\
\hline$f_{7,1}$ & 7088.23 & 6813.07 & +4.03 \\
\hline$f_{8,1}$ & 9247.10 & 8788.09 & +5.22 \\
\hline
\end{tabular}

Tabela 2.7: Tabela com os resultados obtidos para comparação de placa em circutio fechado.

\begin{tabular}{|c|c|c|c|}
\hline & Modelo Placa & Referência [7] & Diferença \\
\hline & Frequencia em SC $(\mathrm{Hz})$ & Freqüência em SC $(\mathrm{Hz})$ & $\%$ \\
\hline$f_{1,1}$ & 145.21 & 145.06 & +0.10 \\
\hline$f_{2,1}$ & 580.72 & 578.54 & +0.37 \\
\hline$f_{3,1}$ & 1306.09 & 1295.49 & +0.81 \\
\hline$f_{4,1}$ & 2320.63 & 2287.84 & +1.4 \\
\hline$f_{5,1}$ & 3623.37 & 3544.82 & +2.21 \\
\hline$f_{6,1}$ & 5213.07 & 5053.35 & +3.15 \\
\hline$f_{7,1}$ & 7088.23 & 6798.55 & +4.26 \\
\hline$f_{8,1}$ & 9247.10 & 8764.26 & +5.50 \\
\hline
\end{tabular}




\subsection{Conclusão}

Os resultados de comparação entre frequências naturais obtidas com o modelo proposto e aquelas encontradas na literatura apresentados neste capítulo indicam que o modelo proposto é adequado para uso na análise dinâmica de placas sanduíche com camadas piezelétricas. Embora, algumas diferenças superiores a 5\% tenham sido encontradas em alguns casos, ressalta-se que, face à dificuldade em se encontrar resultados comparáveis na literatura, foram utilizados exemplos que não são totalmente apropriados para tratamento com o modelo proposto (estruturas tipo viga ou estruturas muito espessas). É esperado que para configurações mais próximas daquela de interesse, isto é placas sanduíche com camadas relativamente finas ( $\mathrm{L} / \mathrm{H}$ entre 25 e 75 ), os resultados obtidos pelo modelo proposto sejam melhores. 


\section{Capítulo 3}

\section{Acoplamento dos circuitos elétricos nas}

\section{estruturas piezoelétricas}

Até agora foram considerados somente os efeitos elétricos devido as propriedades piezoelétricas do material, porém é necessário analisar o acoplamento do circuito elétrico na estrutura. Os circuitos elétricos, devem ser representados em função de carga elétrica, pois será este o vínculo com a carga elétrica gerada nas camadas piezoelétricas. Neste trabalho, serão considerados apenas circuitos RLV em série.

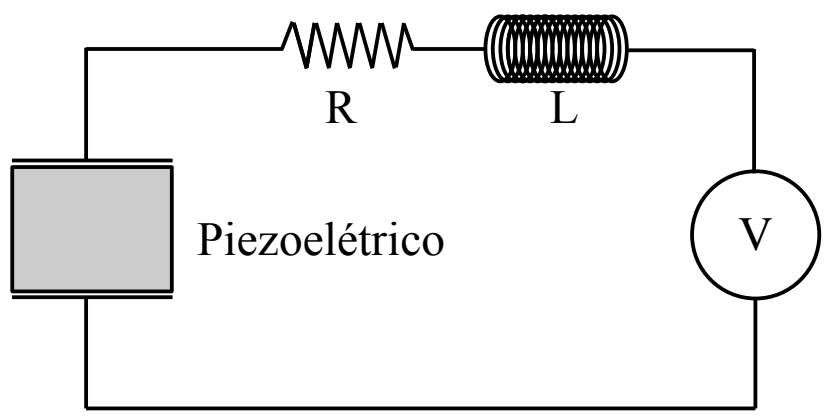

Figura 3.1: Configuração de um circuito elétrico com resistência (R), indutor (L), fonte de tensão $(V)$ e elemento piezoelétrico 


\subsection{Relação entre cargas elétricas nos circuitos e nos ele- mentos piezoelétricos}

Levando em conta a conexão com o circuito elétrico, é proposto que as cargas elétricas que entram no circuito elétrico sejam iguais às cargas elétricas geradas nos eletrodos de cada camada piezoelétrica. Esta relação pode ser escrita como,

$$
\mathbf{q}_{c}=\mathbf{L}_{q} \mathbf{q}_{p}
$$

onde $\mathbf{L}_{q}$ é uma matriz binária. Esta matriz binária é a responsável por especificar se $\mathrm{o}$ circuito está ou não conectado à estrutura e a que eletrodo, mais especificamente. Devido a equipotencialidade nos eletrodos que revestem as superfícies de cada camada piezoelétrica, o deslocamento elétrico que surgirá ao longo do eletrodo será constante e, portanto, as cargas elétricas para cada elemento piezoelétrico são obtidas multiplicando-se o deslocamento elétrico pela área do eletrodo que reveste este elemento. Assim, definindose uma matriz diagonal $\mathbf{A}_{q}$ cujos elementos representam a área de cada elemento piezoelétrico eletrodo definido. Então, o vetor de cargas elétricas em cada eletrodo pode ser escrito como

$$
\mathbf{q}_{p}=\mathbf{A}_{q} \mathbf{D}_{p}
$$

Conseqüentemente, a entrada das cargas elétricas nos $n$ circuitos possíveis, podem ser escritas em termos do deslocamento elétrico do elemento piezoelétrico como

$$
\mathbf{q}_{c}=\mathbf{B}_{q} \mathbf{D}_{p} ; \quad \mathbf{B}_{q}=\mathbf{L}_{q} \mathbf{A}_{q}
$$




\subsection{Equações de movimento para o sistema acoplado}

Para acoplar a dinâmica da estrutura piezoelétrica com aquela dos circuitos elétricos conectados a ela, primeiramente encontra-se os trabalhos virtuais realizados pelos elementos do circuito e, em seguida, estes são inseridos no princípio variacional.

\section{Trabalho virtual realizado pelos componentes do circuito elétrico}

O trabalho virtual realizado pelos componentes do circuito elétrico pode ser escrito em função de cada um dos seus componentes separadamente, pois cada componente do circuito corresponde a um trabalho virtual. Então tem-se o trabalho virtual realizado pelas indutâncias, $\delta W_{c j}^{c}$, resistências, $\delta W_{r j}^{e x}$, e fontes de potência, $\delta W_{e j}^{e x}$, do $j$-ésimo circuito elétrico,

$$
\delta W_{c j}^{c}=-\delta q_{c j} L_{c j} \ddot{q}_{c j} ; \delta W_{r j}^{e x}=-\delta q_{c j} R_{c j} \dot{q}_{c j} ; \delta W_{\varphi j}^{e x}=\delta q_{c j} \varphi_{c j}
$$

onde $L_{c j}, R_{c j}$ e $\varphi_{c j}$ são respectivamente a indutância, a resistência e a tensão elétrica aplicada no $j$-ésimo circuito elétrico. $q_{c j}$ é a carga elétrica total do $j$-ésimo circuito elétrico. Usando uma forma matricial, pode-se reescrever (3.4) como

$$
\begin{aligned}
& \delta W_{c j}^{c}=\sum_{j=1}^{n} \delta W_{c j}^{c}=-\delta \mathbf{q}_{c}^{t} \mathbf{L}_{c} \ddot{\mathbf{q}}_{c} \\
& \delta W_{r j}^{e x}=\sum_{j=1}^{n} \delta W_{r j}^{e x}=-\delta \mathbf{q}_{c} \mathbf{R}_{c} \dot{\mathbf{q}}_{c} \\
& \delta W_{\varphi j}^{e x}=\sum_{j=1}^{n} \delta W_{\varphi j}^{e x}=\delta \mathbf{q}_{c} \boldsymbol{\varphi}_{c}
\end{aligned}
$$

sendo $\mathbf{q}_{c}$ um vetor contendo as cargas elétricas em cada circuito, $\mathbf{L}_{c}$ e $\mathbf{R}_{c}$ matrizes diagonais contendo as indutâncias e resistências de cada circuito e $\boldsymbol{\varphi}_{c}$ um vetor contendo as tensões elétricas aplicadas a cada circuito. 


\subsubsection{Equações do movimento}

Aos trabalhos virtuais considerados na equação (2.18), somam-se agora os trabalhos virtuais realizados pelos elementos dos circuitos elétricos considerados. Tem-se então

$$
\delta W^{c}+\delta W^{i n}+\delta W_{e x}+\delta W_{c}^{c}+\delta W_{r}^{e x}+\delta W_{\varphi}^{e x}=0
$$

Usando a forma variacional da equação (2.80), tem-se então

$$
\delta \mathbf{u}^{t}\left(\mathbf{M} \ddot{u}+\mathbf{K}_{m} u-\overline{\mathbf{K}}_{m e} \mathbf{D}_{p}-\mathbf{F}\right)+\delta \mathbf{D}_{p}^{t}\left(-\overline{\mathbf{K}}_{m e}^{t} u+\overline{\mathbf{K}}_{e} \mathbf{D}_{p}\right)+\delta \mathbf{q}_{c}^{t}\left(\mathbf{L}_{c} \ddot{\mathbf{q}}_{c}+\mathbf{R}_{c} \dot{\mathbf{q}}_{c}-\varphi_{c}\right)=0
$$

Considerando-se a equação (3.3) para escrever $\mathbf{q}_{c}$ em função de $\mathbf{D}_{p}$ obtém-se as equações de movimento para o sistema acoplado

$$
\left[\begin{array}{cc}
\mathbf{M} & 0 \\
0 & \mathbf{M}_{q}
\end{array}\right]\left\{\begin{array}{c}
\ddot{\mathbf{u}} \\
\ddot{\mathbf{D}}_{p}
\end{array}\right\}+\left[\begin{array}{cc}
0 & 0 \\
0 & \mathbf{C}_{q}
\end{array}\right]\left\{\begin{array}{c}
\dot{\mathbf{u}} \\
\dot{\mathbf{D}}_{p}
\end{array}\right\}+\left[\begin{array}{cc}
\mathbf{K}_{m} & -\overline{\mathbf{K}}_{m e} \\
-\overline{\mathbf{K}}_{m e}^{t} & \overline{\mathbf{K}}_{e}
\end{array}\right]\left\{\begin{array}{c}
\mathbf{u} \\
\mathbf{D}_{p}
\end{array}\right\}=\left\{\begin{array}{c}
0 \\
\mathbf{F}_{q}
\end{array}\right\}
$$

sendo

$$
\mathbf{M}_{q}=\mathbf{B}_{q}^{t} \mathbf{L}_{c} \mathbf{B}_{q} ; \mathbf{C}_{q}=\mathbf{B}_{q}^{t} \mathbf{R}_{c} \mathbf{B}_{q} ; \mathbf{F}_{q}=\mathbf{B}_{q}^{t} \varphi_{c}
$$

Vale a pena ressaltar que no caso da equação (3.8), a solução para os graus de liberdade mecânicos e elétricos deve ser simultânea.

Nesta formulação, a aplicação de uma tensão elétrica a um atuador piezoelétrico se dá de forma indireta, isto é, a tensão elétrica gera uma carga elétrica no circuito correspondente a qual alimenta o eletrodo do atuador. 


\subsection{Análise Harmônica}

Nesta seção, faz-se uma análise harmônica para obter uma avaliação preliminar dos efeitos causados pelos componentes elétricos do circuito na estrutura. Para isso considera-se dois casos: uma excitação harmônica elétrica (tensão elétrica aplicada ao circuito) e uma excitação harmônica mecânica (força aplicada à estrutura). Em ambos os casos, um único elemento piezoelétrico conectado a um circuito de RLV é considerado.

\subsubsection{Excitação puramente mecânica}

Supondo uma excitação mecânica harmônica, tal que

$$
\varphi_{c}=0 ; \mathbf{F}=\mathbf{b} \tilde{f} e^{i \omega t} ; \mathbf{u}=\tilde{\mathbf{u}} e^{i \omega t} ; \mathbf{D}_{p}=\tilde{\mathbf{D}}_{p} e^{i \omega t}
$$

Então, as equações de movimento (3.8) poderam ser reescritos como sendo,

$$
\begin{aligned}
& \left(-\omega^{2} M+K_{m}\right) \tilde{\mathbf{u}}-\bar{K}_{m e} \tilde{\mathbf{D}}_{p}=\mathbf{b} \tilde{f} \\
& -\bar{K}_{m e}^{t} \tilde{\mathbf{u}}+\left(-\omega^{2} M_{q}+i \omega C_{q}+\bar{K}_{e}\right) \tilde{\mathbf{D}}_{p}=0
\end{aligned}
$$

Resolvendo a segunda equação de (3.11) para $\tilde{\mathbf{D}}_{p}$ e substituindo na primeira equação tem-se,

$$
\left\{\left(-\omega^{2} M+K_{m}-\bar{K}_{m e}\left(-\omega^{2} M_{q}+i \omega C_{q}+\bar{K}_{e}\right)^{-1} \bar{K}_{m e}^{t}\right)\right\} \tilde{\mathbf{u}}=\mathbf{b} \tilde{f}
$$

Supondo a medição do deslocamento de um ponto selecionado da estrutura tal que

$$
\tilde{y}=\mathbf{c}_{p} \tilde{\mathbf{u}}
$$

onde $\tilde{y}$ é o sinal de deslocamento medido e $\mathbf{c}_{p}$ é um vetor de distribuição de saída. Então, a função de resposta em freqüência do deslocamento da estrutura sujeita a uma força aplicada pode ser escrita a partir da relação. 


$$
\tilde{y}=G(\omega) \tilde{f}
$$

sendo

$$
G_{p}(\omega)=\mathbf{c}_{p}\left\{\left(-\omega^{2} \mathbf{M}+\mathbf{K}_{m}-\overline{\mathbf{K}}_{m e}\left(-\omega^{2} \mathbf{M}_{q}+i \omega \mathbf{C}_{q}+\overline{\mathbf{K}}_{e}\right)^{-1} \overline{\mathbf{K}}_{m e}^{t}\right)\right\}^{-1} \mathbf{b}
$$

Através da expressão de $G(\omega)$, pode-se notar que a resistência e a indutância têm o efeito de modificar a rigidez dinâmica da estrutura. Em particular, dois casos simplificados podem ser observados: quando $\mathbf{C}_{q} \rightarrow \infty$, representando um abertura do circuito, não há modificação na rigidez da estrutura, tal que

$$
G_{p}(\omega)=\mathbf{c}_{p}\left\{-\omega^{2} \mathbf{M}+\mathbf{K}_{m}\right\}^{-1} \mathbf{b}
$$

enquanto que quando $\mathbf{M}_{q}=\mathbf{C}_{q}=0$, representando um curto circuito a rigidez da estrutura é reduzida, mas continua real, tal que

$$
G_{p}(\omega)=\mathbf{c}_{p}\left\{-\omega^{2} \mathbf{M}+\left[\mathbf{K}_{m}-\overline{\mathbf{K}}_{m e} \bar{K}_{e}^{-1} \overline{\mathbf{K}}_{m e}^{t}\right]\right\}^{-1} \mathbf{b}
$$

A modificação da rigidez da estrutura, dada pelo termo $\left[\mathbf{K}_{m}-\overline{\mathbf{K}}_{m e} \bar{K}_{e}^{-1} \overline{\mathbf{K}}_{m e}^{t}\right]$ é devida à correção da constante elástica do material piezoelétrico para a condição de campo elétrico constante $\left(\mathbf{c}_{i j}^{E}\right)$ por oposição à de deslocamento elétrico constante $\left(\mathbf{c}_{i j}^{D}\right)$.

\subsubsection{Excitação puramente elétrica}

Supondo uma excitação elétrica harmônica, tal que

$$
\mathbf{F}=0 ; \varphi_{c}=\tilde{\varphi}_{c} e^{i \omega t} ; \mathbf{u}=\tilde{\mathbf{u}} e^{i \omega t} ; \mathbf{D}_{p}=\tilde{\mathbf{D}}_{p} e^{i \omega t}
$$

Então, as equações de movimento 3.8 podem ser reescritas como sendo, 


$$
\begin{aligned}
& \left(-\omega^{2} \mathbf{M}+\mathbf{K}_{m}\right) \tilde{\mathbf{u}}-\overline{\mathbf{K}}_{m e} \tilde{\mathbf{D}}_{p}=0 \\
& -\overline{\mathbf{K}}_{m e}^{t} \tilde{\mathbf{u}}+\left(-\omega^{2} \mathbf{M}_{q}+i \omega \mathbf{C}_{q}+\overline{\mathbf{K}}_{e}\right) \tilde{\mathbf{D}}_{p}=\mathbf{B}_{q}^{t} \tilde{\varphi}_{c}
\end{aligned}
$$

Resolvendo a segunda equação de (3.19) para $\tilde{\mathbf{D}}_{p}$ e substituindo na primeira equação tem-se

$$
\begin{array}{r}
\left\{-\omega^{2} \mathbf{M}+\left[\mathbf{K}_{m}-\overline{\mathbf{K}}_{m e}\left(-\omega^{2} \mathbf{M}_{q}+i \omega \mathbf{C}_{q}+\overline{\mathbf{K}}_{e}\right)^{-1} \overline{\mathbf{K}}_{m e}^{t}\right]\right\} \tilde{\mathbf{u}}= \\
\overline{\mathbf{K}}_{m e}\left(-\omega^{2} \mathbf{M}_{q}+i \omega \mathbf{C}_{q}+\overline{\mathbf{K}}_{e}\right)^{-1} \mathbf{B}_{q}^{t} \tilde{\varphi}_{c}
\end{array}
$$

Supondo a medição do deslocamento de um ponto selecionado da estrutura tal que

$$
\tilde{y}=\mathbf{c}_{p} \tilde{\mathbf{u}}
$$

onde $\tilde{y}$ é sinal de deslocamento medido e $\mathbf{c}_{p}$ é um vetor de distribuição de saída. Então, a função de resposta em freqüência do deslocamento da estrutura sujeita a uma tensão elétrica aplicada pode ser escrita a partir da relação

$$
\tilde{y}=G(\omega) \tilde{\varphi}_{c}
$$

sendo

$$
\begin{array}{r}
G_{c}(\omega)=\mathbf{c}_{p}\left\{-\omega^{2} \mathbf{M}+\left[\mathbf{K}_{m}-\overline{\mathbf{K}}_{m e}\left(-\omega^{2} \mathbf{M}_{q}+i \omega \mathbf{C}_{q}+\overline{\mathbf{K}}_{e}\right)^{-1} \overline{\mathbf{K}}_{m e}^{t}\right]\right\}^{-1} \times \\
\overline{\mathbf{K}}_{m e}\left(-\omega^{2} \mathbf{M}_{q}+i \omega \mathbf{C}_{q}+\overline{\mathbf{K}}_{e}\right)^{-1} \mathbf{B}_{q}^{t}
\end{array}
$$

Através da expressão para $G(\omega)$, pode-se notar que a resistência e as indutâncias do circuito elétrico têm dois efeitos. O primeiro efeito é uma modificação passiva na rigidez dinâmica da estrutura. Em particular, nota-se que no caso de um circuito sem resistência ou indutância, ou seja, com $\mathbf{M}_{q}=\mathbf{C}_{q}=0$, 


$$
G_{c}(\omega)=\mathbf{c}_{p}\left\{-\omega^{2} \mathbf{M}+\left[\mathbf{K}_{m}-\overline{\mathbf{K}}_{m e} \bar{K}_{e}^{-1} \overline{\mathbf{K}}_{m e}^{t}\right]\right\}^{-1} \overline{\mathbf{K}}_{m e} \overline{\mathbf{K}}_{e}^{-1} \mathbf{B}_{q}^{t}
$$

Este caso representa a aplicação de uma tensão elétrica diretamente no elemento piezoelétrico, fazendo com que ele se comporte como um atuador simples, tal que o termo $\left[\overline{\mathbf{K}}_{m e} \overline{\mathbf{K}}_{e}^{-1} \mathbf{B}_{q}^{t}\right]$ representa a força por unidade de tensão elétrica aplicada à estrutura.

Uma simulação de circuito aberto pode ser realizada fazendo $\mathbf{C}_{q} \rightarrow \infty$, ou seja, uma alta resistência. Neste caso, a rigidez da estrutura não é alterada, mas a atuação da estrutura se torna impossível.

No caso mais geral, a presença da resistência no circuito elétrico leva a valores complexos na matriz de rigidez que pode ser interpretada com a introdução de um amortecimento histerético na estrutura. A presença da indutância dá origem a uma ressonância elétrica, que pode ser utilizada para induzir uma absorção das vibrações estruturais, como será visto no próximo capítulo.

\subsection{Projeto e análise de controle passivo de vibrações}

Os componentes elétricos passivos, resistência e indutância, nos circuitos elétricos conectados aos elementos piezoelétricos podem ser ajustados de forma a modificar o comportamento dinâmico do circuito e, consequentemente, alterar o comportamento dinâmico da estrutura. Conforme observado no capítulo anterior, a inclusão de uma resistência no circuito pode dar origem a um amortecimento histerético na estrutura, uma vez que a rigidez global do sistema estrutura/piezoelétrico/circuito se torna complexa. Este efeito pode ser explicado pela dissipação de energia elétrica na resistência, via efeito Joule, sendo que esta energia é gerada pela deformação do material piezoelétrico e, conseqüientemente, pela vibração da estrutura. Assim, parte da energia vibratória da estrutura é convertida em energia elétrica pelo material piezoelétrico e parte desta energia elétrica é dissipada na resistência. Por outro lado, a inclusão de uma indutância no circuito dá origem a uma 
frequência de ressonância para o circuito. Este fato pode ser utilizado em benefício da redução das vibrações da estrutura ajustando a frequência de ressonância do circuito de modo a absorver uma parcela da energia vibratória da estrutura. Este é o conceito de absorvedor dinâmico de vibrações. Assim, neste capítulo pretende-se usar a metodologia de projeto de absorvedores dinâmicos de vibração para ajustar as propriedades dos subsistemas, circuitos elétricos, considerados como absorvedores. Em seguida, uma análise de seu desempenho é realizada para dois exemplos.

\subsection{Projeto do absorvedor dinâmico de vibrações}

Considere a equação de movimento da estrutura piezoelétrica com circuitos elétricos passivos (RL) acoplados,

$$
\begin{aligned}
& \mathbf{M u ̈}+\mathbf{K}_{m} \mathbf{u}-\overline{\mathbf{K}}_{m e} \mathbf{D}_{p}=\mathbf{F}=\mathbf{b} f \\
& \mathbf{M}_{q} \ddot{\mathbf{D}}_{p}+\mathbf{C}_{q} \dot{\mathbf{D}}_{p}+\overline{\mathbf{K}}_{e} \mathbf{D}_{p}-\overline{\mathbf{K}}_{m e} \mathbf{u}=0 \\
& y=\mathbf{c}_{p} \mathbf{u}
\end{aligned}
$$

Por simplicidade, as vibrações da estrutura são aproximadas pela contribuição de um único modo de vibração, cuja frequência de ressonância seja a frequência para a qual se deseja projetar o absorvedor dinâmico. Assim, uma decomposição modal do deslocamento u é considerada

$$
\mathbf{u}(t)=\boldsymbol{\phi}_{n} \alpha_{n}(t)
$$

sendo $n$ referente à frequência de ressonância de interesse, $\boldsymbol{\phi}_{n}$ o modo de vibração correspondente e $\alpha_{n}$ o deslocamento modal.

Multiplicando a primeira equação de (3.25) por $\boldsymbol{\phi}_{n}^{t}$, substituindo o deslocamento $\mathbf{u}$ (3.26) e supondo um único par pastilha piezoelétrica-circuito elétrico, pode-se reescrever a equação (3.25) como 


$$
\begin{aligned}
& \ddot{\alpha}_{n}+\omega_{n}^{2} \alpha_{n}-k_{p} D_{p}=b_{n} f \\
& m_{q} \ddot{D}_{p}+c_{q} \dot{D}_{p}+k_{e} D_{p}-k_{p} \alpha_{n}=0 \\
& y=c_{n} \alpha_{n}
\end{aligned}
$$

sendo que

$$
\begin{aligned}
& \boldsymbol{\phi}_{n}{ }^{t} \mathbf{M} \boldsymbol{\phi}_{n}=1 \\
& \boldsymbol{\phi}_{n}{ }^{t} \mathbf{K}_{m} \boldsymbol{\phi}_{n}=\omega_{n}{ }^{2} \\
& \boldsymbol{\phi}_{n}{ }^{t} \mathbf{K}_{m e}=k_{p} \\
& b_{n}=\boldsymbol{\phi}_{n}^{t} \mathbf{b} \\
& c_{n}=c \boldsymbol{\phi}_{n}
\end{aligned}
$$

e supondo uma excitação mecânica harmônica do sistema, tal que

$$
\begin{aligned}
& f=\bar{f} e^{i \omega t} \\
& \alpha_{n}=\bar{\alpha}_{n} e^{i \omega t} \\
& D_{p}=\bar{D}_{p} e^{i \omega t} \\
& y=\bar{y} e^{i \omega t}
\end{aligned}
$$

Pode-se reescrever a equação (3.27) como

$$
\begin{aligned}
& \left(-\omega^{2}+\omega_{n}^{2}\right) \bar{\alpha}_{n}-k_{p} \bar{D}_{p}=b_{n} \bar{f} \\
& \left(-\omega^{2} m_{q}+i \omega c_{q}+k_{e}\right) \bar{D}_{p}-k_{p} \bar{\alpha}_{n}=0 \\
& \bar{y}=c_{n} \bar{\alpha}_{n}
\end{aligned}
$$

Resolvendo a segunda equação de (3.30) para o deslocamento elétrico, tem-se

$$
\bar{D}_{p}=\frac{k_{p}}{\left(-\omega^{2} m_{q}+i \omega c_{q}+k_{e}\right)} \bar{\alpha}_{n}
$$

Em seguida, substituindo-se o deslocamento elétrico (3.31) na primeira equação de (3.30) 
obtém-se

$$
\left[\left(-\omega^{2}+\omega_{n}^{2}\right)-\frac{k_{p}^{2}}{\left(-\omega^{2} m_{q}+i \omega c_{q}+k_{e}\right)}\right] \bar{\alpha}_{n}=b_{n} \bar{f}
$$

tal que a amplitude do deslocamento modal $\bar{\alpha}_{n}$ pode ser escrita em função da amplitude de forçamento $\bar{f}$ por

$$
\bar{\alpha}_{n}=\frac{\left(-\omega^{2} m_{q}+i \omega c_{q}+k_{e}\right)}{\left(-\omega^{2}+\omega_{n}^{2}\right)\left(-\omega^{2} m_{q}+i \omega c_{q}+k_{e}\right)-k_{p}^{2}} b_{n} \bar{f}
$$

Assim, a amplitude do deslocamento de saída $\bar{y}$, usando a terceira equação de (3.30), pode ser escrita em função da amplitude de forçamento, tal que a função de resposta em frequência complexa entre $\bar{y}$ e $\bar{f}$, tal que $\bar{y}=G(\omega) \bar{f}$, pode ser definida como

$$
G(\omega)=\frac{c_{n}\left(-\omega^{2} m_{q}+i \omega c_{q}+k_{e}\right) b_{n}}{\omega^{4} m_{q}-i \omega^{3} c_{q}-\omega^{2} k_{e}-\omega^{2} m_{q} \omega_{n}^{2}+i \omega \omega_{n}^{2} c_{q}+k_{e} \omega_{n}^{2}-k_{p}^{2}}
$$

Para obter a amplitude de $G(\omega)$, reescreve-se a equação (3.34) da seguinte forma

$$
G(\omega)=r_{n}\left(\frac{A+i B}{C+i D}\right)
$$

sendo

$$
\begin{aligned}
& r_{n}=c_{n} b_{n} \\
& A=-\omega^{2} m_{q}+k_{e} \\
& B=\omega c_{q} \\
& C=\omega^{4} m_{q}-\omega^{2}\left(k_{e}+m_{q} \omega_{n}^{2}\right)+k_{e} \omega_{n}^{2}-k_{p}^{2} \\
& D=\left(-\omega^{2}+\omega_{n}^{2}\right) \omega c_{q}
\end{aligned}
$$

Assim, o quadrado da amplitude de $G(\omega)$ pode ser escrito como 


$$
|G(\omega)|^{2}=r_{n}^{2}\left(\frac{A^{2}+B^{2}}{C^{2}+D^{2}}\right)
$$

ou

$$
|G(\omega)|^{2}=r_{n}^{2} \frac{\left(-\omega^{2} m_{q}+k_{e}\right)^{2}+\left(\omega c_{q}\right)^{2}}{\left[\omega^{4} m_{q}-\omega^{2}\left(k_{e}+m_{q} \omega_{n}^{2}\right)+k_{e} \omega_{n}^{2}-k_{p}^{2}\right]^{2}+\left[\left(-\omega^{2}+\omega_{n}^{2}\right) \omega c_{q}\right]^{2}}
$$

Pode-se então utilizar a amplitude da função de resposta em frequência para projetar os parâmetros do circuito, $m_{q}\left(L_{c}\right)$ e $c_{q}\left(R_{c}\right)$. Uma estratégia comumente utilizada é projetar, a frequência de ressonância do sistema auxiliar, no caso o circuito elétrico, de forma que uma anti-ressonância no sistema acoplado seja obtida na frequência de ressonância do sistema principal, no caso a estrutura piezoelétrica [25, 20].

Desta maneira, e visto que a rigidez dielétrica do material piezoelétrico $k_{e}$ é tida como arbitrária, a inércia equivalente do circuito $m_{q}$ pode ser definida de tal maneira que a frequência de ressonância do circuito $\omega_{e}=\sqrt{k_{e} / m_{q}}$ seja igual à frequência de ressonância $\omega_{n}$ da estrutura, em torno da qual se deseje minimizar a amplitude de vibração. Assim, $m_{q}=k_{e} / \omega_{n}^{2}$.

Contudo, o efeito secundário de se posicionar uma anti-ressonância na frequência de interesse é que duas ressonâncias, logo antes e logo depois da anti-ressonância, são criadas. Pode-se então projetar o outro parâmetro do circuito elétrico, a resistência, para minimizar a amplitude nas ressonâncias sem para tanto eliminar a redução da amplitude na frequência de interesse dada pela anti-ressonância. Uma técnica para projetar este parâmetro de amortecimento foi proposta por Den Hartog [20] e será utilizada neste trabalho.

Para tal, observa-se que a amplitude da resposta em frequência é independente do amortecimento para dois valores de frequência, os quais se encontram nos limites da região de ressonância, ou seja, um é menor e o outro é maior que ambas as frequências de 
ressonância.

Estes valores de frequência podem ser encontradas da seguinte forma

$$
\lim _{c_{q} \rightarrow 0}|G(\omega)|^{2}=\lim _{c_{q} \rightarrow \infty}|G(\omega)|^{2}
$$

sendo que

$$
\begin{gathered}
\lim _{c_{q} \rightarrow 0}|G(\omega)|^{2}=r_{n}^{2} \frac{\left(k_{e}-m_{q} \omega^{2}\right)^{2}}{\left[\left(\omega^{4} m_{q}-\omega^{2}\left(k_{e}+m_{q} \omega_{n}^{2}\right)+k_{e} \omega_{n}^{2}-k_{p}^{2}\right)\right]^{2}} \\
\lim _{c_{q} \rightarrow \infty}|G(\omega)|^{2}=r_{n}^{2} \frac{1}{\left(\omega^{2}-\omega_{n}^{2}\right)^{2}}
\end{gathered}
$$

Assim,

$$
\frac{k_{e}-m_{q} \omega^{2}}{\omega^{4} m_{q}-\omega^{2}\left(k_{e}+m_{q} \omega_{n}^{2}\right)+k_{e} \omega_{n}^{2}-k_{p}^{2}}=\frac{1}{\left(\omega^{2}-\omega_{n}^{2}\right)}
$$

da qual obtemos uma equação de segunda ordem, em $\omega^{2}$

$$
2 m_{q} \omega^{4}+2 \omega^{2}\left(k_{e}+m_{q} \omega_{n}^{2}\right)-2 k_{e} \omega_{n}^{2}+k_{p}^{2}=0
$$

Resolvendo a equação (3.43) encontramos dois valores para $\omega^{2}$,

$$
\omega_{1,2}^{2}=\frac{1}{2}\left(\omega_{e}^{2}+\omega_{n}^{2} \pm \sqrt{\left(\omega_{e}^{2}-\omega_{n}^{2}\right)^{2}+2 \omega_{e} \frac{k_{p}^{2}}{k_{e}}}\right)
$$

Primeiramente, pode-se observar que

$$
\omega_{1}^{2}+\omega_{2}^{2}=\omega_{e}^{2}+\omega_{n}^{2}
$$

Assim, mostra-se que o projeto de $m_{q}$ tal que $\omega_{e}=\omega_{n}$ garante uma mesma amplitude nos valores de frequência $\omega_{1}$ e $\omega_{2}$ 


$$
\frac{1}{\omega_{1}^{2}-\omega_{n}^{2}}=-\frac{1}{\omega_{2}^{2}-\omega_{n}^{2}}
$$

da qual

$$
\omega_{1}^{2}+\omega_{2}^{2}-2 \omega_{n}^{2}=0
$$

e substituindo a equação (3.45) tem-se,

$$
\omega_{e}=\omega_{n}
$$

Em seguida, com o valor de $m_{q}$ definido, projeta-se o amortecimento equivalente $c_{q}$ tal que a amplitude na anti-ressonância do sistema acoplado $\left(\omega_{n}=\omega_{e}\right)$ seja igual àquela nas frequências $\omega_{1}$ e $\omega_{2}$, de forma a limitar a amplitude nas ressonâncias do sistema acoplado.

Assim, calculando-se a amplitude na equação (3.38) para as frequências $\omega_{n}$ e $\omega_{1}$ (3.44), tem-se

$$
\begin{aligned}
\left|G\left(\omega_{n}\right)\right|^{2} & =\frac{2 k_{e}}{k_{p}^{2} \omega_{n}^{2}} \\
\left|G\left(\omega_{1}\right)\right|^{2} & =\frac{c_{q}{ }^{2} \omega_{n}{ }^{2}}{k_{p}{ }^{4}}
\end{aligned}
$$

Igualando as amplitudes em $\omega_{n}$ e $\omega_{1}$ e resolvendo esta equação para o amortecimento equivalente $c_{q}$, obtém-se o seguinte valor de projeto para $c_{q}$ em função da rigidez eletromecânica e da frequência de ressonância de interesse

$$
c_{q}=\frac{\sqrt{2 k_{e}} k_{p}}{\omega_{n}^{2}}
$$

Com base nas equações (3.46) e (3.50) e lembrando que a inércia, $m_{q}$, e amortecimento, $c_{q}$, equivalentes do circuito elétrico podem ser escritas em função da indutância, $L_{c}$, e resistência, $R_{c}$, tal que $m_{q}=L_{c} A_{p}^{2}$ e $c_{q}=R_{c} A_{p}^{2}$ e $A_{p}$ é a área do eletrodo da pastilha 
piezoelétrica, tem-se as fórmulas para projeto dos componentes passivos do circuito, $R_{c} \mathrm{e}$ $L_{c}$

$$
\begin{gathered}
L_{c}=\frac{k_{e}}{A_{p}^{2} \omega_{n}^{2}} \\
R_{c}=\frac{\sqrt{2 k_{e}} k_{p}}{A_{p}^{2} \omega_{n}^{2}}
\end{gathered}
$$




\subsection{Projeto da lei de controle}

Para prover um controle ativo das vibrações estruturais, uma lei de controle é necessária para se calcular a tensão elétrica a ser aplicada no circuito. O algoritmo de controle considerado foi uma versão interativa do controle ótimo LQR (Linear Quadratic Regulator) conforme apresentada em [61]. Esta lei de controle de retroalimentação foi considerada para o desenvolvimento da parte ativa do controle para o modo em questão. Para isto foi necessário reescrever as equações de movimento (3.8) na forma do espaço de estado com um vetor de estado das variaveis $\mathbf{z}$ é definido, contendo os deslocamentos modais e as velocidades dos modos de vibrações de interesse e os deslocamentos elétricos dos elementos piezoelétricos a suas derivadas. Isto nos leva a escrever,

$$
\dot{\mathbf{z}}=\hat{\mathbf{A}} \mathbf{z}+\hat{\mathbf{B}} \mathbf{V}_{c}+\hat{\mathbf{B}}_{f} \mathbf{f}, \mathbf{y}=\hat{\mathbf{C}}_{y} \mathbf{z}
$$

onde

$$
\begin{aligned}
\mathbf{z} & =\left[\begin{array}{c}
\alpha \\
\mathbf{D}_{p} \\
\dot{\alpha} \\
\mathbf{D}_{p}
\end{array}\right], \hat{\mathbf{A}}=\left[\begin{array}{cccc}
\mathbf{0} & \mathbf{0} & \mathbf{I} & \mathbf{0} \\
\mathbf{0} & \mathbf{0} & \mathbf{0} & \mathbf{I} \\
-\boldsymbol{\Omega}^{2} & \mathbf{K}_{p} & -\Lambda & \mathbf{0} \\
\mathbf{M}_{q}^{-1} \mathbf{K}_{p}^{t} & -\boldsymbol{\Omega}_{e}^{2} & \mathbf{0} & -\boldsymbol{\Lambda}_{e}
\end{array}\right], \\
\hat{\mathbf{B}} & =\left[\begin{array}{c}
\mathbf{0} \\
\mathbf{0} \\
\mathbf{0} \\
\mathbf{M}_{q}^{-1} \mathbf{A}_{p}^{t}
\end{array}\right], \hat{\mathbf{B}}_{f}=\left[\begin{array}{c}
\mathbf{0} \\
\mathbf{0} \\
\mathbf{b}_{\phi} \\
\mathbf{0}
\end{array}\right], \hat{\mathbf{C}}_{y}=\left[\begin{array}{llll}
\mathbf{c}_{\phi} & \mathbf{0} & \mathbf{0} & \mathbf{0}
\end{array}\right] .
\end{aligned}
$$

Os deslocamentos modais são definidos por $\mathbf{u}=\boldsymbol{\Phi} \boldsymbol{\alpha}$ e, para a massa normalizada dos modos de vibrações, $\Omega^{2}=\Phi^{t} \mathbf{K}_{m} \Phi$ e $\Lambda=\Phi^{t} \mathbf{C} \Phi$. $\Omega$ é a matriz diagonal onde os elementos são as frequências naturais não amortecidas da estrutura com o material piezoelétrico em circuito aberto. $\Omega_{e}^{2}=\mathbf{M}_{q}^{-1} \overline{\mathbf{K}}_{e}$ e $\Lambda_{e}=\mathbf{M}_{q}^{-1} \mathbf{C}_{q}$ são ambos matrizes diagonais cujos elementos estão, respectivamente, para as frequencia naturais ao quadrado 
do circuito elétrico e a relação entre resistências e indutâncias $\left(\mathbf{M}_{q}^{-1} \mathbf{C}_{q}=\mathbf{L}_{c}^{-1} \mathbf{R}_{c}\right)$. A matriz de rigidez do acoplamento eletromecânico projetado na base modal não amortecida é definido como $\mathbf{K}_{p}=\Phi^{t} \overline{\mathbf{K}}_{m e}$. Os vetores de entrada $\mathbf{b}$ e saida $\mathbf{c}$ são definidos com as projeções modais $\mathbf{b}_{\phi}=\Phi^{t} \mathbf{b}$ e $\mathbf{c}_{\phi}=\mathbf{c} \Phi$, e $\mathbf{f}$ é um vetor de amplitudes de cada força aplicada sobre a estrutura.

Um estado de retroalimentação linear para a voltagem $\mathbf{V}_{c}$ foi assumido onde $\mathbf{V}_{c}=$ $-\mathbf{g z}=-\mathbf{g}_{d m} \boldsymbol{\alpha}-\mathbf{g}_{d e} \mathbf{D}_{p}-\mathbf{g}_{v m} \dot{\boldsymbol{\alpha}}-\mathbf{g}_{v e} \dot{\mathbf{D}}_{p}$, onde $\mathbf{g}$ é um vetor de ganhos para o controle de cada variavél de estado. Dessa forma, a equação de espaço de estado (3.53) se torna,

$$
\dot{\mathbf{z}}=(\hat{\mathbf{A}}-\hat{\mathbf{B}} \mathbf{g}) \mathbf{z}+\hat{\mathbf{B}}_{f} \mathbf{f}, \mathbf{y}=\hat{\mathbf{C}}_{y} \mathbf{z}
$$

Para uma única exitação mecânica $f$, o controle fechado ou controle da amplitude de um único deslocamento com saida $y$ pode ser escrito como sendo $\tilde{y}=G_{h}(\omega) \tilde{f}$, onde a FRF $G_{h}(\omega)$ é

$$
G_{h}(\omega)=\hat{\mathbf{C}}_{y}(\mathbf{j} \omega \mathbf{I}-\hat{\mathbf{A}}+\hat{\mathbf{B} g})^{-1} \hat{\mathbf{B}}_{f}
$$

que também pode ser derivada a partir das equações de segunda ordem do movimento com projeção na base modal não-amortecida levando a

$$
\begin{aligned}
& G_{h}(\omega)=\mathbf{c}_{\phi}\left\{-\omega^{2} \mathbf{I}+\mathrm{j} \omega\left(\boldsymbol{\Lambda}+\mathbf{K}_{p} \mathbf{D}_{c c}^{-1} \mathbf{A}_{p}^{\mathrm{t}} \mathbf{g}_{v m}\right)\right. \\
&\left.+\left[\boldsymbol{\Omega}^{2}+\mathbf{K}_{p} \mathbf{D}_{c c}^{-1}\left(\mathbf{A}_{p}^{\mathrm{t}} \mathbf{g}_{d m}-\mathbf{K}_{p}^{\mathrm{t}}\right)\right]\right\}^{-1} \mathbf{b}_{\phi}
\end{aligned}
$$

onde a rigidez dinâmica de malha fechada do circuito elétrico $\mathbf{D}_{c c}$ é

$$
\mathbf{D}_{c c}=-\omega^{2} \mathbf{M}_{q}+\mathrm{j} \omega\left(\mathbf{C}_{q}+\mathbf{A}_{p}^{\mathrm{t}} \mathbf{g}_{v e}\right)+\left(\overline{\mathbf{K}}_{e}+\mathbf{A}_{p}^{\mathrm{t}} \mathbf{g}_{d e}\right) .
$$

Neste trabalho, o ganho do controle $\mathbf{g}$ é calculado usando um padrão ótimo que é 
calculado usando a teoria padrão de controle ótimo LQR, aplicado a um caso de única entrada e única saida, pois a estrutura está apenas com um elemento ativo-passivo para o controle minimizar a amplitude de vibração em um local específico da estrutura. A seguinte função objetivo é minimizada,

$$
J=\frac{1}{2} \int_{0}^{\infty}\left(\dot{y}^{2}+r V_{c}^{2}\right) \mathrm{d} t
$$

onde $\dot{y}$ é a velocidade do local de interesse e $V_{c}$ é a voltagem de controle aplicada no circuito ativo-passive. $\mathrm{O}$ fator de ponderação $r$ é automaticamente ajustado para garantir o máximo controle sobre a voltagem de $200 \mathrm{~V}$ em todos os casos, seguindo a rotina proposta em [61].

\subsection{Resultados para o controle ativo-passivo}

Nesta seção, apresenta-se resultados de controle passivo para estruturas em configurações de cerâmicas piezoelétricas em extensão com circuitos passivos resistivos e resistivoindutivos. Será realizada uma análise da resposta em frequiência para os cinco primeiros modos de vibração sendo que os valores dos componentes dos circuitos elétricos serão ajustados para acoplamento com o primeiro modo de vibração da estrutura. Uma análise das alterações resultantes na resposta na região freqüências de ressonâncias é apresentada.

\subsubsection{Estrutura com cerâmica piezoelétrica em extensão}

A análise de uma viga engastada será realizada através da resposta em frequência para pastilhas piezoelétricas em extensão conforme a Figura 2.3 que apresenta a configuração considerada.

As propriedades dos materiais considerados nesta configuração foram as mesmas utilizadas no Capítulo 2.5. 


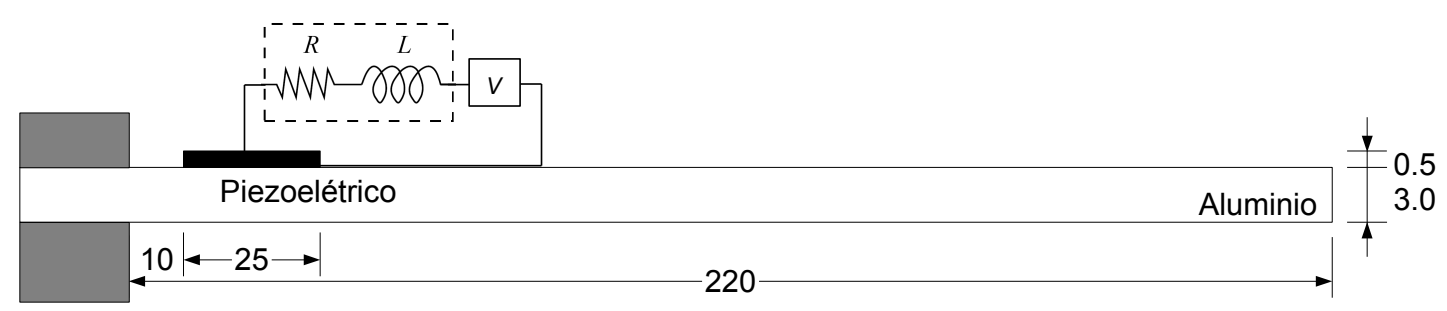

Figura 3.2: Configuração da estrutura piezoelétrica em extensão

Foram considerados os cinco primeiro modos de vibração da estrutura. Utilizando as equações (3.51) e (3.52) da seção anterior, realizou-se o cálculo dos valores dos componentes elétricos $R$ e $L$ utilizados no circuito elétrico RL. Para o circuito RL, como visto no capítulo anterior, os valores dos componentes foram ajustados para o primeiro modo de vibração e dando origem a resistência $R=2,117710^{4} \Omega$ e indutância $L=377,6830 \mathrm{H}$, estes valores foram ajustados em 1,01\% para a resistência e 1,02\% para a indutânica. Este ajuste foi realizado de tal maneira a alinhar os dois picos criados pelo circuito elétrico.

Conforme pode ser observado na Figura 3.3, no caso do circuito resistivo-indutivo, pode-se notar na Figura que a ressonância da estrutura é substituída por duas ressonâncias acopladas com uma anti-ressonância entre elas. Conforme especificado no projeto do absorvedor dinâmico equivalente, a anti-ressonância é posicionada na frequência de ressonância da estrutura de base de forma que a amplitude nesta frequência é reduzida em aproximadamente $20 \mathrm{~dB}$ em comparação com a estrutura de base (sem tratamento).

Observa-se na Figura 3.3 que a amplitude da ressonância, para o caso onde o circuito RL está conectado a uma fonte de tensão, apresentou uma redução de $25 \mathrm{~dB}$ em comparação com a estrutura de base (sem o tratamento), não alterando a forma da ressonância.

Na Figura 3.3, observa-se que a amplitude nas ressonâncias geradas é igual, devido ao ajuste realizado manualmente, onde este pequeno erro pode indicar imprecisão no ajuste da frequência de anti-ressonância. Acredita-se que este fato se deva à não consideração do amortecimento estrutural de $0.5 \%$ no projeto dos componentes do circuito. 


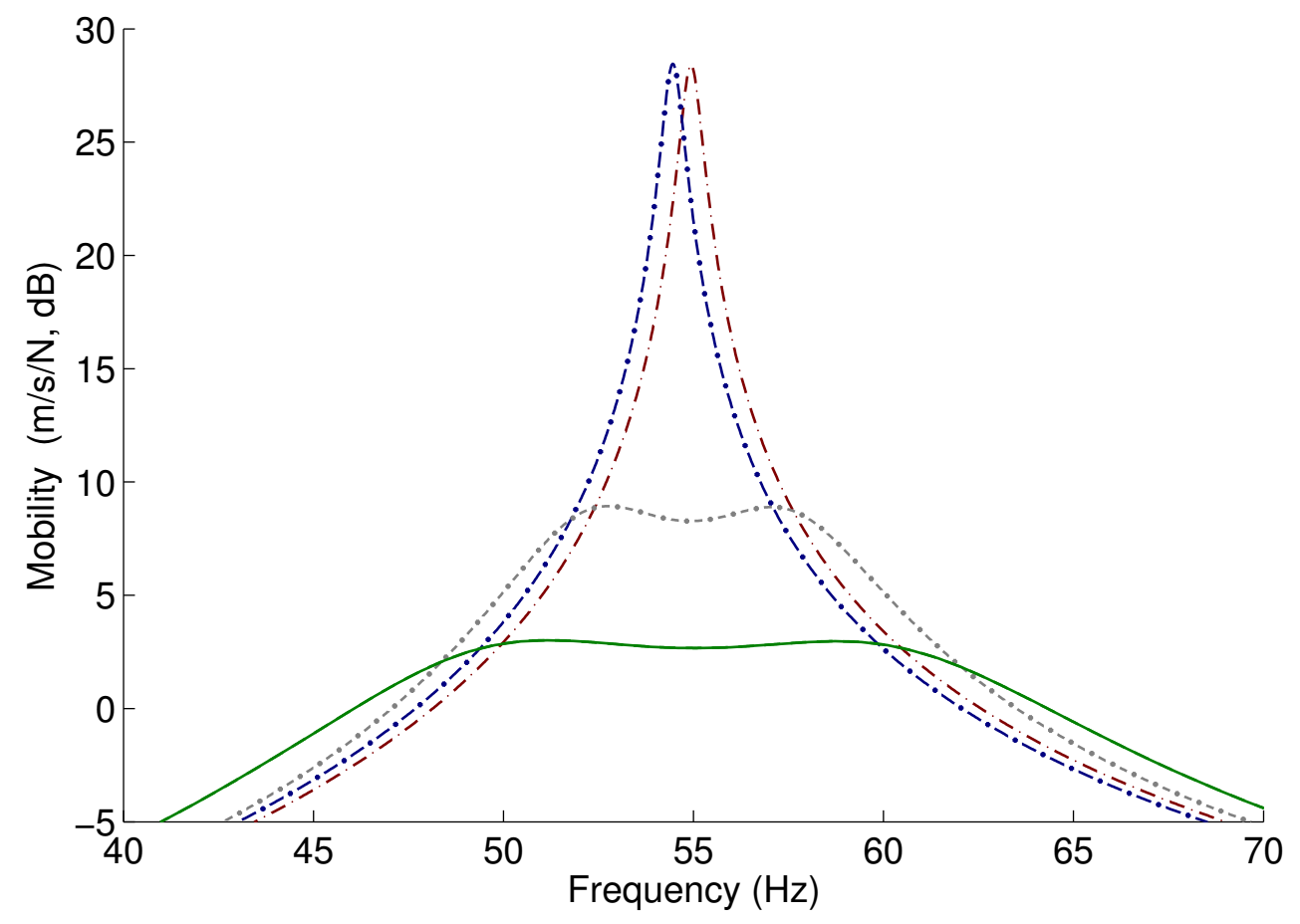

Figura 3.3: Primeira ressonância da viga com piezocerâmica em extensão: circuito aberto (traço longo ponto-pequeno); curto-circuito (traço longo ponto); circuito RL (traço curto ponto); circuito RLV (traço continuo) 


\section{Capítulo 4}

\section{Modelagem estocástica para análise de}

\section{incertezas}

Esta seção apresenta uma abordagem para a análise de incertezas para algumas variáveis na modelagem em estruturas piezelétricas. Neste trabalho consideraremos incertezas na constante dielétrica $\beta_{33}^{\mathcal{\varepsilon}}$, acoplamento piezelétrico $\bar{h}_{31}$ e $\bar{h}_{32}$, ambas propriedades inerentes ao material piezelétrico utilizado e para a resistência $R_{c}$ e indutância $L_{c}$, ambos elementos do circuito elétrico dissipativo. Para simplificar a descrição da metodologia adotada para as 4 variáveis utilizadas para realizar esta análise, faremos $X=\left[\beta_{33}^{\varepsilon}, \bar{h}_{31}, \bar{h}_{32}, R_{c}, L_{c}\right]$. Desta forma um modelo estocástico para cada variável em $X$ é construído levando-se em conta as informações disponíveis para cada uma das variáveis de tal maneira que: 1) a variável estocástica deve estar entre $] 0,+\infty[;$ (2) a média dos valores é tal que $E[X]=\underline{X}$; e (3) o zero é um valor descartado para variáveis com valores positivos, que é explicado pela condição $E[\ln (X)]=c_{X} \operatorname{com}\left|c_{X}\right|<+\infty$. Desta maneira, o Princípio de Máxima Entropia, produz uma função densidade de probabilidade Gamma [45, 40, 37, 16].

$$
p_{X}(X)=\mathbb{I}_{0,+\infty}(X) \frac{1}{\underline{X}}\left(\frac{1}{\delta_{X}^{2}}\right)^{\delta_{X}^{-2}} \frac{1}{\Gamma\left(\delta_{X}^{-2}\right)}\left(\frac{X}{\underline{X}}\right)^{\delta_{X}^{-2}-1} \exp \left(-\frac{X}{\delta_{X}^{2} \underline{X}}\right)
$$

observamos que $\delta_{X}=\sigma_{X} / \underline{X}$ é a dispersão relativa de $\widehat{X}$ e $\sigma_{X}$ é o desvio padrão. A 

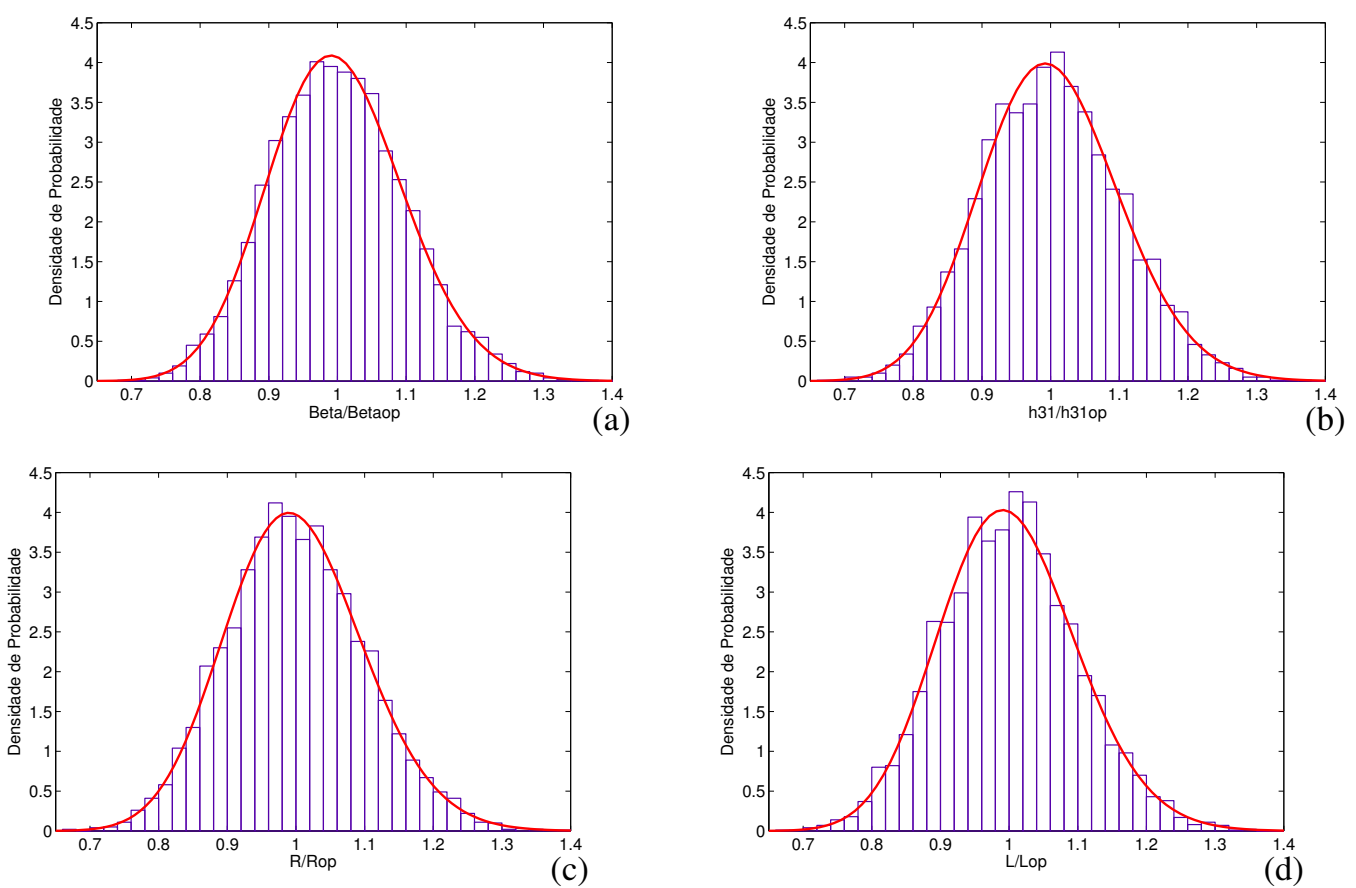

Figura 4.1: Função de densidade para distribuição Gamma e histogramas das propriedades do material a) $\bar{\beta}_{33}^{\varepsilon}$, b) $\bar{h}_{31}$ e para parâmetros do circuito c) $R_{c}$ e d) $L_{c}$.

função Gamma é definida como $\Gamma(\alpha)=\int_{0}^{\infty} t^{\alpha-1} e^{-t} \mathrm{~d} t$. Para uma análise de cada uma das variáveis podemos observar a função densidade de probabilidade (Probability Density Functions, (p.d.f.)) e o histograma das variáveis aleatórias. Como descrito as variáveis foram obtidas utilizando uma função MATLAB, gamrnd, considerando 5000 realizações e podemos observar que ela segue o comportamento esperado de uma função Gamma, somente com valores positivos e sem picos fora da distribuição. Estes vetores com as realizações aleatórias para a variável $\widehat{X}$, serão combinadas em conjunto de 5 variáveis, que serão aplicadas nas propriedades de entrada do modelo desenvolvido e assim gerar uma nova FRF $G_{p}\left(\theta_{j}, \omega\right), G_{c}\left(\theta_{j}, \omega\right)$ e $G_{h}\left(\theta_{j}, \omega\right)$.

Uma análise de convergência com as realizações utilizando o conjunto de variáveis independentes, $\widehat{G}_{p}(\omega)$, pode ser obtido utilizando a função,

$$
\operatorname{conv}\left(n_{s}\right)=\frac{1}{n_{s}} \sum_{j=1}^{n_{s}} \int\left\|G_{p}\left(\theta_{j}, \omega\right)-G_{p}^{N}(\omega)\right\|^{2} \omega
$$



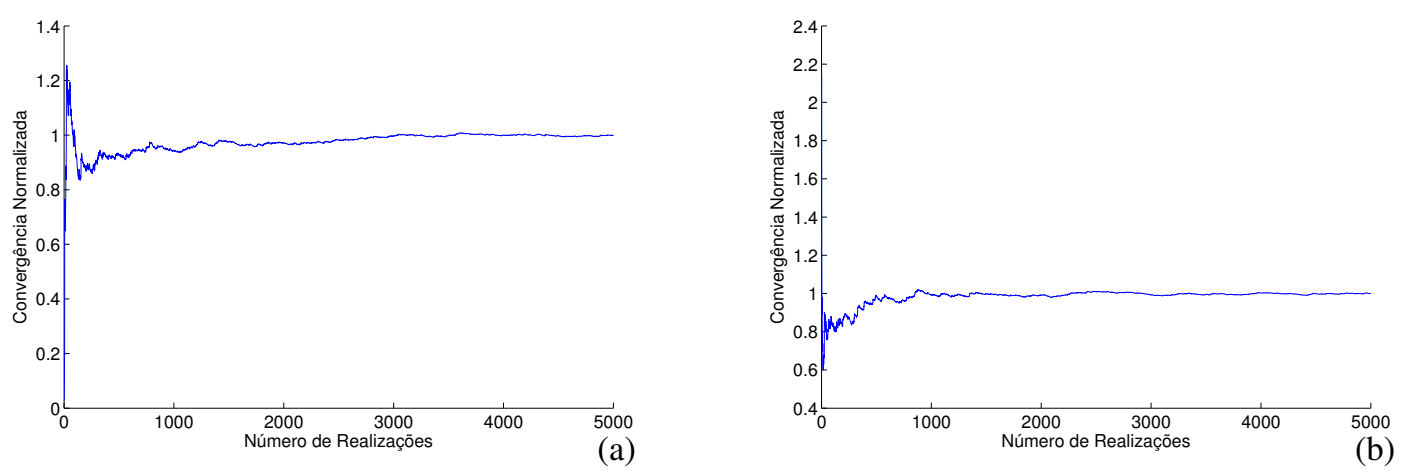

Figura 4.2: Convergencia das simulações realizadas através do método de Monte Carlos considerando incerteza nos parametros: (a) somente $L_{c}$ e (b) $\bar{\beta} \bar{\varepsilon}, \overline{h_{31}}, \overline{h_{32}}, R_{c}$ e $L_{c}$

onde $n_{s}$ é o numero de simulações e $G_{p}^{N}(\omega)$ é a resposta calculada para o modelo médio correspondente. Através da Figura 4.2 podemos observar que a média quadrática da convergência para a configuração em extensão considerando um $\delta_{X}=0.10$. É possível observar que para todos os casos 3000 realizações são suficientes para assegurar a convergência dos resultados. As análises estatísticas apresentadas a seguir consideraram 5000 realizações.

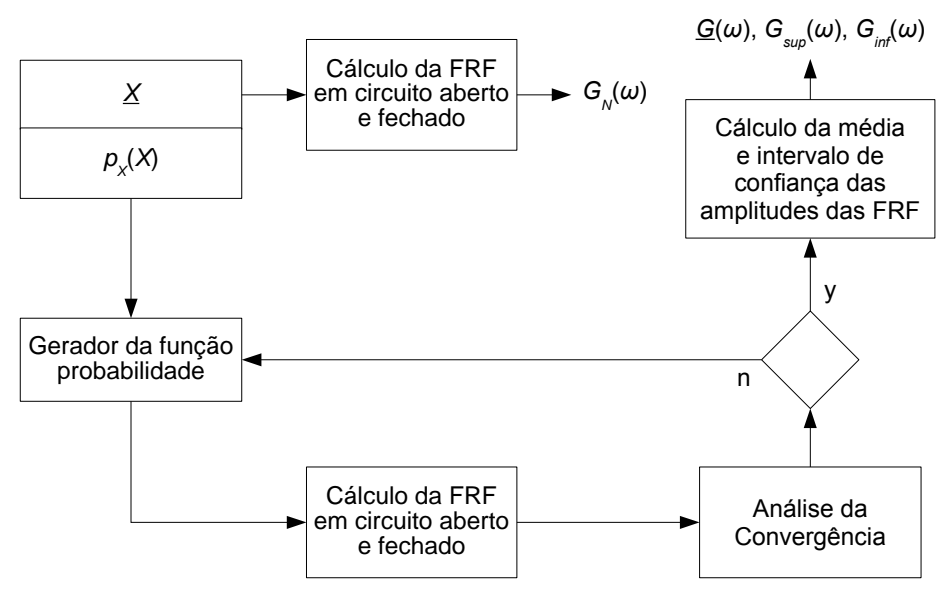

Figura 4.3: Esquema do procedimento computacional para obtenção das respostas em frequencia e o calculo do intervalo de confiança.

As análises das amplitudes das respostas em frequências (FRF), como descrito acima, foram realizadas utilizando as 5000 realizações para cada valor de frequência, 
calculando o valor médio e o intervalo de confiança de $95 \%$. Este processo pode ser observado em detalhes na Figura 6.2.

\subsection{Análise dos resultados obtidos}

Nesta seção ilustramos os resultados obtidos através das realizações para uma estrutura com as dimensões de uma viga com $220 \mathrm{~mm}$ de comprimento com $25 \mathrm{~mm}$ de largura sendo o elemento piezelétrico acoplado em sua superfície, localizado a $10 \mathrm{~mm}$ do seu engaste. Neste caso, a viga considerada tem somente uma de suas extremidades engastada. As cerâmicas piezelétricas são feitas de PZT-5H e as propriedades consideradas do material piezelétrico foram: $\bar{c}_{11}^{D}=\bar{c}_{22}^{D}=97.767 \mathrm{GPa}, \bar{c}_{12}^{D}=50.774 \mathrm{GPa}, \bar{c}_{44}^{D}=\bar{c}_{55}^{D}=42.217 \mathrm{GPa}$, $\rho=7500 \mathrm{~kg} \mathrm{~m}^{-3}$, constante de acoplamento piezelétrica $\bar{h}_{31}=-1.352010^{9} \mathrm{~N} \mathrm{C}^{-1} \mathrm{e}$ constante dielétrica $\bar{\beta}_{33}^{\varepsilon}=57.83010^{6} \mathrm{~m} \mathrm{~F}^{-1}$. Para a viga de alumínio, as propriedades do material são definidas por: Módulo de Young $70.3 \mathrm{GPa}$ e densidade $2710 \mathrm{~kg} \mathrm{~m}^{-3}$, um amortecimento viscoso de $0.5 \%$ foi considerado. Para os componentes do circuito elétrico foram considerados os valores de resistência de $R_{c}=21177 \Omega$ e para a indutância de $L_{c}=377.68 H$.

Foi realizada uma análise do efeito individual de cada uma das variáveis, no comportamento da estrutura, possibilitando assim uma análise mais precisa da influência destas perturbações na resposta da estrutura considerada.

\subsubsection{Análise da influência de incertezas para propriedades dielétricas do material $\left(\bar{\beta}_{33}^{\varepsilon}\right)$}

Uma análise realizada considerando a configuração em extensão da estrutura proposta acima pode ser vista na figura 4.4, onde a resposta em frequência em circuito aberto (OC) $\left(G_{p}^{O C}\right)$ e conectada a um circuito shunt otimizado $\left(G_{p}^{N}\right)$ podem ser observada, lembrando que foram utilizadas as 5000 realizações variando somente a propriedade piezelétrica para 
gerar o intervalo de confiança.

Analisando as respostas para as incertezas na propriedade o intervalo de confiança mostrou uma redução de 28 para $10 \mathrm{~dB}$ com o valor médio e de 28 para $17 \mathrm{~dB}$ para o limite superior. Realizou-se uma análise da influência do controle neste caso, onde observou-se que, como apresentado pela figura 4.4(b) com ganho constante, a redução entre a estrutura em circuito aberto e o limite superior foi de $20.2 \mathrm{~dB}$. No caso apresentado pela figura 4.4(c) com ganho variável, a redução entre a estrutura em circuito aberto e o limite superior foi de $23.3 \mathrm{~dB}$. Desta meneira reduzindo a dispersão e consequentemente aumentando a robustez.
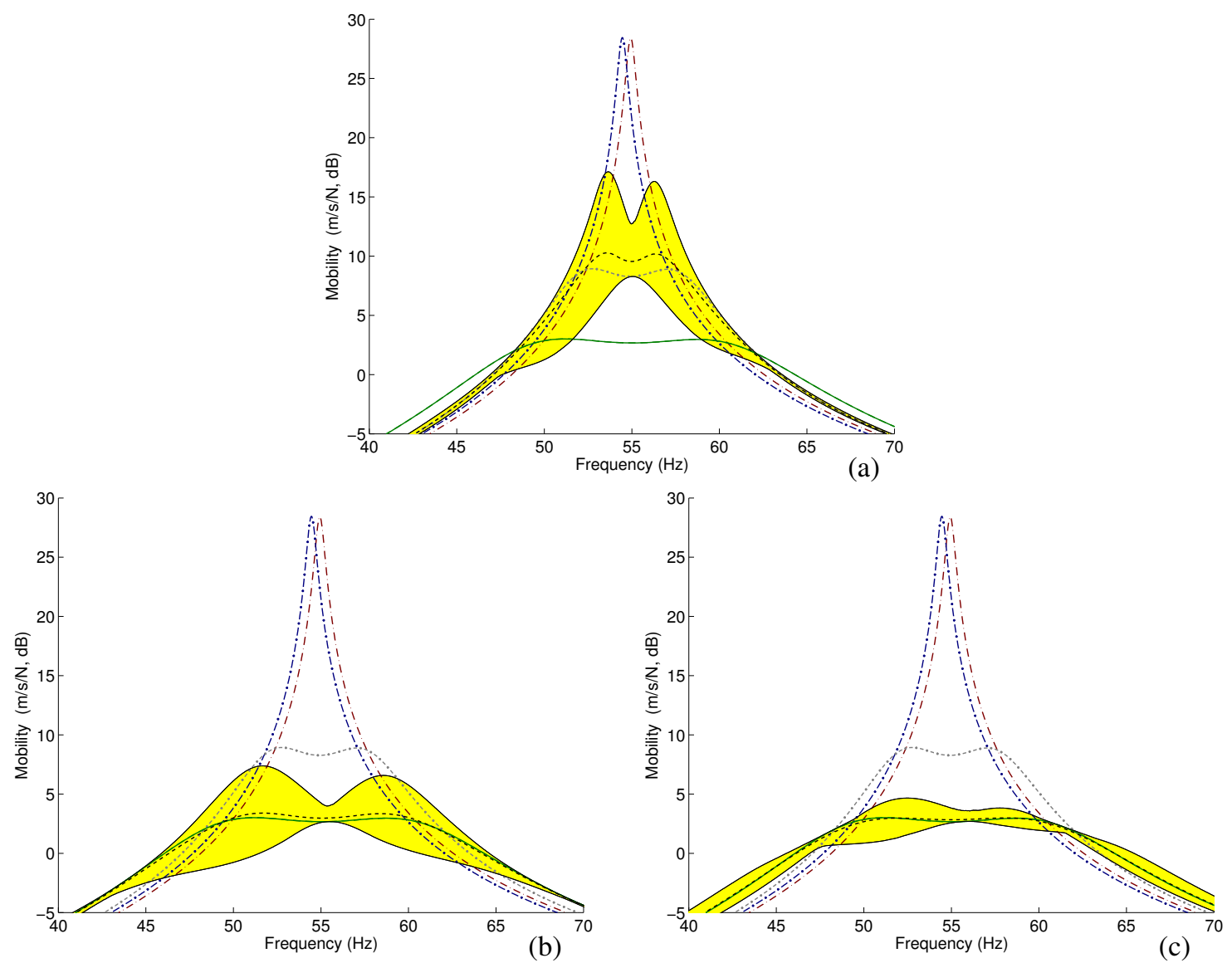

Figura 4.4: Análise estocástica para o primeiro modo com incerteza na propriedade do material $\bar{\beta}_{33}^{\varepsilon}$ (a) Passivo Puro, (b) Ativo-Passivo com ganho constante e (c) Ativo-Passivo com ganho variável. 


\subsubsection{Análise da influência de incertezas para propriedades pie- zelétricas do material $\left(h_{31}\right)$}

Uma análise realizada considerando a configuração em extensão da estrutura proposta acima pode ser vista na figura 4.5, onde a resposta em frequência em circuito aberto (OC) $\left(G_{p}^{O C}\right)$ e conectada a um circuito shunt otimizado $\left(G_{p}^{N}\right)$ pode ser observada, lembrando que foram utilizadas as 5000 realizações variando somente as propriedades piezelétricas $h_{31}$ para gerar o intervalo de confiança.

Analisando as respostas para as incertezas nas propriedades $\left(h_{31}\right)$ o intervalo de confiança mostrou uma redução de 28 para $8.5 \mathrm{~dB}$ com o valor médio e de 28 para $15.5 \mathrm{~dB}$ para o limite superior. Realizou-se uma análise da influência do controle neste caso, onde observou-se que, como apresentado pela figura 4.5(b) com ganho constante, a redução entre a estrutura em circuito aberto e o limite superior foi de $21 \mathrm{~dB}$. No caso apresentado pela figura 4.5(c) com ganho variável, a redução entre a estrutura em circuito aberto e o limite superior foi de $22.2 \mathrm{~dB}$.

\subsubsection{Análise da influência de incertezas para as propriedades resis- tivas do circuito elétrico $R_{C}$}

Uma análise realizada considerando a configuração em extensão da estrutura proposta acima pode ser vista na figura 4.6, onde a resposta em frequência em circuito aberto (OC)

$\left(G_{p}^{O C}\right)$ e conectada a um circuito shunt otimizado $\left(G_{p}^{N}\right)$ pode ser observada, lembrando que foram utilizadas as 5000 realizações variando somente a propriedade do circuito elétrico conectado $R_{c}$ para gerar o intervalo de confiança. Analisando as respostas para as incertezas para o componente do circuito shunt $\left(R_{c}\right)$ o intervalo de confiança mostrou uma redução de 28 para $8 \mathrm{~dB}$ com o valor médio e de 28 para $9.5 \mathrm{~dB}$ para o limite superior.

Realizou-se uma análise da influência do controle neste caso, onde observou-se que, como apresentado pela figura 4.6(b) com ganho constante, a redução entre a estrutura em 

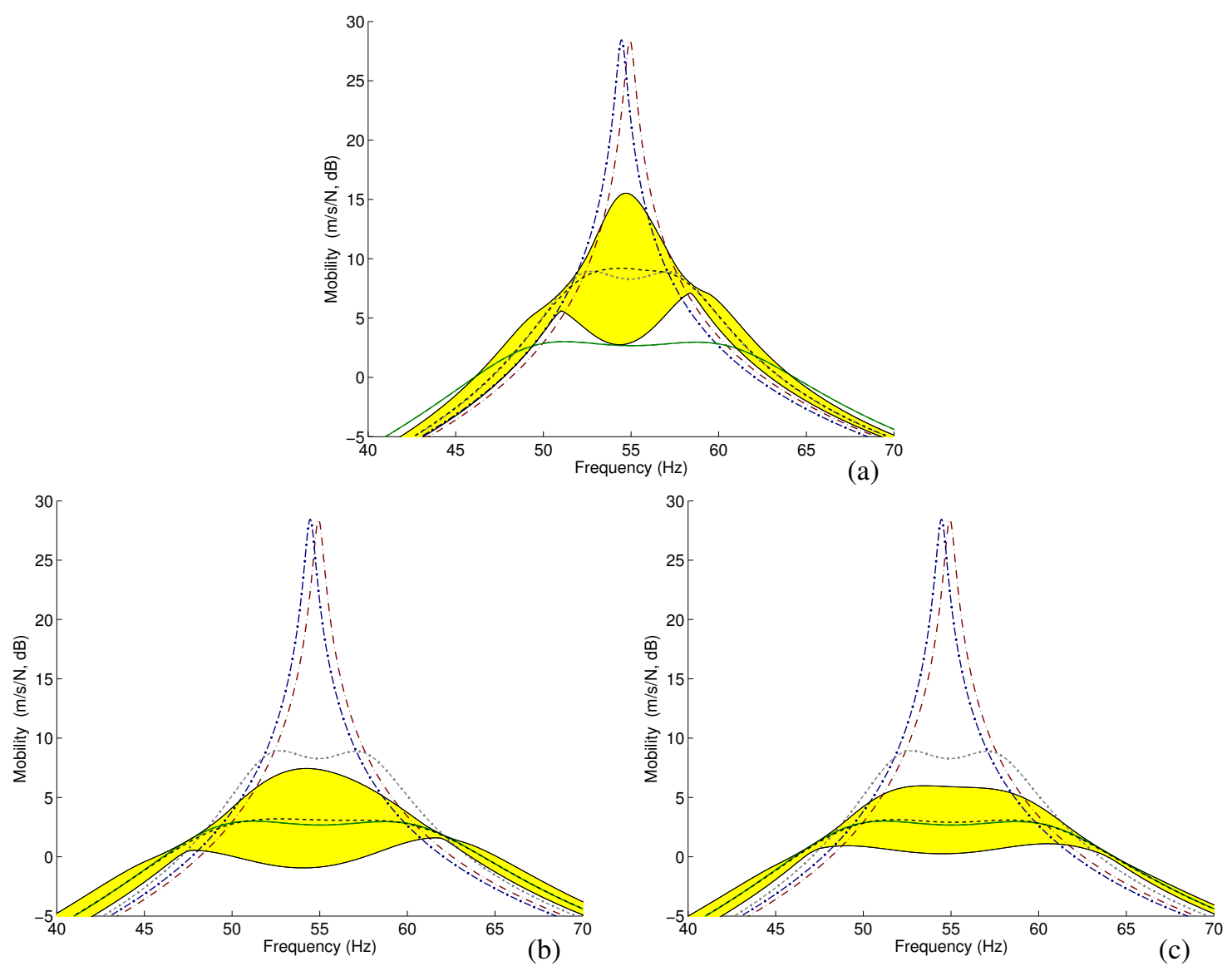

Figura 4.5: Análise estocástica para o primeiro modo com incerteza nas propriedades do material $h_{31}$ (a) Passivo Puro, (b) Ativo-Passivo com ganho constante e (c) Ativo-Passivo com ganho variável.

circuito aberto e o limite superior foi de $24 \mathrm{~dB}$. No caso apresentado pela figura 4.6(c) com ganho variável, a redução entre a estrutura em circuito aberto e o limite superior foi de $24.8 \mathrm{~dB}$.

\subsubsection{Análise da influência de incertezas para propriedades indutivas do circuito elétrico $L_{c}$}

Uma análise realizada considerando a configuração em extensão da estrutura proposta acima pode ser vista na figura 4.7, onde a resposta em frequência em circuito aberto (OC) $\left(G_{p}^{O C}\right)$ e conectada a um circuito shunt otimizado $\left(G_{p}^{N}\right)$ pode ser observada, lembrando 

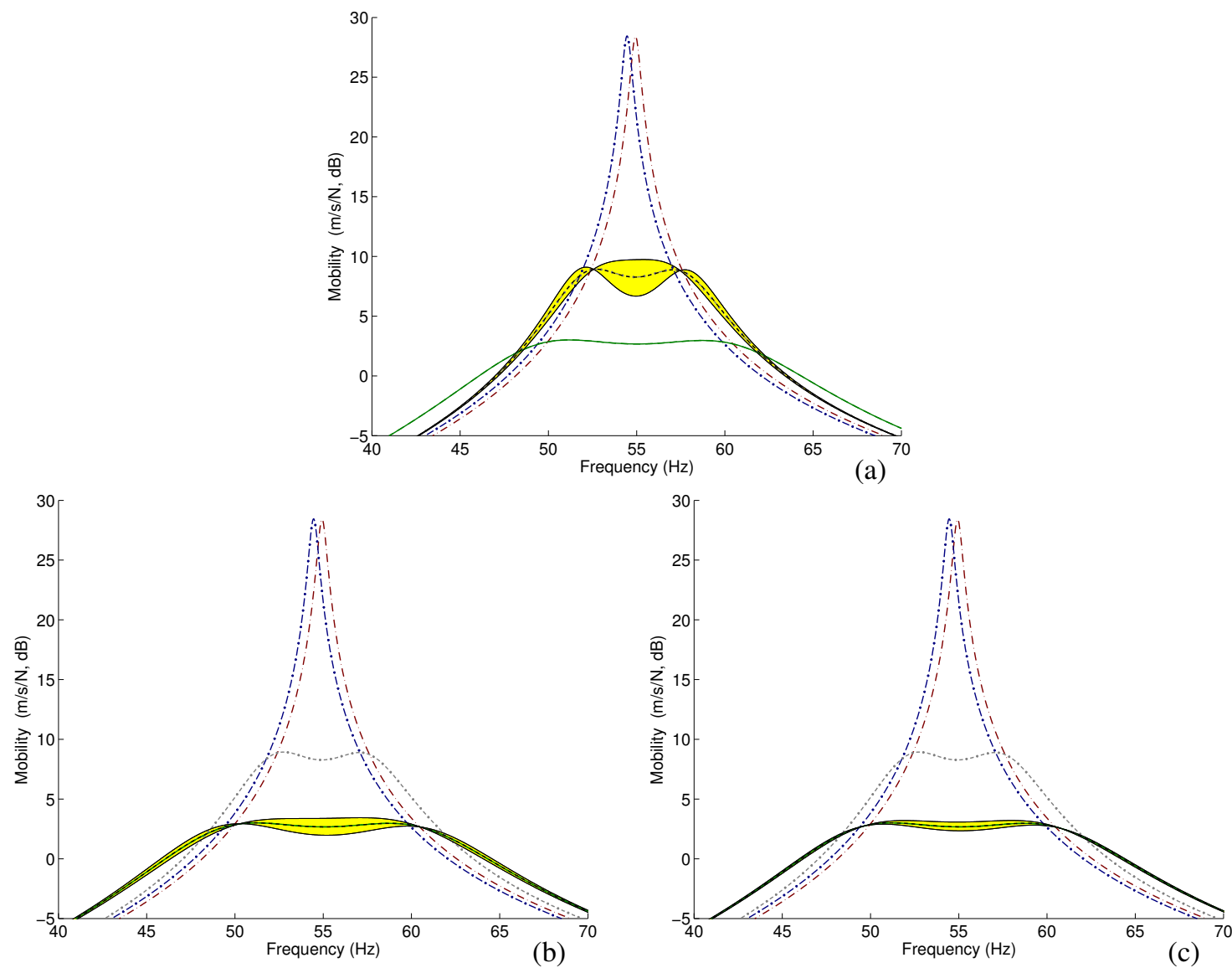

Figura 4.6: Análise estocástica para o primeiro modo com incerteza em $R_{c}$ no circuito elétrico (a) Passivo Puro, (b) Ativo-Passivo com ganho constante e (c) Ativo-Passivo com ganho variável.

que foram utilizadas as 5000 realizações variando somente a propriedade piezelétrica $L_{c}$ para gerar o intervalo de confiança. Analisando as respostas para as incertezas para o componente do circuito shunt $\left(L_{c}\right)$ o intervalo de confiança mostrou uma redução de 28 para $10 \mathrm{~dB}$ com o valor médio e de 28 para $16.4 \mathrm{~dB}$ para o limite superior.

Realizou-se uma análise da influência do controle neste caso, onde observou-se que, como apresentado pela figura 4.7(b) com ganho constante, a redução entre a estrutura em circuito aberto e o limite superior foi de $21.5 \mathrm{~dB}$. No caso apresentado pela figura 4.7(c) com ganho variável, a redução entre a estrutura em circuito aberto e o limite superior foi de $23.3 \mathrm{~dB}$. 

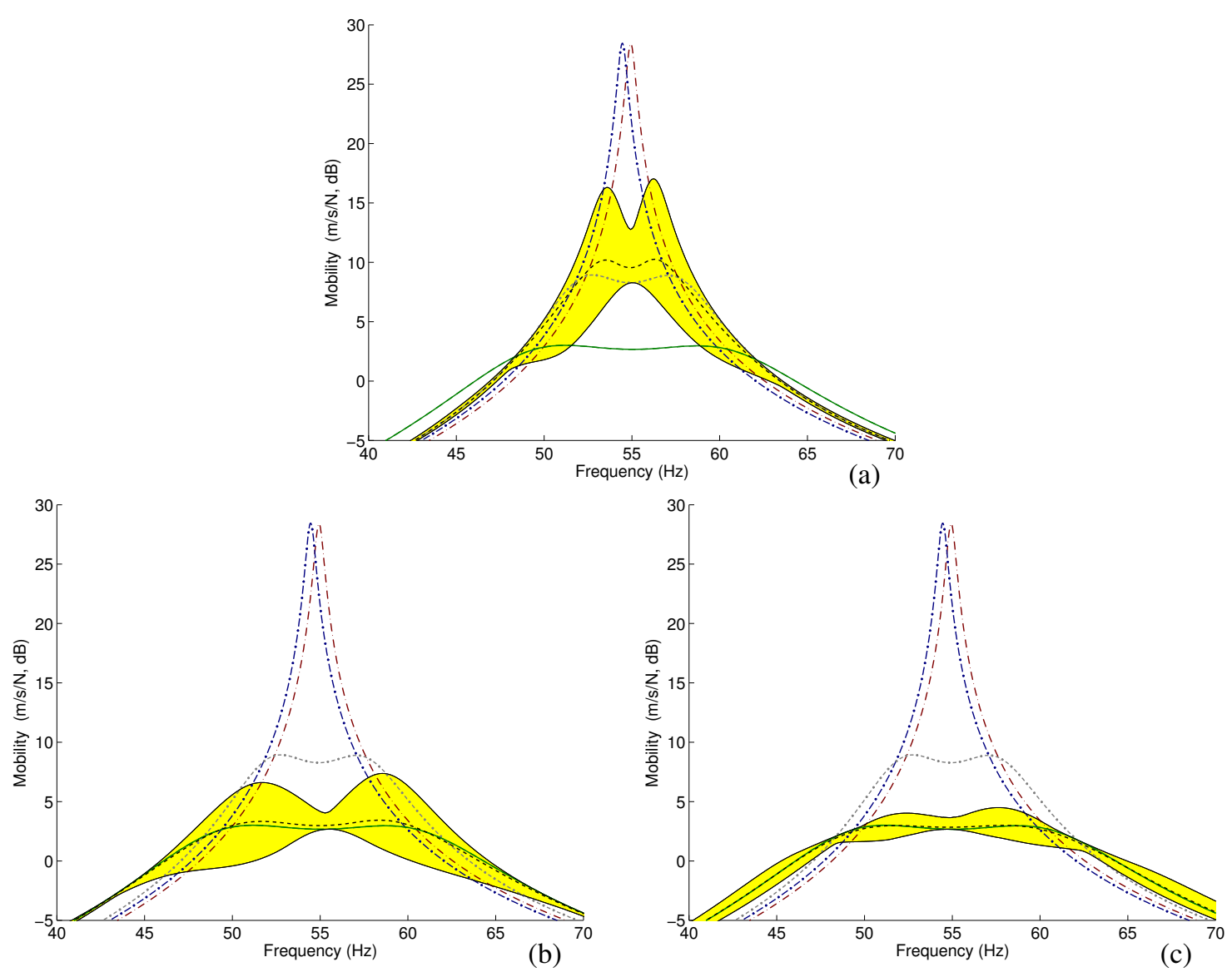

Figura 4.7: Análise estocástica para o primeiro modo com incerteza em $L_{c}$ no circuito elétrico (a) Passivo Puro, (b) Ativo-Passivo com ganho constante e (c) Ativo-Passivo com ganho variável.

\subsubsection{Análise da influência das incertezas para todas as variáveis con- sideradas}

Uma análise realizada considerando a configuração em extensão da estrutura proposta acima pode ser vista na figura 4.8, onde a resposta em frequência em circuito aberto (OC) $\left(G_{p}^{O C}\right)$ e conectada a um circuito shunt otimizado $\left(G_{p}^{N}\right)$ pode ser observada, lembrando que foram utilizadas as 5000 realizações variando todas as variáveis consideras e desta forma geramos o intervalo de confiança. Observamos que o circuito shunt RL não afetou a resposta em frequência nos modos adjacentes ao modo ajustado. Neste estudo, foi realizado um ajuste somente para o primeiro modo como pode se observar na Figura 4.8. 
Observa-se uma redução de $28 \mathrm{~dB}$, considerando a resposta do pico para a estrutura em circuito aberto, para $8 \mathrm{~dB}$ para a conectada no circuito shunt RL. Quando analisamos a resposta da estrutura para o caso onde temos o circuito shunt RL ativo com uma lei de controle, observamos uma redução para $3 \mathrm{~dB}$.

Analisando as respostas para as incertezas para todas as variáveis consideradas, o intervalo de confiança mostrou uma redução de 28 para $11 \mathrm{~dB}$ com o valor médio e de 28 para 20 dB para o limite superior. Realizou-se uma análise da influência do controle neste caso, onde observou-se que, como apresentado pela figura 4.8(b) com ganho constante, a redução entre a estrutura em circuito aberto e o limite superior foi de $18.3 \mathrm{~dB}$. No caso apresentado pela figura 4.8(c) com ganho variável, a redução entre a estrutura em circuito aberto e o limite superior foi de $21.5 \mathrm{~dB}$. 

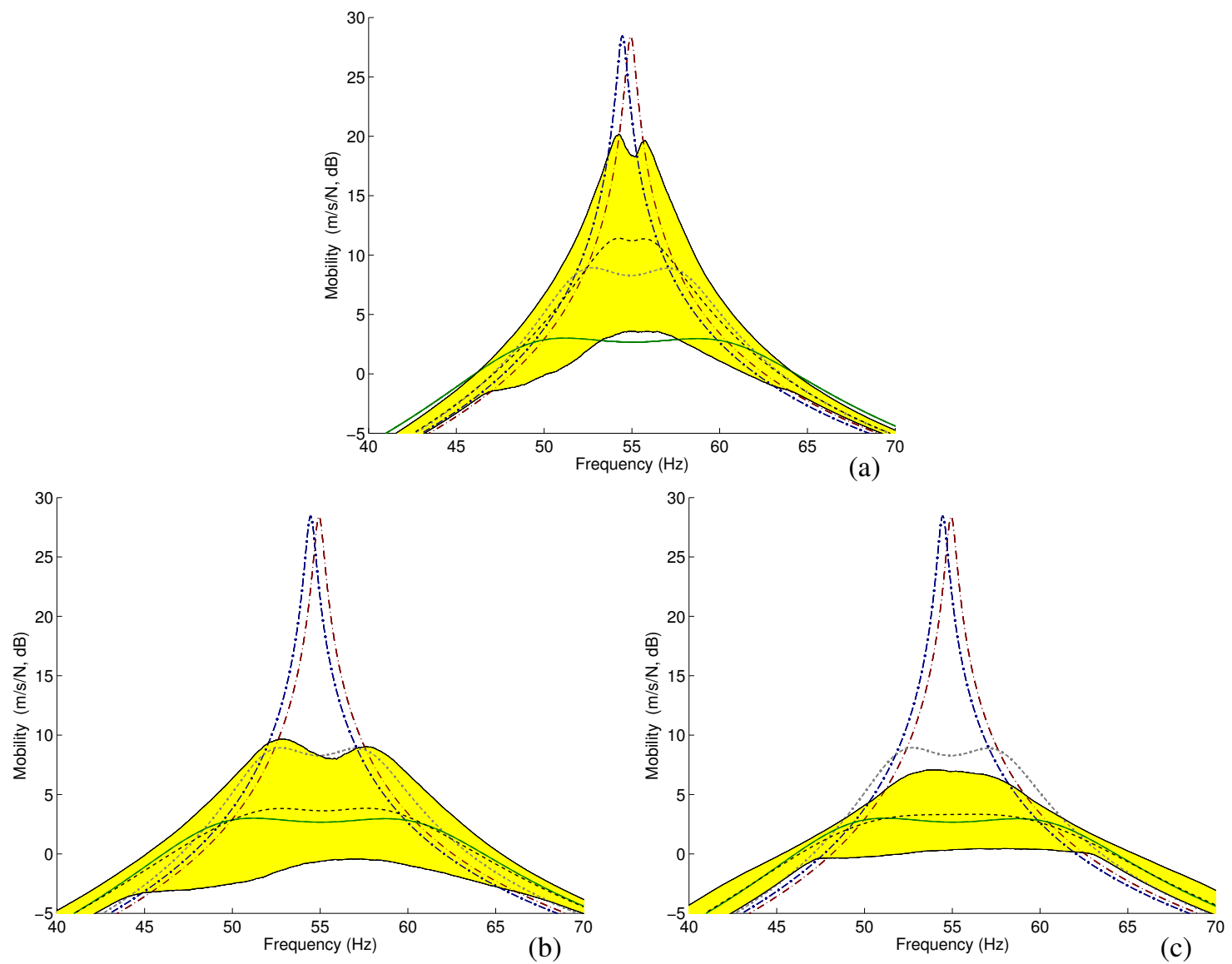

Figura 4.8: Análise estocástica para o primeiro modo com incerteza em todas as variáveis consideradas (a) Passivo Puro, (b) Ativo-Passivo com ganho constante e (c) Ativo-Passivo com ganho variável. 


\section{Capítulo 5}

\section{Otimização da resistência e indutância}

\section{em função da tensão aplicada}

Neste capítulo, apresenta-se resultados sobre a influência da tensão aplicada sobre os componentes do circuito elétrico que compõe o APPN. Será realizada uma análise sobre a autoridade de controle em estruturas utilizando o controle ativo-passivo (APPN), sendo que os valores dos componentes do circuito elétrico são adequados para cada valor de tensão aplicada. Estes valores serão adequados para o primeiro modo de vibração de uma estrutura do tipo placa e uma análise das respostas em frequência é realizada demonstrando a sua eficiência. A otimização dos componentes do circuito elétrico, definidos como sendo resistência, indutância e tensão aplicada, é realizada através de um algoritmo genético.

\subsection{Definição do problema a ser otimizado}

Nesta seção é proposta uma nova estrutura, se diferenciando das anteriores até então apresentadas. Esta nova estrutura é uma placa, conforme a Figura 5.2, sendo engastada em todos os seus lados com dimensões $500 \mathrm{~mm}$ de comprimento, $400 \mathrm{~mm}$ de largura e com $1 \mathrm{~mm}$ de espessura. Os elementos piezelétricos acoplados em sua superfície através de 
uma camada de cola de $0.1 \mathrm{~mm}$ de espessura em toda área em contato entre as pastilhas piezelétricas e a placa, localizado a $5 \mathrm{~mm}$ dos lados engastados.

Nesta configuração, considera-se cerâmicas piezelétricas são feitas de PZT-5H e foram as propriedades consideradas do material piezelétrico: $\bar{c}_{11}^{D}=\bar{c}_{22}^{D}=97.767 \mathrm{GPa}$, $\bar{c}_{12}^{D}=50.774 \mathrm{GPa}, \bar{c}_{44}^{D}=\bar{c}_{55}^{D}=42.217 \mathrm{GPa}, \rho=7500 \mathrm{~kg} \mathrm{~m}^{-3}$, constante de acoplamento piezelétrica $\bar{h}_{31}=-1.352010^{9} \mathrm{~N} \mathrm{C}^{-1}$ e e constante dielétrica $\bar{\beta}_{33}^{\varepsilon}=57.83010^{6} \mathrm{~m} \mathrm{~F}^{-1}$. Para a placa de alumínio, as propriedades do material são definidas por: Módulo de Young $70.3 \mathrm{GPa}$ e densidade $2710 \mathrm{~kg} \mathrm{~m}^{-3}$, um amortecimento viscoso de $0.5 \%$ foi considerado. A cola utilizada tem um Módulo de Young de $2.5 \mathrm{GPa}$ e densidade $1126 \mathrm{~kg} \mathrm{~m}^{-3}$.

Várias estruturas foram estudadas e diversas posições dos elementos piezelétricos foram considerados, sendo que alguns exemplos são mostrados na figura 5.1. Em todos os casos uma excitação foi aplicada no centro das placas e a leitura de saída realizada no mesmo ponto.

Após algumas análises preliminares a geometria apresentada na figura 5.2 foi escolhida. Esta escolha visa o favorecimento da deformação das pastilhas piezelétricas e a ausência de pastilhas no centro da placa maximizando a área livre.

Para esta estrutura, uma otimização nos valores de resistência e indutância em função da tensão aplicada no circuito elétrico, será realizada com o objetivo de garantir a máxima redução na amplitude de vibração da estrutura tipo placa definida para o primeiro modo de vibração. Para este processo de otimização utilizaremos um algoritmo genético que busca uma minimização de uma função custo definida como sendo a máxima redução da amplitude de vibração para o primeiro modo da estrutura.

Uma análise foi realizada considerando a configuração descrita na Figura 5.2 onde analisou-se a resposta em frequência com circuito elétrico composto de uma resistência, uma indutância e uma fonte de tensão aplicando-se uma lei de controle LQR, buscando minimizar o máximo valor da resposta em frequência em um intervalo pré definido nas proximidades da primeira ressonância. 

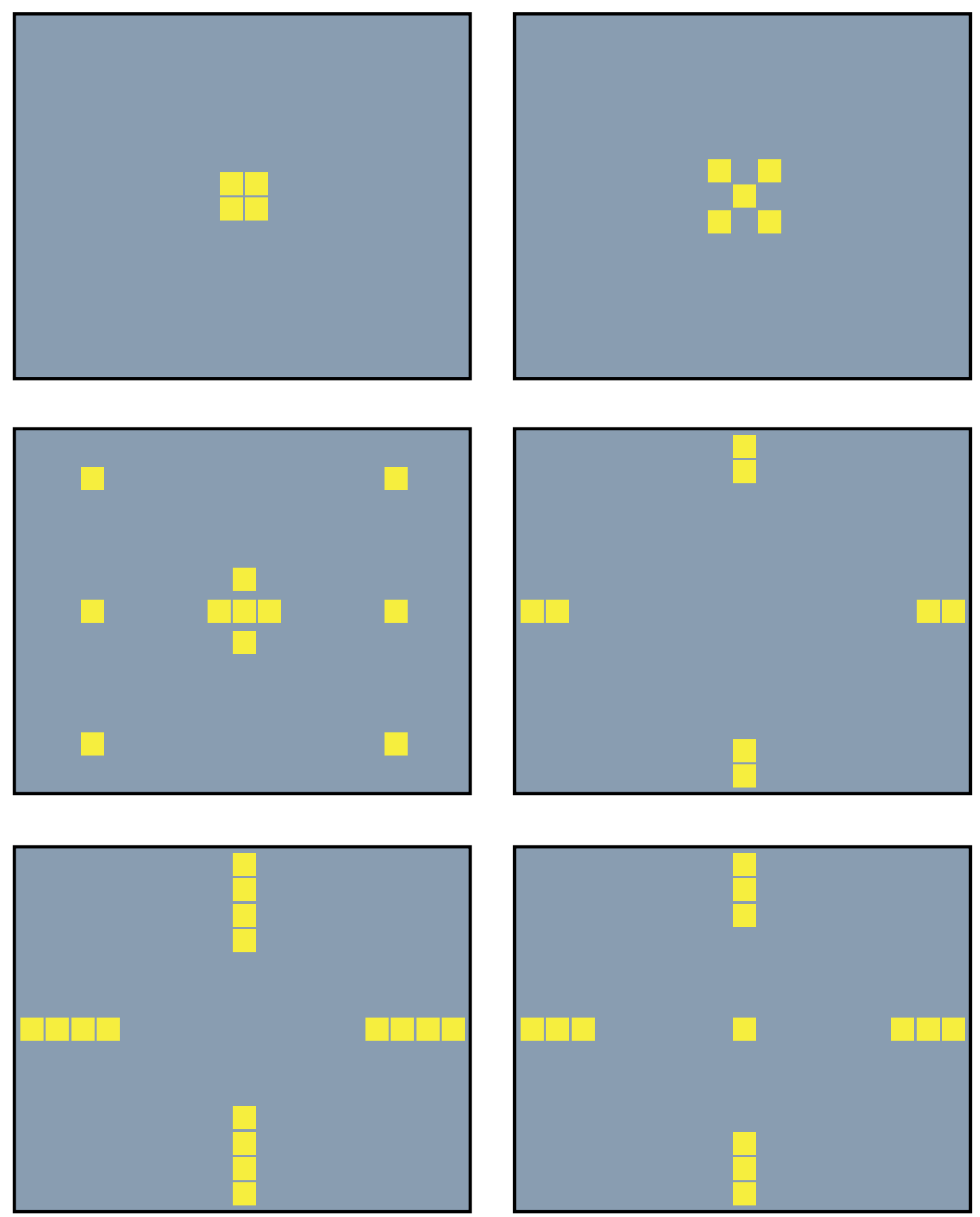

Figura 5.1: Esquema de estruturas do tipo placa utilizadas para busca de uma nova estrutura para o processo de otimização com circuito elétrico conectado nas pastilhas piezelétricas.

\subsection{Metodologia de otimização dos parâmetros de cir- cuito elétrico utilizados para o APPN}

Para que o processo de otimização seja realizado, é necessário definir uma função objetivo para o problema a ser estudado. Para isso, definimos o objetivo do estudo como sendo, 

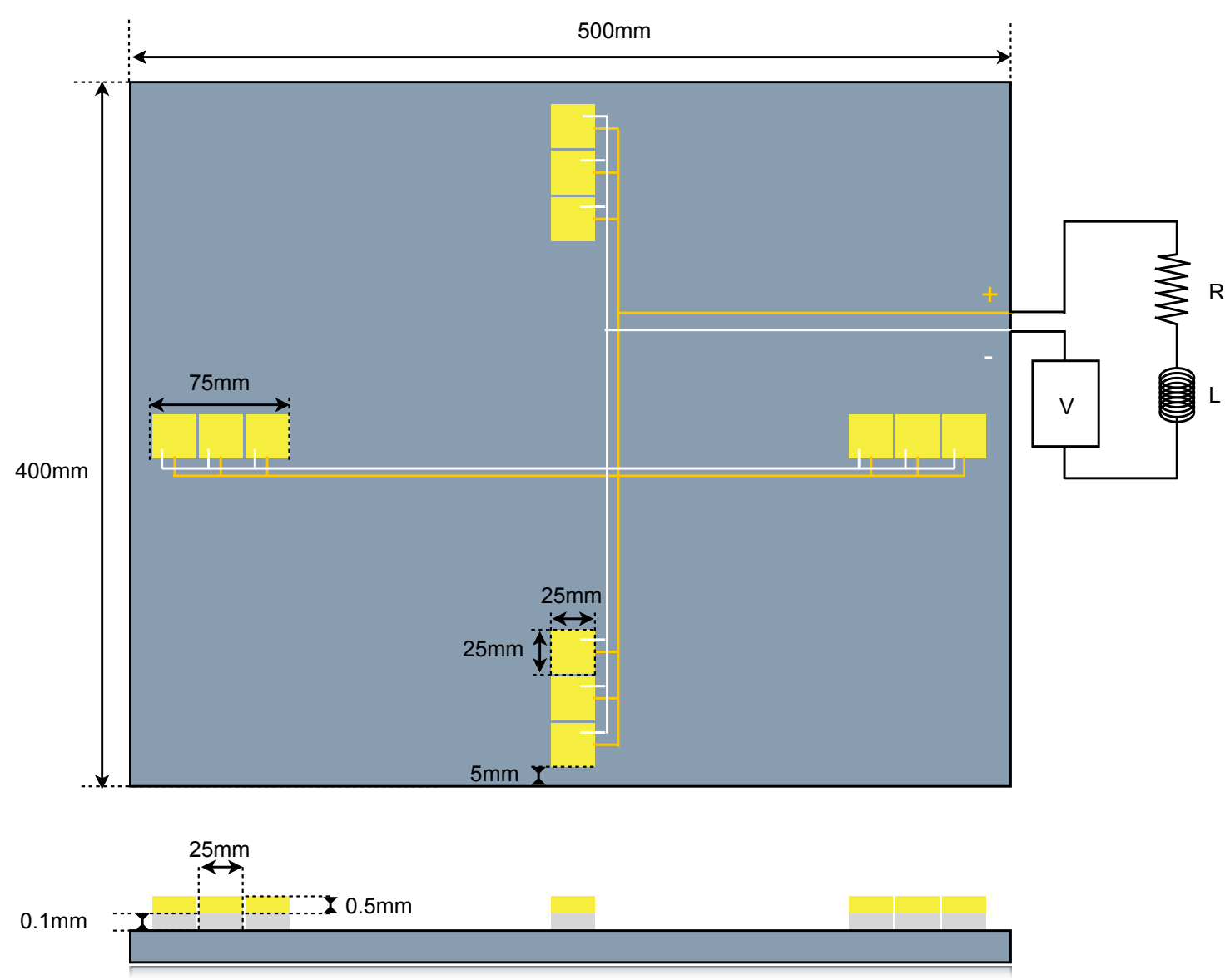

Figura 5.2: Esquema da estrutura tipo placa escolhida utilizada para otimização com circuito elétrico conectado nas pastilhas piezelétricas.

identificar a máxima amplitude da resposta em frequência, dada pela equação (3.56) em um dado intervalo de frequência, $[40-90] \mathrm{Hz}$, tal que

$$
J_{o p}=\max \left[\left|G_{h}(\omega)\right|\right]
$$

para $40<\frac{\omega}{2 \pi}<90$, seja minimizado.

A metodologia considerada, consiste em buscar os valores de resistência e indutância para circuito elétrico que minimize $J_{o p}$ para vários valores de tensão máxima aplicada pelo controle ativo. Um limite inferior e superior foi imposto para cada uma das variáveis de forma a limitar o espaço de soluções que foi construído, agilizando assim a otimização. Os limites impostos para o valor da resistência foram $0 \leq R \leq 10 R_{o p}$ e para a 
indutância os limites impostos foram $0 \leq L \leq 2 L_{o p}$, sendo $R_{o p}$ e $L_{o p}$ são os valores ótimos para o caso passivo ótimo (tensão de controle nula).

O processo de otimização foi realizado utilizando uma ferramenta de algoritmo genético encontrada no software comercial MATLAB ${ }^{\circledR}$. Esta ferramenta possui diversas funções para operações de reprodução, mutação e seleção. Neste trabalho foram consideradas as seguintes funções: para a reprodução, uma reprodução dispersiva, para mutação, uma mutação gaussiana e para a seleção, uma seleção uniforme estocástica na qual a probabilidade de um indivíduo ser escolhido depende da função objetivo correspondente. A operação de reprodução foi considerada para $40 \%$ da população, uma elite dos 5 melhores indivíduos de cada geração foi mantida, sendo a operação de mutação considerada para o restante da população. Uma população inicial de 1000 indivíduos foi construída aleatoriamente, sendo cada individuo composto por valores de resistência $(R)$ e indutância $(L)$ respeitando os limites impostos anteriormente. Foi considerado um número máximo de 60 gerações para evolução sendo que nos casos estudados apenas 51 gerações foram suficientes para convergência da função objetivo.

Definida a população inicial a metodologia de otimização descrita anteriormente foi realizada para diferentes valores de tensão máxima aplicada entre $10 \mathrm{~V}$ até $250 \mathrm{~V}$ variando de 10 em $10 \mathrm{~V}$. Além disso três diferentes amplitudes da força de perturbação foram consideradas, sendo $F_{1}, F_{2}$ e $F_{3}$ as forças de perturbação impulsivas que geram os deslocamentos máximos $h, h / 2$ e $h / 5$, respectivamente, para o centro da placa, onde $h=1 \mathrm{~mm}$ é a espessura da placa considerada.

\subsection{Resultados da otimização para estrutura tipo placa com L de $500 \mathrm{~mm}$}

A figura 5.3 apresenta a variação dos valores de resistência e indutância em função da tensão aplicada para $F_{1}, F_{2}$ e $F_{3}$, pode-se observar que os valores de indutância não variam 
84 Capítulo 5. Otimização da resistência e indutância em função da tensão aplicada

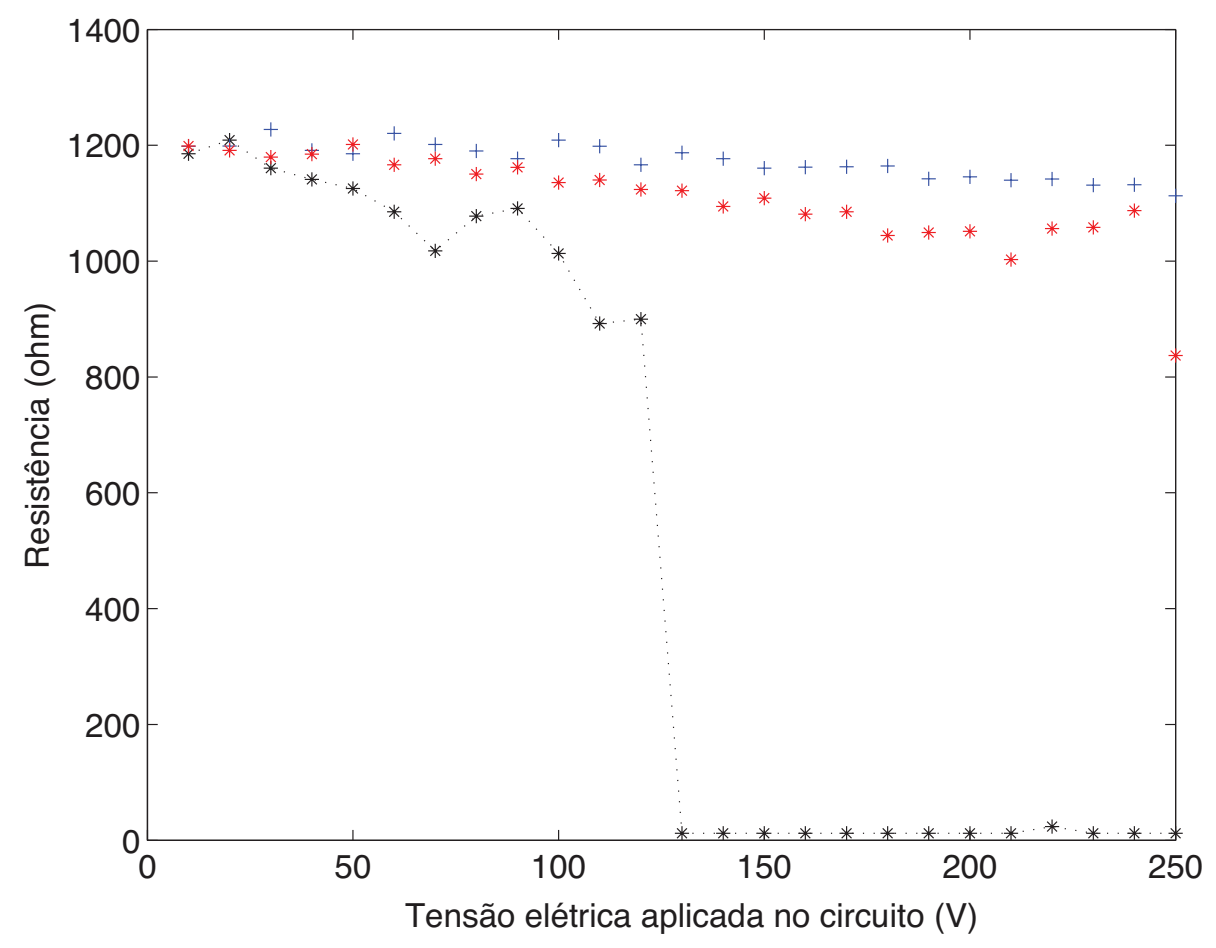

(a)

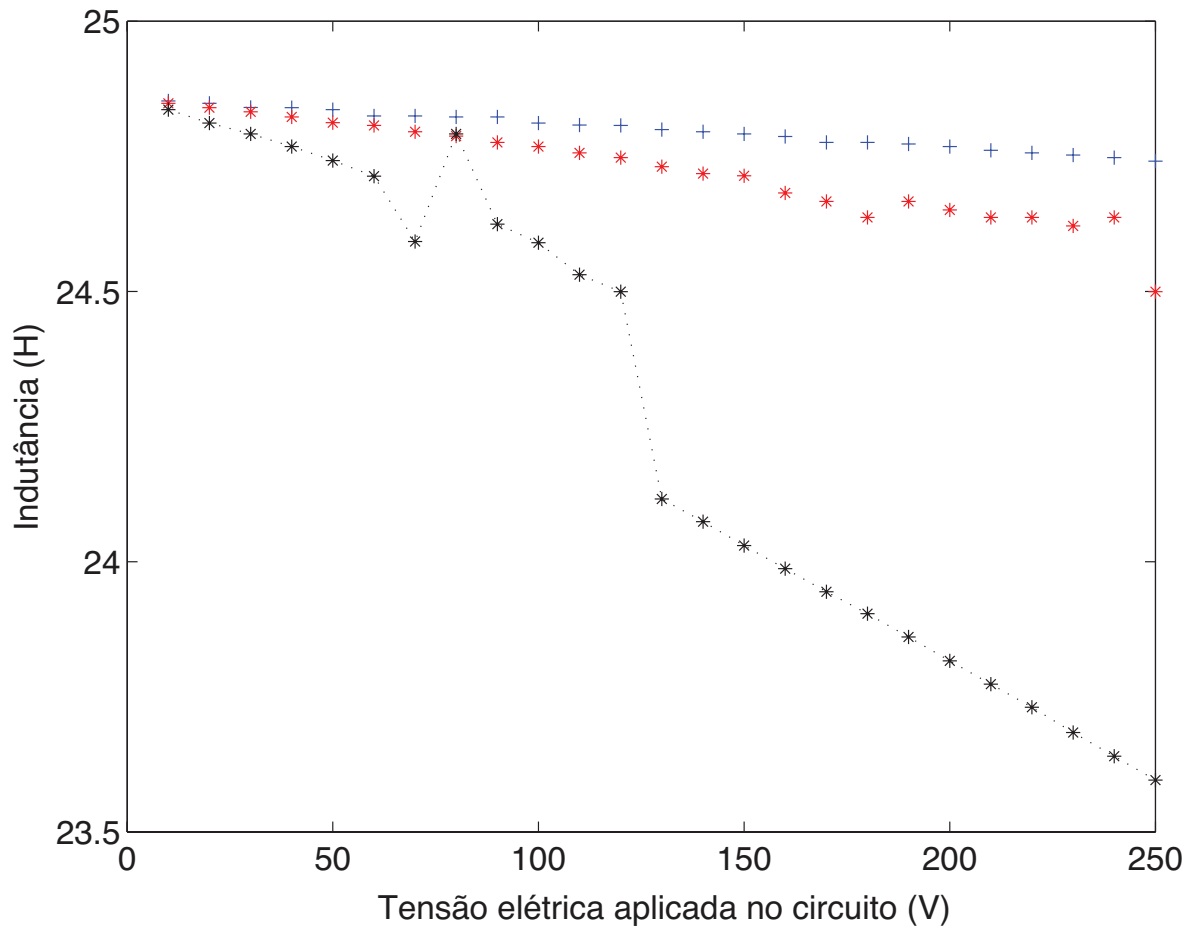

(b)

Figura 5.3: Variação da (a) resistência e (b) indutância em função da tensão aplicada para placa de $500 \mathrm{~mm}$ x $400 \mathrm{~mm}$ com $F_{1}(+$ azul $) ; F_{2}\left(*\right.$ vermelho) e $F_{3}(*$ - preto). 
muito para as tensões aplicadas. Desta forma uma análise mais detalhada pode ser realizada para a figura 5.3(a) com amplitude da força de perturbação $F_{1}$, definida pela curva azul. A variação dos valores de resistência $R$ é muito pequena indo de $R=1.2273 \mathrm{k} \Omega$ para o máximo valor obtido até $R=1.1129 \mathrm{k} \Omega$ para o menor valor obtido. Uma análise na figura 5.3 mostra que o decaimento no máximo valor da resposta em frequência está no intervalo de $[9.3-10.6] \mathrm{dB}$ de maneira contínua.

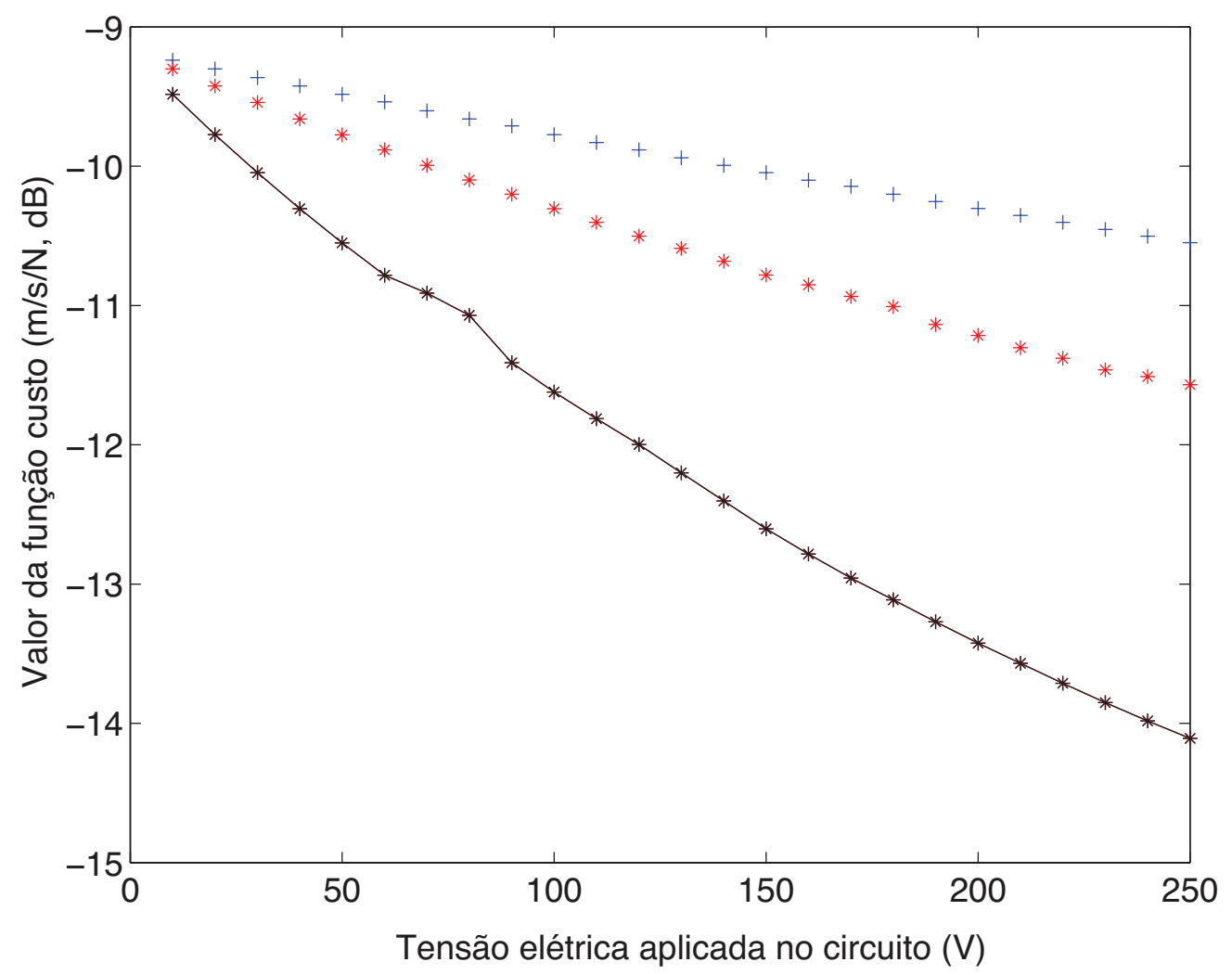

Figura 5.4: Variação da amplitude da resposta em frequência em função da tensão aplicada para placa de $500 \mathrm{~mm}$ x $400 \mathrm{~mm}$ com diferentes $F_{1}(+$ azul $) ; F_{2}\left(*\right.$ vermelho) e $F_{3}\left(*_{-}\right.$ preto).

Quando analisamos a mesma figura, agora para a amplitude de força de perturbação $F_{2}$, temos uma variação maior dos valores de resistência, sendo $R=1.2015 \mathrm{k} \Omega$ para o máximo valor obtido e $R=837.1800 \Omega$ para o menor valor obtido. De maneira análoga ao caso anterior, ao observar a figura 5.3 o decaimento do máximo valor da resposta em frequência fica compreendido no intervalo de $[9.3-11.2] \mathrm{dB}$, também ocorre de maneira 
contínua.

Uma análise mais criteriosa será feita para o caso com amplitude de força de perturbação $F_{3}$. Neste caso temos uma variação maior dos valores de resistência, sendo $R=1.2090 \mathrm{k} \Omega$ e valor minimo de $R=12.0554 \Omega$, ao se analisar a Figura 5.3 observase um decaimento abrupto dos valores de resistência em $V_{\max }=120 \mathrm{~V}$ e $V_{\max }=130 \mathrm{~V}$ gerando uma descontinuidade que será estudada na próxima seção.

A Figura 5.4 mostra que para todos os casos estudados, $\left(F_{1}, F_{2}\right.$ e $\left.F_{3}\right)$, o valor ótimo para a função objetivo decresce com a tensão aplicada no circuito elétrico. Isto é um indicativo que, independente dos valores ótimos de resistência e indutância otimizados, o aumento da tensão máxima sempre dá origem a uma redução da amplitude de vibração. Também pode-se observar que para valores menores de perturbação, como no caso $\left(F_{3}\right)$, a redução dos níveis de amplitude é mais pronunciada e pode chegar até $14 \mathrm{~dB}$. 


\subsection{Resposta em frequência para estruturas com $R$ e $L$ otimizados}

Nesta seção iremos analisar o comportamento da estrutura tipo placa proposta, através da sua resposta em frequência, para diversos valores otimizados de resistência e indutância. Estes valores foram obtidos através do método de algoritmos genéticos, já descrito na seção inicial deste capítulo.

Para isto realizaremos uma análise das respostas em frequências em função dos valores de tensão elétrica aplicada considerados na otimização. Esta análise é necessária para o entendimento da descontinuidade apresentada na Figura 5.3, presente ao se analisar o comportamento dos valores de resistência e indutância para uma amplitude de força igual $F_{3}$. Uma maneira de se visualizar o que acontece com a estrutura é observar a resposta em frequência.

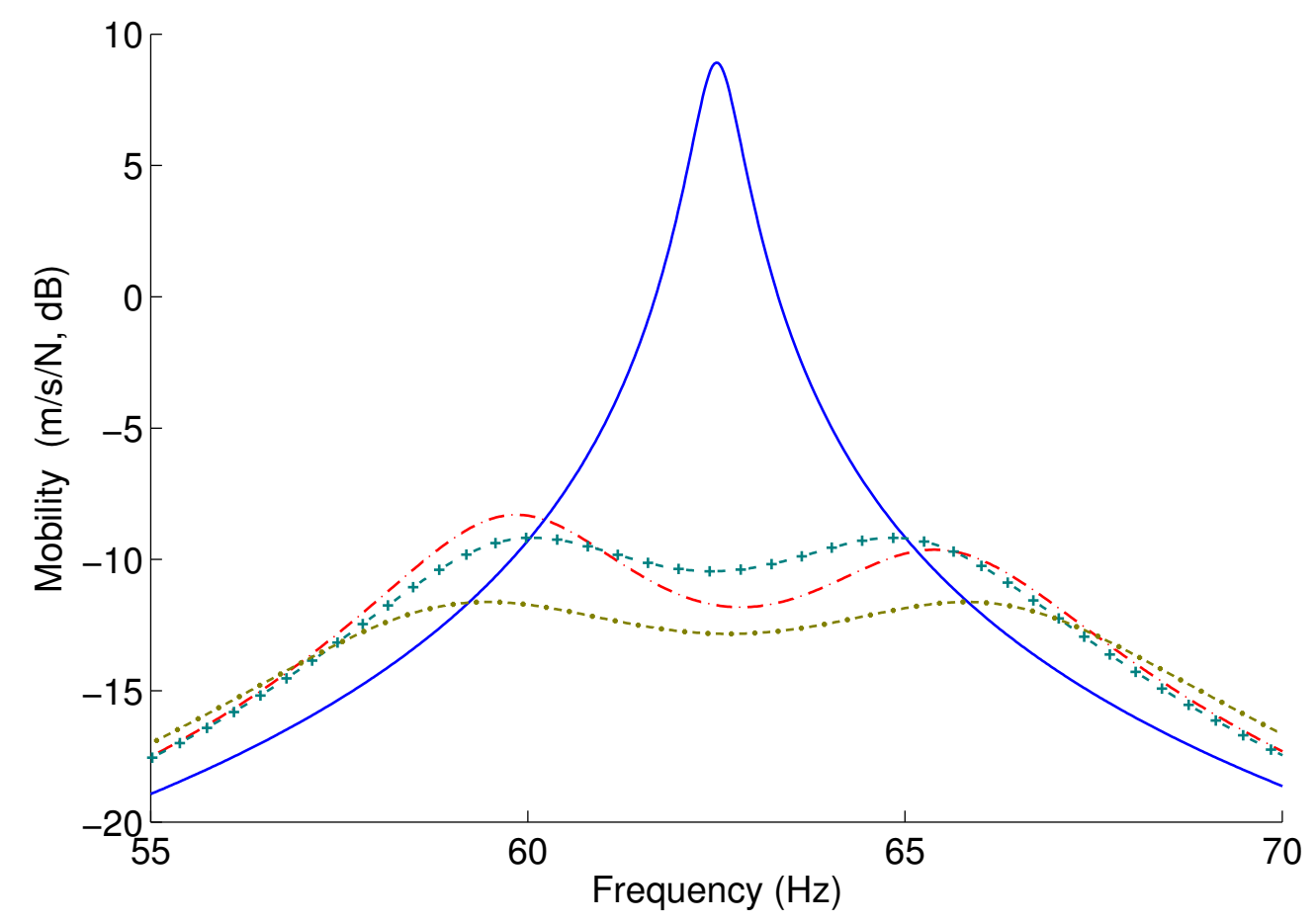

Figura 5.5: Resposta da estrutura nos casos: circuito aberto (azul), passivo ótimo (verde), passivo (vermelho) e ativo-passivo (amarelo) com tensão de $V_{\max }=100 \mathrm{~V}$. 


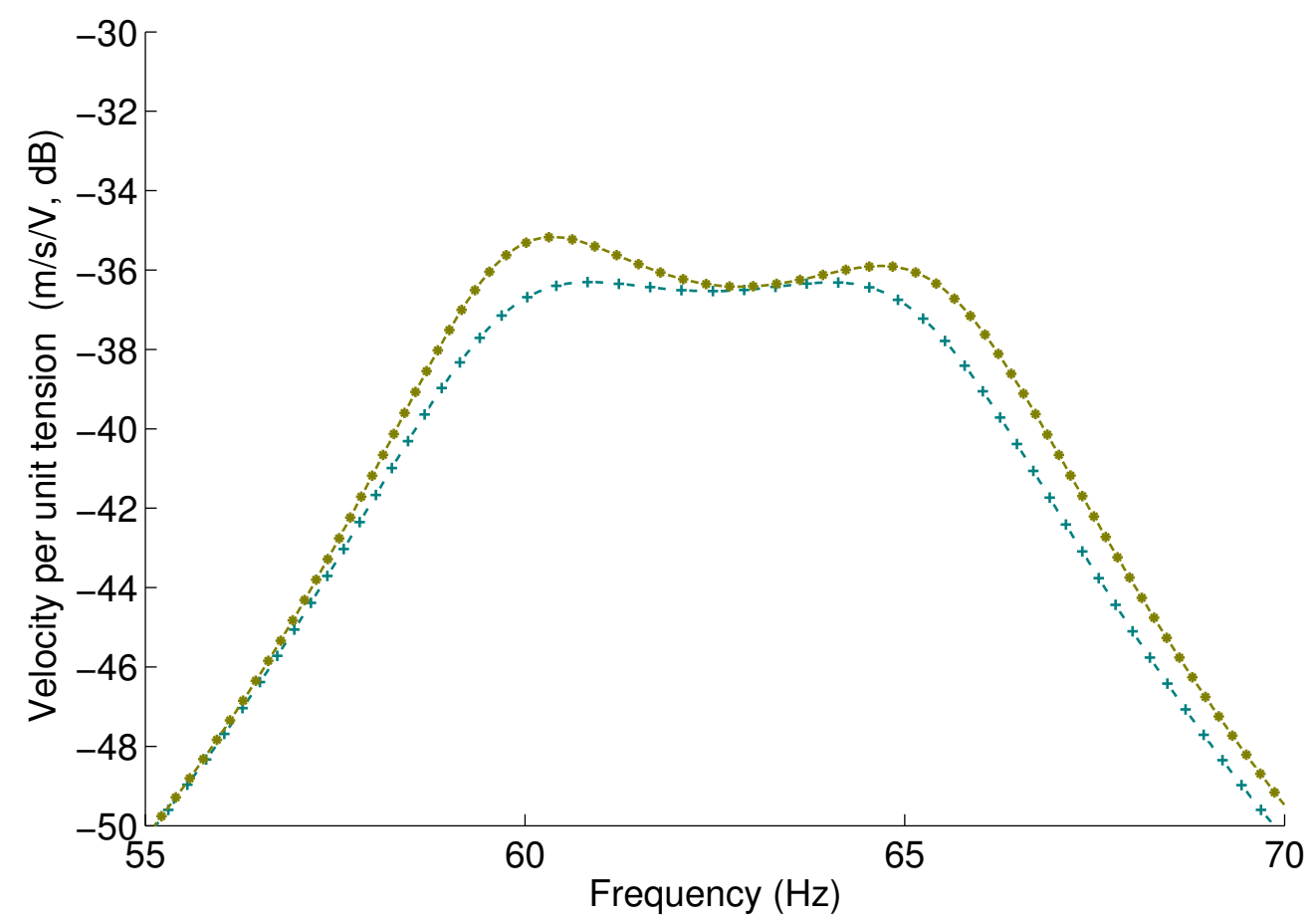

Figura 5.6: Autoridade de controle para valores de resistência e indutância otimizados para os casos puramente V (verde) e RLV com $V_{\text {max }}=100 \mathrm{~V}$ (amarelo).

Na Figura 5.5 podemos analisar a resposta em frequência do primeiro modo de vibração da estrutura com circuito elétrico conectado, utilizando os valores otimizados de resistência e indutância para caso passivo ótimo traço - traço verde. Através da otimização por algoritmo genético, o valor obtido para a resistência foi de $R=1.2055 \mathrm{k} \Omega$ e para a indutância foi de $L=24.8557 H$. Realizando uma comparação com a resposta em frequência da estrutura em circuito aberto, curva $a z u l$, observamos uma redução na amplitude de vibração de $17.5 \mathrm{~dB}$. Calculando a resposta em frequência para a estrutura em modo passivo, $\left(V_{\max }=0\right)$, com os valores de resistência e indutância otimizados para $V_{\max }=100 \mathrm{~V}, R=1.0133 \mathrm{k} \Omega$ e $L=24.8114 \mathrm{H}$, podemos observar que temos uma redução de $17 \mathrm{~dB}$ (curva -. - vermelho). O desempenho do sistema passivo neste caso, é menor quando comparado com o caso passivo ótimo, porém ao se adicionar o controle ativo o desempenho final se torna melhor com redução de $22 \mathrm{~dB}$ (curva -. - amarelo).

Este fenômeno pode ser explicado pelo fato que, ao mesmo tempo que o desempe- 


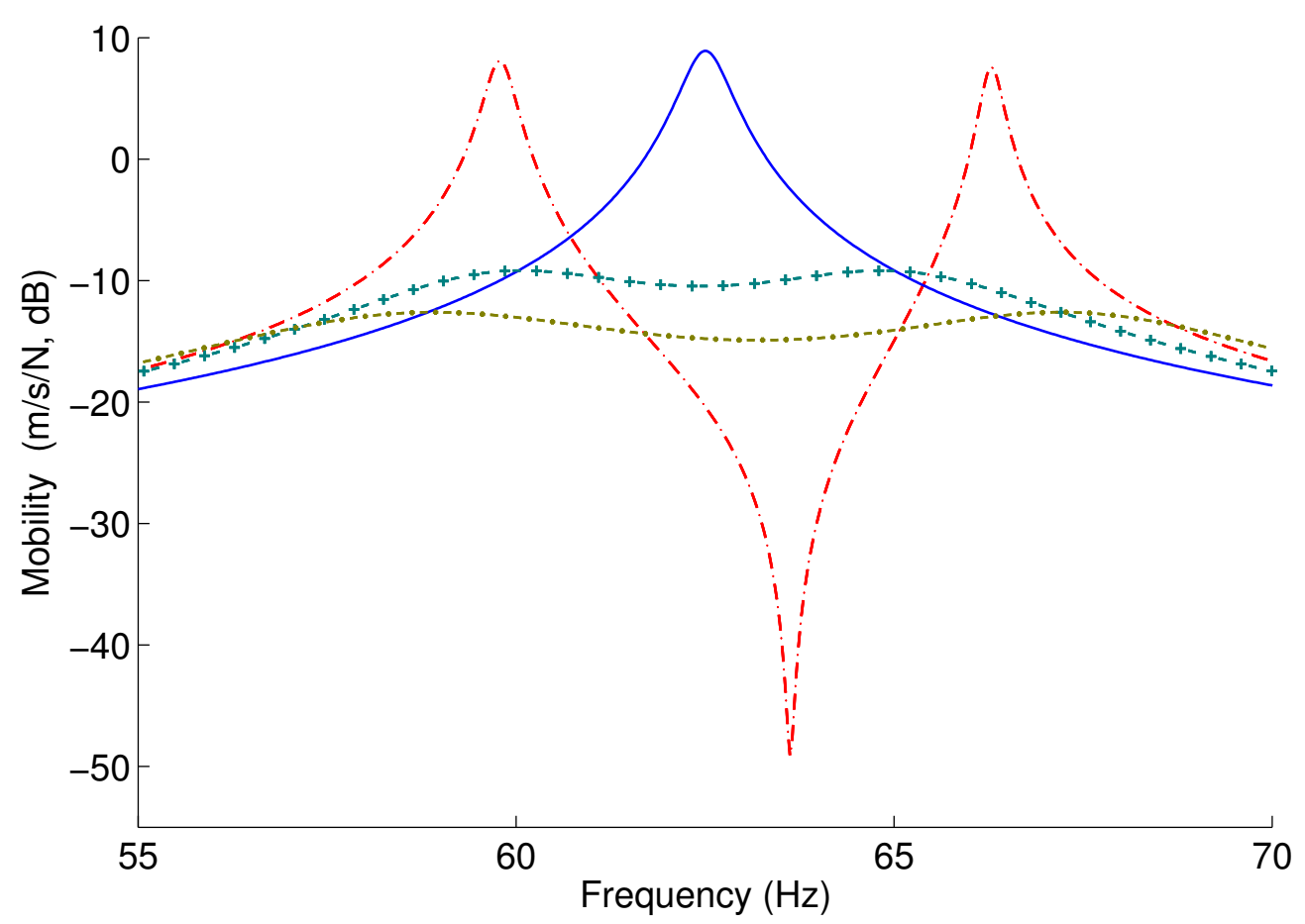

Figura 5.7: Resposta da estrutura nos casos: circuito aberto (azul), passivo ótimo (verde), passivo (vermelho) e ativo-passivo (amarelo) com tensão de $V_{\max }=150 \mathrm{~V}$

nho do controle passivo é piorado pela modificação dos valores de resistência e indutância otimizados, a autoridade de controle das pastilhas piezelétricas é aumentada. Este efeito pode ser observado na Figura 5.6 que apresenta o gráfico de autoridade de controle para valores de resistência e indutância otimizados os casos V e RLV (utilizando a equação 2.3) para o caso puramente passivo e ativo-passivo. Pode-se observar que a autoridade de controle é aumentada na região da frequência de ressonância para o caso ativo-passivo (curva -. - amarelo).

Na Figura 5.7 podemos também analisar a resposta em frequência do primeiro modo de vibração da estrutura com circuito elétrico conectado, utilizando os valores otimizados de resistência e indutância para caso passivo ótimo traço-traço verde. Porém ao calcular a resposta em frequência para a estrutura em modo passivo, $\left(V_{\max }=0\right)$, com os valores de resistência e indutância otimizados para $V_{\max }=150 \mathrm{~V}, R=12.0555 \Omega$ e $L=24.0297 \mathrm{H}$, podemos observar que não observamos uma redução amplitude, (curva - . - vermelho), 


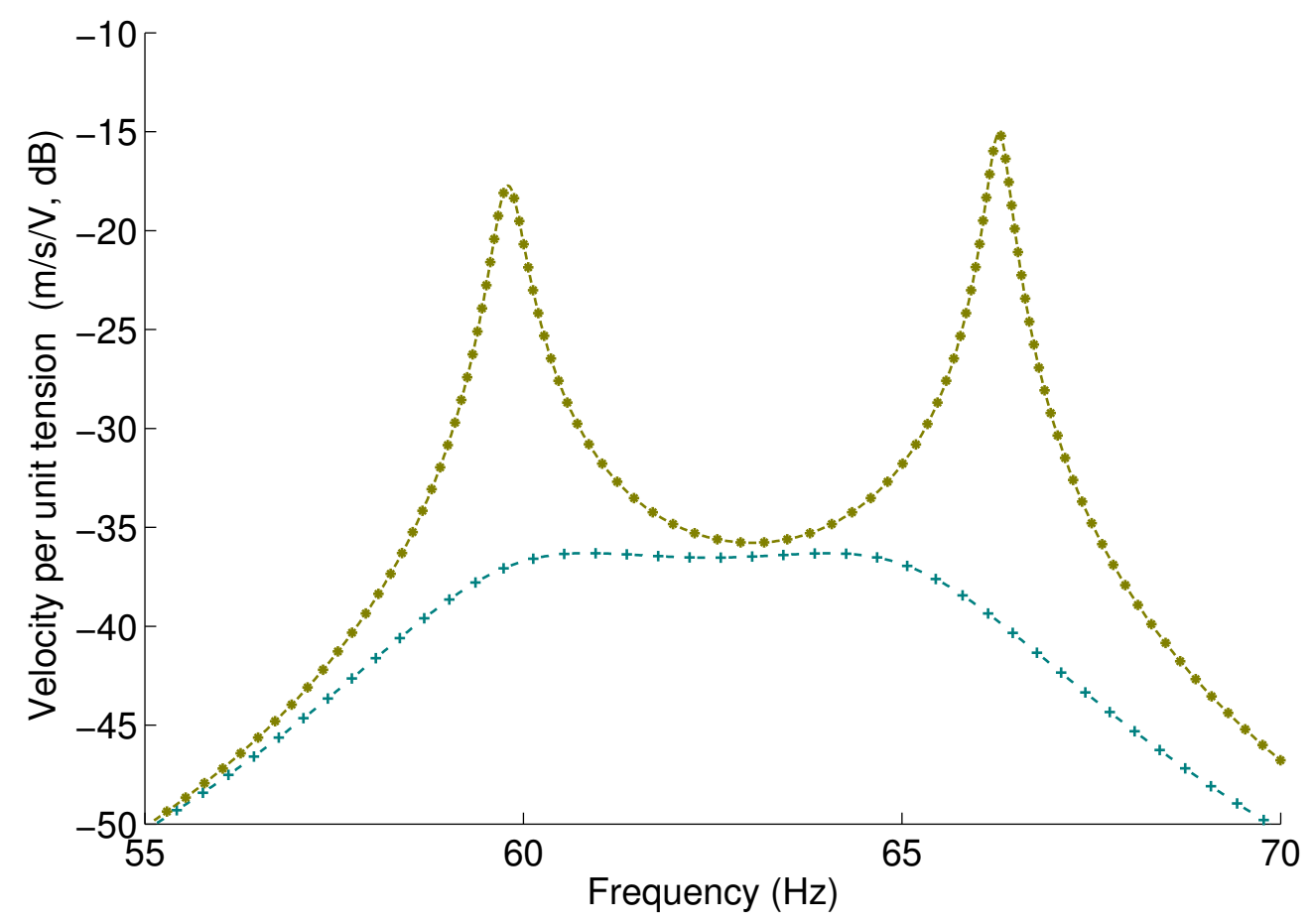

Figura 5.8: Autoridade de controle para valores de resistência e indutância otimizados para os casos puramente passivo (verde) e ativo-passivo com $V_{\max }=150 \mathrm{~V}$ (amarelo).

quando comparado ao caso em circuito aberto. O desempenho do sistema passivo neste caso, não só é menor quando comparado com o caso passivo ótimo, como apresenta um desajuste no controle proposto. No entanto, ao se adicionar o controle ativo o desempenho final se torna significativamente melhor com redução de $23 \mathrm{~dB}$ (curva -. - amarelo).

Neste caso, para $V_{\max }=150 \mathrm{~V}$, o fenômeno pode ser explicado pelo fato que, ao mesmo tempo que o desempenho do controle passivo é piorado pela modificação dos valores de resistência e indutância otimizados, a autoridade de controle das pastilhas piezelétricas é aumentada. Este efeito pode ser observado na Figura 5.8 e demonstra que valores baixos de resistência provocam picos assentuados na autoridade de controle. Neste caso, a utilização de valores de resistência baixas inviabiliza a utilização do controle em modo passivo. Porém, o desempenho do controle no modo ativo-passivo é muito superior ao caso passivo ótimo. Pode-se observar que a autoridade de controle é aumentada na região da frequência para o caso ativo-passivo (curva $* *$ amarelo). 
Este fenômeno explica a descontinuidade apresentada na seção anterior para os valores de resistência e indutância para $F_{3}$, pois ocorre uma mudança abrupta na autoridade de controle, onde para tensões baixas o melhor controle é puramente passivo, de maneira que quando a tensão elétrica aplicada sobre o circuito aumenta, a otimização dá preferência a parte ativa do controle passando a ser majoritariamente ativo.

Uma outra verificação pode ser feita traçando a resposta da estrutura para todos os valores otimizados de resistência e indutância juntamente com os valores de tensão. Na Figura 5.9 pode-se observar o comportamento monotônico de redução dos valores de amplitude, para $F_{1}(\mathrm{a}), F_{2}(\mathrm{~b})$ e $F_{3}(\mathrm{c})$.

Ao analisar os intervalos gerados pelas diferentes respostas em frequências, observa-se que a redução é maior para menores valores de amplitude de forças de perturbação. Uma outra análise que pode ser realizada é na Figura 5.9(c) onde podese observar a transição de um controle majoritariamente passivo para um controle majoritariamente ativo. Em todos os casos, o aumento da tensão elétrica sempre diminui a amplitude de vibração para o intervalo de frequência considerado. 

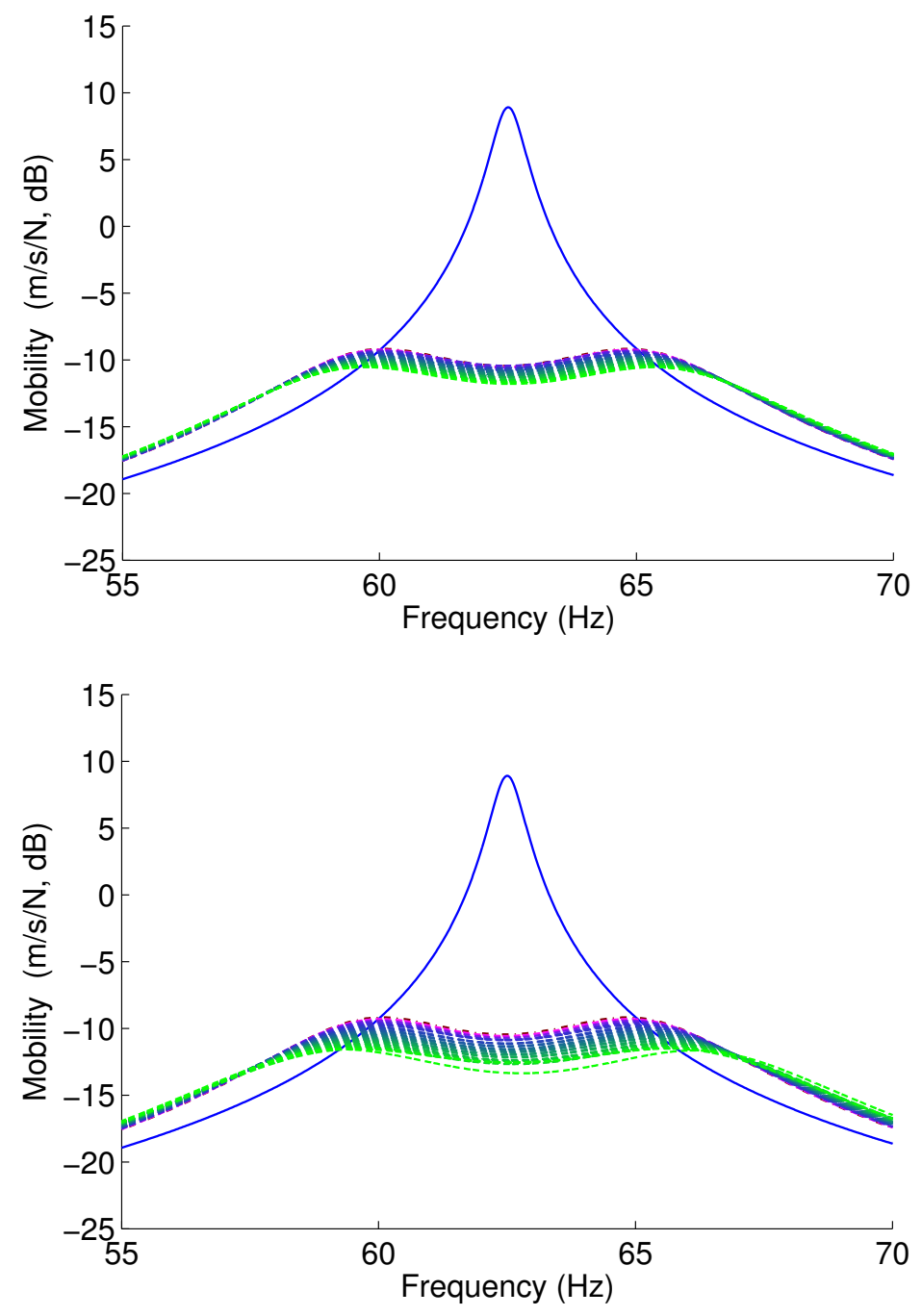

(a)

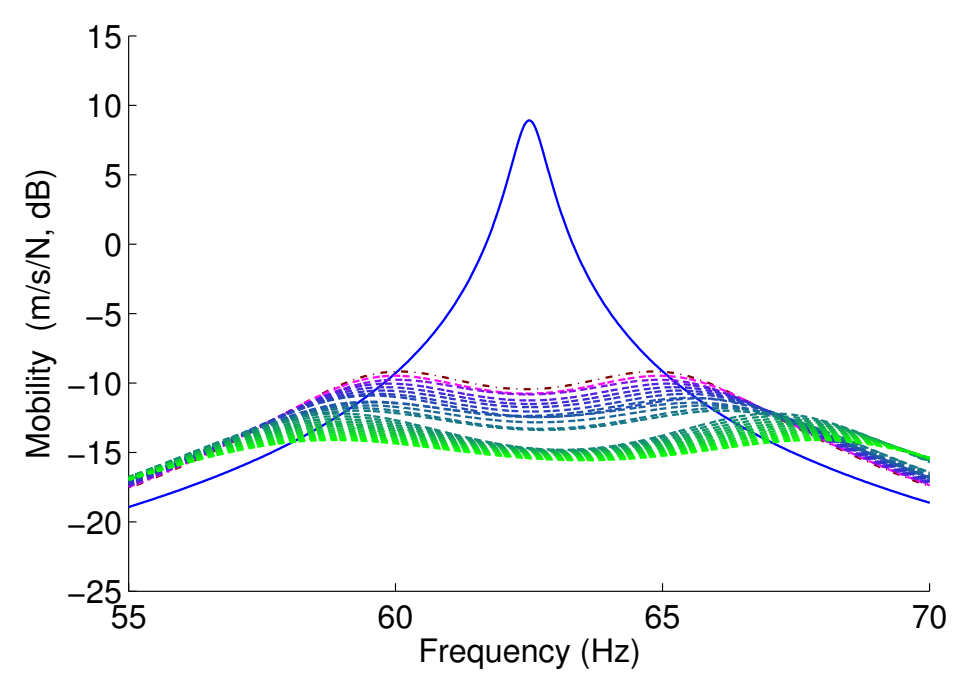

(b)

(c)

Figura 5.9: Resposta em frequência da estrutura em circuito aberto (continua azul) e com controle ativo-passivo (- -) para diferentes valores de tensão máxima no intervalo de 10 a $250 \mathrm{~V}$ e para diferentes amplitudes de força de perturbação $F_{1}(\mathrm{a}), F_{2}(\mathrm{~b})$ e $F_{3}(\mathrm{c})$ 


\section{Capítulo 6}

\section{Análise estocástica para incertezas na interface pastilha/placa (cola)}

Neste capítulo, apresenta-se um estudo da influência da incerteza do módulo de Young da cola que realiza o acoplamento das pastilhas piezelétricas com a estrutura. Esta análise será realizada para o controle passivo e ativo-passivo em uma estrutura tipo placa. Primeiramente é necessário um estudo de diferentes funções densidade de probabilidade, p.d.f. de maneira a garantir uma maior aproximação com o real comportamento da cola em questão. Após a escolha da p.d.f. apropriada um estudo das respostas em frequência da estrutura é realizado observando-se o primeiro modo de vibração para diversas condições de controle e posteriormente uma comparação com outros tipos de p.d.f.será realizada.

\subsection{Estudo de diferentes funções de probabilidade}

Para simplificar a descrição da metodologia adotada para a variável do módulo de Young da cola, faremos $X=E$ para as diferentes funções densidade de probabilidades utilizadas para realizar este estudo. Desta forma um modelo estocástico para cada p.d.f., será construído levando-se em conta as informações disponíveis para $X$ e para manter uma coerência com os problemas físicos, a p.d.f., deve seguir o Princípio da Máxima Entro- 
pia. [45], [40] Este princípio consiste em procurar uma função densidade de probabilidade que maximize a entropia com as informações que são fornecidas pelo problema. Neste desenvolvimento as distribuições terão 5000 indivíduos com dispersão na distribuição próxima de $100 \%$.

\subsubsection{Função densidade de probabilidade Gamma}

Um estudo na função densidade de probabilidade Gamma é realizado, onde devemos lembrar que, como no capítulo 5, a vairável $X$ deve: (1) estar entre $] 0,+\infty[$; (2) a média dos valores são tais que $E[X]=\underline{X}$; e (3) o zero é um valor descartado para variáveis com valores positivos, que é explicado pela condição $E[\ln (X)]=c_{X} \operatorname{com} \mid c_{X}<+\infty$. Desta maneira, o princípio de Máxima Entropia, produz uma função densidade de probabilidade Gamma. [45], [40] , [37], [16]

$$
p_{X}(X)=\mathbb{I}_{] 0,+\infty[}(X) \frac{1}{\underline{X}}\left(\frac{1}{\delta_{X}^{2}}\right)^{\delta_{X}^{-2}} \frac{1}{\Gamma\left(\delta_{X}^{-2}\right)}\left(\frac{X}{\underline{X}}\right)^{\delta_{X}^{-2}-1} \exp \left(-\frac{X}{\delta_{X}^{2} \underline{X}}\right)
$$

observamos que $\delta_{X}=\sigma_{X} / \underline{X}$ é a dispersão relativa de $\widehat{X}$ e $\sigma_{X}$ é o desvio padrão. A função Gamma é definida como $\Gamma(\alpha)=\int_{0}^{\infty} t^{\alpha-1} e^{-t} \mathrm{~d} t$. Para uma análise do módulo de Young podemos observar a p.d.f., representada no histograma da variável aleatória descrita na Figura 6.1.

As variáveis foram obtidas utilizando uma função MATLAB, gamrnd, considerando 5000 realizações e podemos observar que ela segue o comportamento esperado de uma função Gamma, somente com valores positivos e sem picos fora da distribuição. Estes vetores com as realizações aleatórias para a variável $\widehat{X}$, serão aplicadas nas propriedades de entrada do modelo desenvolvido e assim gerar uma nova FRF $G_{p}\left(\theta_{j}, \omega\right), G_{c}\left(\theta_{j}, \omega\right)$ e $G_{h}\left(\theta_{j}, \omega\right)$.

Uma análise preliminar dos valores gerados pela função gamrnd, mostra que de acordo com a Figura 6.1 ocorre uma concentração dos valores do módulo de Young abaixo 


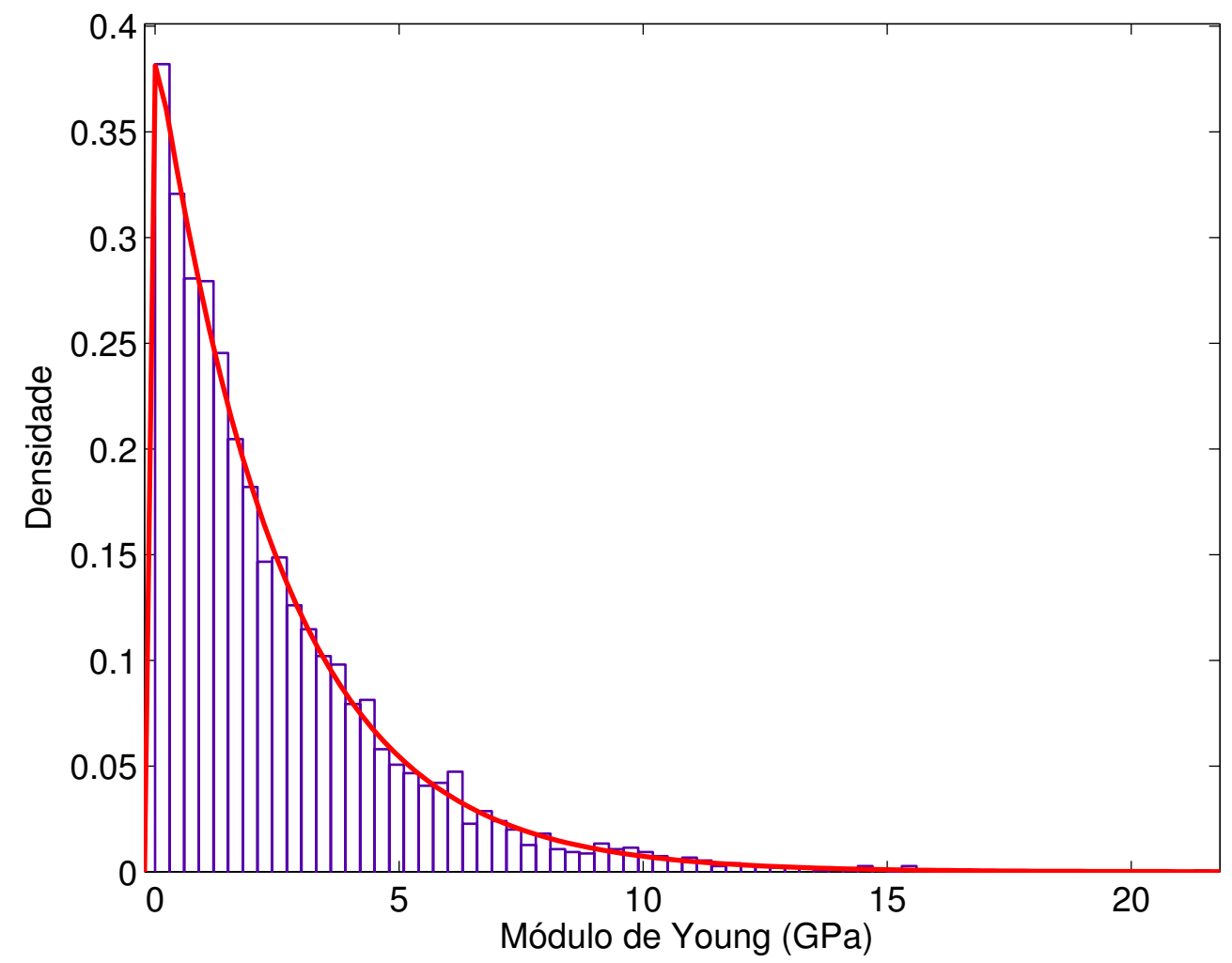

Figura 6.1: Histograma com os valores utilizados para o modelo estocástico através de uma Gamma

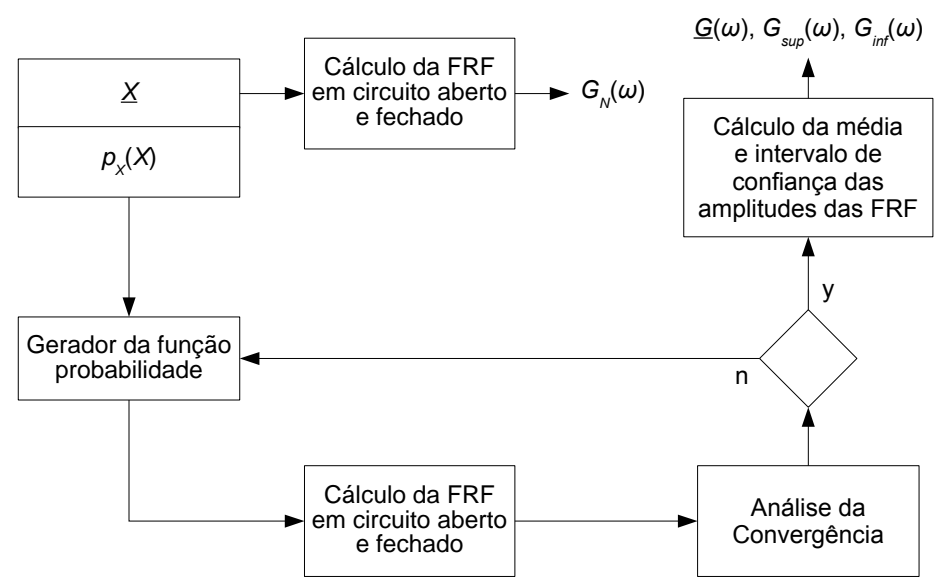

Figura 6.2: Esquema do procedimento computacional para obtenção das respostas em frequência e o calculo do intervalo de confiança para a função densidade de probabilidade Gamma. 
de $2 \mathrm{GPa}$ e esta concentração terá influência direta no comportamento das respostas em frequência da estrutura considerada para o estudo. Este comportamento da distribuição já é um comportamento previsto, porém, como não há muita informação para o módulo de Young da cola procuramos valores de dispersão próximos de 100\% com igual probabilidade. O efeito da concentração dos valores abaixo do valor nominal definido em $2.5 \mathrm{GPa}$ ocasionará uma distorção nas respostas em frequências.

\subsubsection{Função densidade de probabilidade Beta}

Ainda utilizando o Princípio da Máxima Entropia, estudamos a função probabilidade Beta que é compreendida entre os limites $0<X<1$, definida por dois parâmetros livres $\alpha>0$ e $\beta>0$. De maneira que a função probabilidade deve: (1) estar entre $] 0,+1[$; (2) o zero é um valor que descartado para variáveis com valores positivos, que é explicado pela condição $E[\ln (X)]=c_{X} \operatorname{com} \mid c_{X}<+\infty$. Desta maneira, o princípio de Máxima Entropia, produz uma função de probabilidade Beta [41] :

$$
p_{X}(X)=\mathbb{I}_{0,1[}(X) \frac{\Gamma(\alpha+\beta)}{\Gamma(\alpha) \Gamma(\beta)} X^{\alpha-1}(1-X)^{\beta-1}
$$

observamos que a função Gamma é definida como $\Gamma(\alpha)=\int_{0}^{\infty} t^{\alpha-1} e^{-t} \mathrm{~d} t$ e que $\mu_{X}=\frac{\alpha}{\alpha+\beta}$ é a média de $\widehat{X}$ e $\sigma_{X}^{2}=\frac{\alpha \beta}{(\alpha+\beta)^{2}(\alpha+\beta+1)}$ é o variância da função. Para uma análise do conjunto de dados obtidos para o módulo de Young podemos observar a p.d.f., representada no histograma da variável aleatória descrita na Figura 6.3.

Como descrito as variáveis foram obtidas utilizando uma função MATLAB, betarnd, considerando 5000 realizações e podemos observar que ela segue o comportamento esperado de uma função Beta, $\operatorname{com} \alpha=1.2$ e $\beta=1.2$, somente com valores positivos e sem picos fora da distribuição. Estes vetores com as realizações aleatórias para a variável $\widehat{X}$, serão aplicadas nas propriedades de entrada do modelo desenvolvido e assim gerar uma nova resposta em frequência $G_{p}\left(\theta_{j}, \omega\right), G_{c}\left(\theta_{j}, \omega\right)$ e $G_{h}\left(\theta_{j}, \omega\right)$.

Uma análise preliminar dos valores gerados pela função betarnd, mostra que de 


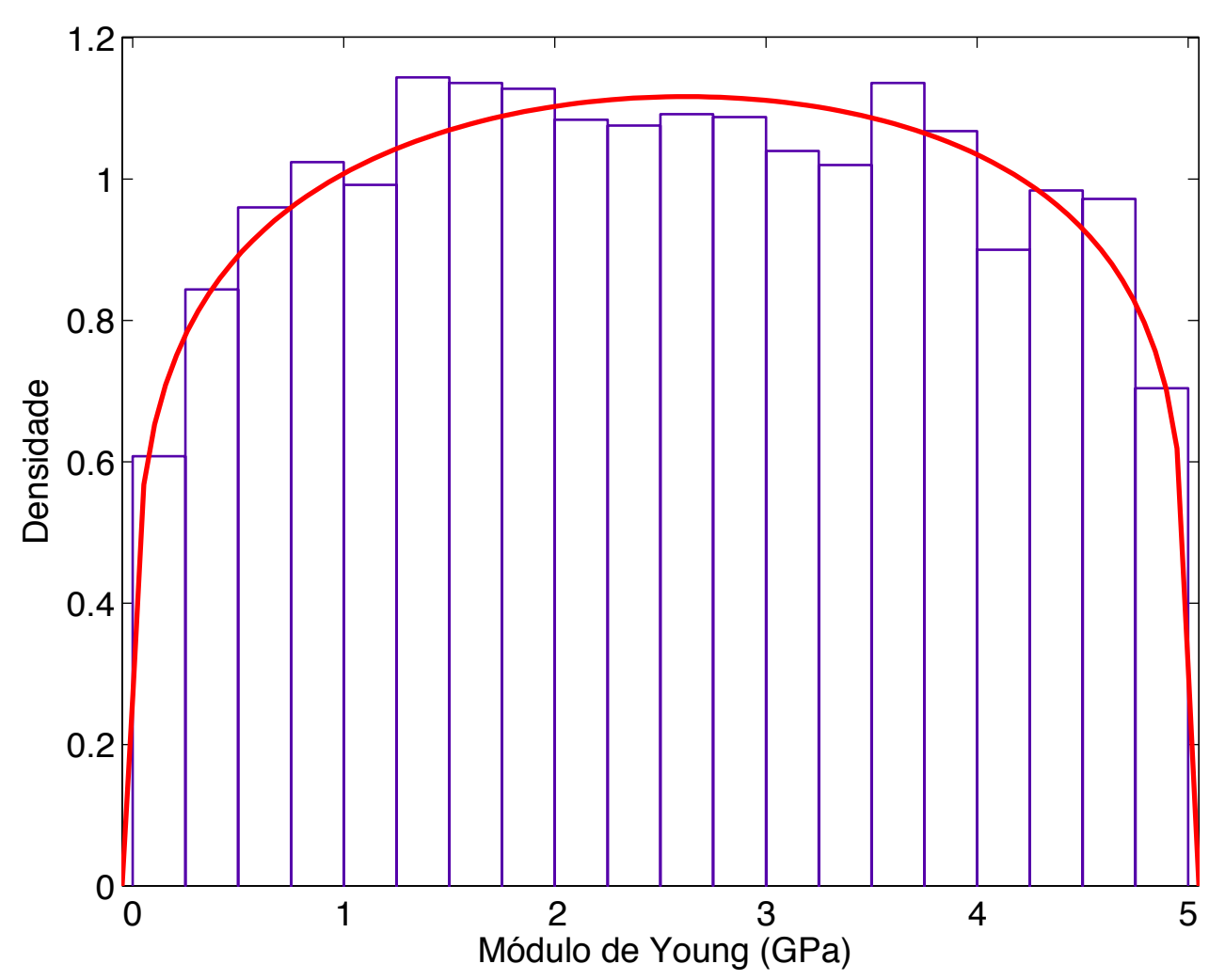

Figura 6.3: Histograma com os valores utilizados para o modelo estocástico através de função densidade de probabilidade Beta

acordo com a Figura 6.3 uma boa distribuição dos valores em torno do valor nominal definido como sendo 2.5 GPa com uma queda abrupta nas extremidades, de acordo com o proposto pela formulação matemática. Porém a dispersão dos valores obtidos foi de no máximo 65\%. Mesmo sendo uma distribuição bem comportada e seguindo o princípio da Máxima Entropia buscaremos uma distribuição que tenha dispersões próximas a $100 \%$.

\subsubsection{Função densidade de probabilidade Gaussiana}

A função distribuição Gaussiana, também chamada de Normal, é uma distribuição estatística contínua com função probabilidade dada por,

$$
p_{X}(X)=\mathbb{I}_{-\infty,+\infty[}(X) \frac{1}{\sigma \sqrt{2 \pi}} e^{\frac{-1}{2}\left(\frac{x-\delta}{\sigma}\right)^{2}}
$$


o parâmetro $\mu$ é a média de $\widehat{X}$, que também é a posição do pico da função gaussiana, e $\sigma$ é o desvio padrão da distribuição. Para uma análise do conjunto de dados obtidos para o módulo de Young podemos observar a p.d.f., representada no histograma da variável aleatória descrita na Figura 6.4.

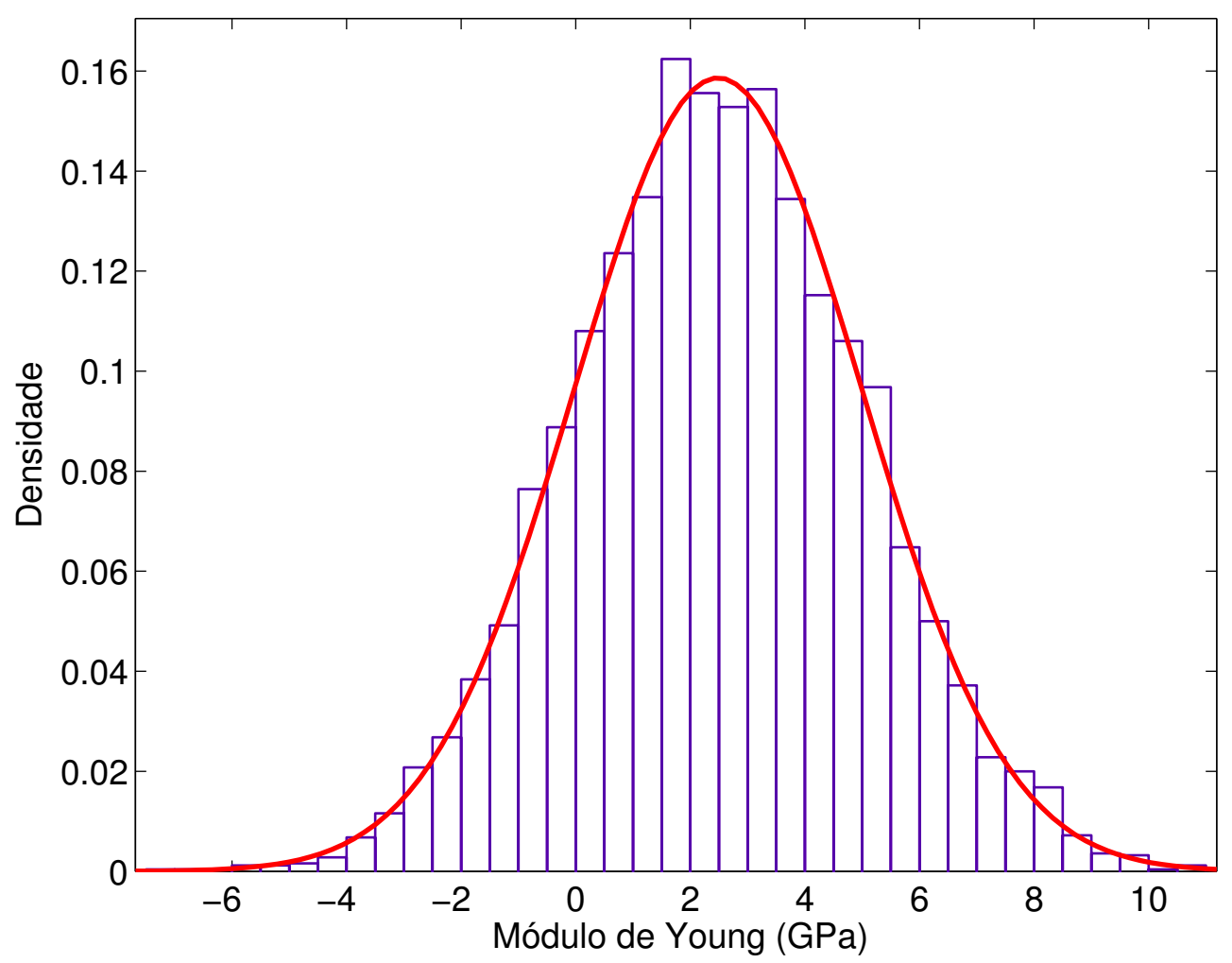

Figura 6.4: Histograma com os valores utilizados para o modelo estocástico através de uma função gaussiana

A distribuição Gaussiana é considerada a função mais utilizada em problemas estatísticos, isto se deve a dois principais motivos: (1) a distribuição Gaussiana é derivada do teorema do limite central, que estabelece que, quando o tamanho da amostra aumenta, a distribuição aproxima-se cada vez mais de uma distribuição normal, sendo esta infinitamente divisível, (2) a distribuição normal é muito tratável analiticamente, isto é, um grande número de resultados envolvem esta distribuição que pode ser derivada de forma 
explicita [1].

Este tipo de função permite valores negativos em sua distribuição e possui a característica de ser simétrica em torno de uma média definida. Desta maneira ela se torna interessante para estudo proposto para o módulo de Young da cola dos elementos piezelétricos na estrutura, pois não conhecemos as reais propriedades da cola e sim somente o seu valor médio, porém para realizar o estudo da influência sobre a estrutura, necessitamos de realizar algumas alterações na distribuição fazendo as seguintes proposições: (1) ser maior que 0 e menor que 5, de forma que ]0,5[; (2) por ser uma distribuição simétrica em torno da média, a média dos valores são tais que $E[X]=\underline{X}$; e (3) o zero é um valor que descartado para variáveis com valores positivos, que é explicado pela condição $E[\ln (X)]=c_{X} \operatorname{com} \mid c_{X}<+\infty$, desta maneira resulta em uma nova distribuição apresentada na Figura 6.5 com uma população menor que os 5000 indivíduos como inicialmente proposto, tendo 4796 indivíduos, com uma dispersão de $94 \%$.

\subsection{Análise dos resultados obtidos para estrutura tipo placa $500 \times 400$ com cola}

Nesta seção analisaremos os resultados obtidos através das realizações para uma estrutura com as dimensões de uma estrutura tipo placa com $500 \mathrm{~mm}$ de comprimento e $400 \mathrm{~mm}$ de largura sendo os elementos piezelétricos acoplados em sua superfície através de uma camada de cola de $0.1 \mathrm{~mm}$ de espessura em toda área em contato entre as pastilhas piezelétricas e a placa, localizado a $5 \mathrm{~mm}$ dos lados engastados.

Nesta configuração, consideraremos cerâmicas piezelétricas feitas de PZT-5H e com as mesmas propriedades utilizadas no capítulo anterior. Para os componentes do circuito elétrico foram considerados os valores de resistências e indutâncias, otimizados através do processo de algoritmo genético como detalhado na seção anterior, sendo $R_{c}=1.2055 \mathrm{k} \Omega$ e $L_{c}=24.8557 \mathrm{H}$ para o caso passivo otimizado e $R_{c}=1.1129 \mathrm{k} \Omega \mathrm{e}$ 


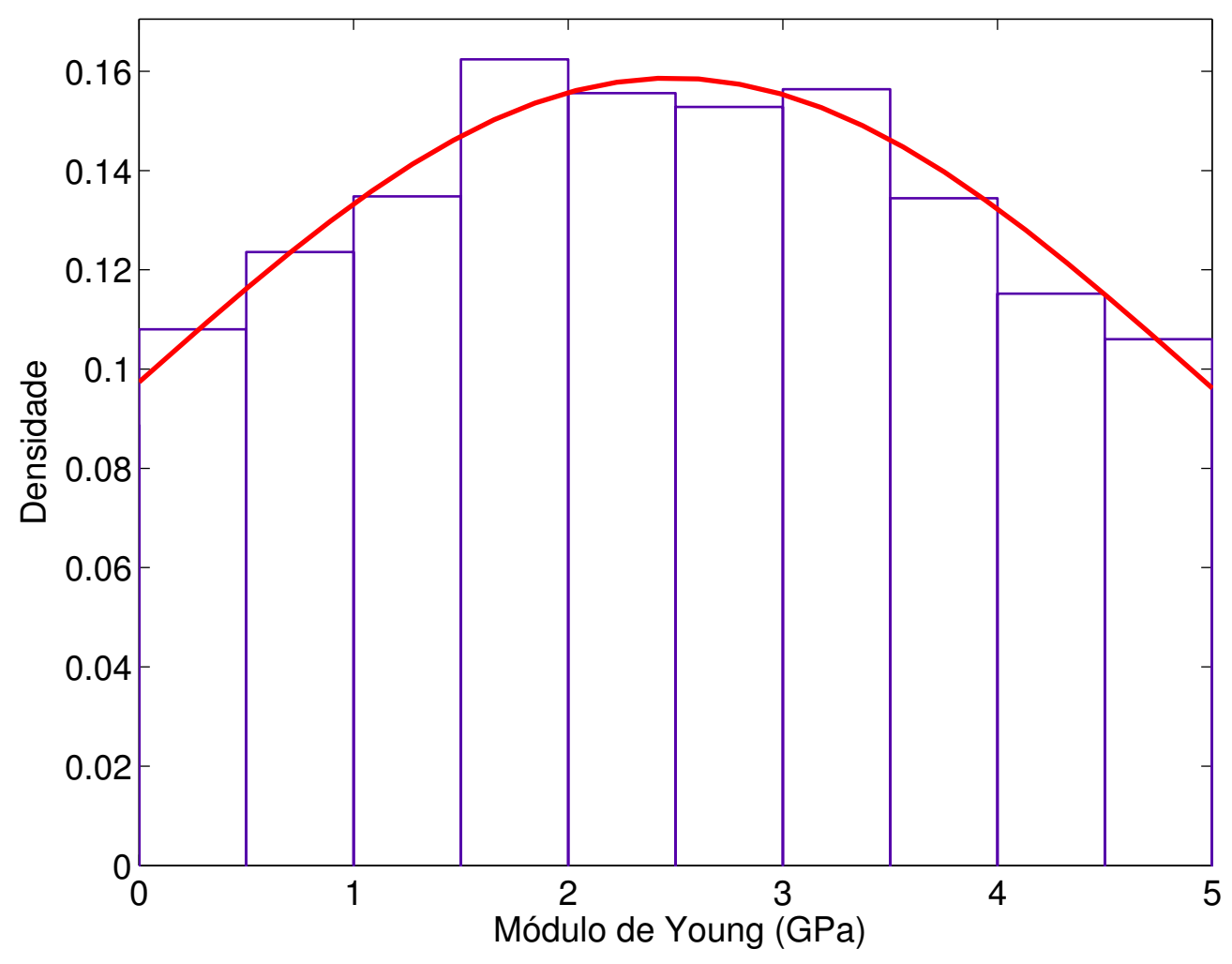

Figura 6.5: Histograma com os valores utilizados para o modelo estocástico através de uma função densidade de probabilidade gaussiana truncada

$L_{c}=24.7410 \mathrm{H}$ para o caso ativo-passivo otimizado para $\mathrm{V}=250 \mathrm{~V}$.

Uma análise realizada considerando a configuração em extensão da estrutura proposta, pode ser vista na Figuras 6.7 e 6.8, onde a resposta em circuito aberto (OC) $\left(G_{p}^{O C}\right) \mathrm{e}$ conectada a um circuito shunt otimizado $\left(G_{p}^{N}\right)$ pode ser observado, lembrando que foram utilizadas as 4796 realizações variando somente o módulo de Young para gerar o intervalo de confiança de $95 \%$ para todas as respostas e distribuições.

Analisando as respostas para as incertezas do módulo de Young, figura 6.7, o intervalo de confiança mostrou uma redução de $17 \mathrm{~dB}$ com o valor médio deste intervalo de confiança quando comparado a estrutura em circuito aberto e de $13.5 \mathrm{~dB}$ para o limite superior do intervalo de confiança quando comparado a estrutura em circuito aberto. 

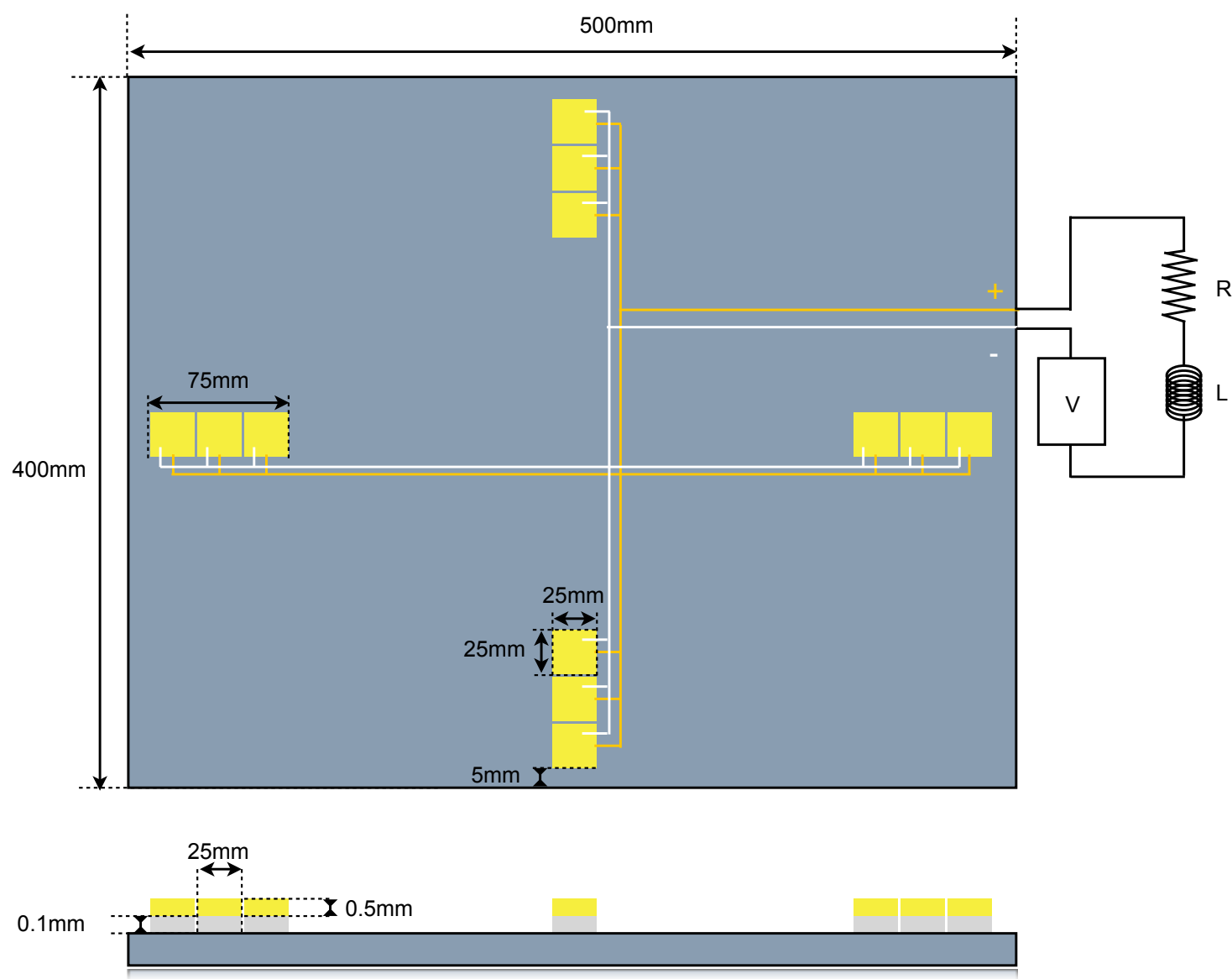

Figura 6.6: Esquema da estrutura tipo placa utilizada para análise do efeito da cola entre os elementos piezelétricos e estrutura, com circuito elétrico conectado nas pastilhas piezelétricas.

Realizou-se uma análise da influência do controle ativo-passivo para este caso, onde observou-se que, como apresentado pela Figuras 6.9 e 6.10 com ganho constante, a redução entre a estrutura em circuito aberto e a média do intervalo de confiança foi de $19 d B$ e a redução da estrutura em circuito aberto em relação ao limite superior do intervalo de confiança foi de $14 d B$.

No caso apresentado pela Figura 6.11 e 6.10, que considera ganho variável, a redução entre a estrutura em circuito aberto e a média do intervalo de confiança foi de $19 d B$ e a redução da estrutura em circuito aberto em relação ao limite superior do intervalo de confiança foi de $17 d B$. 


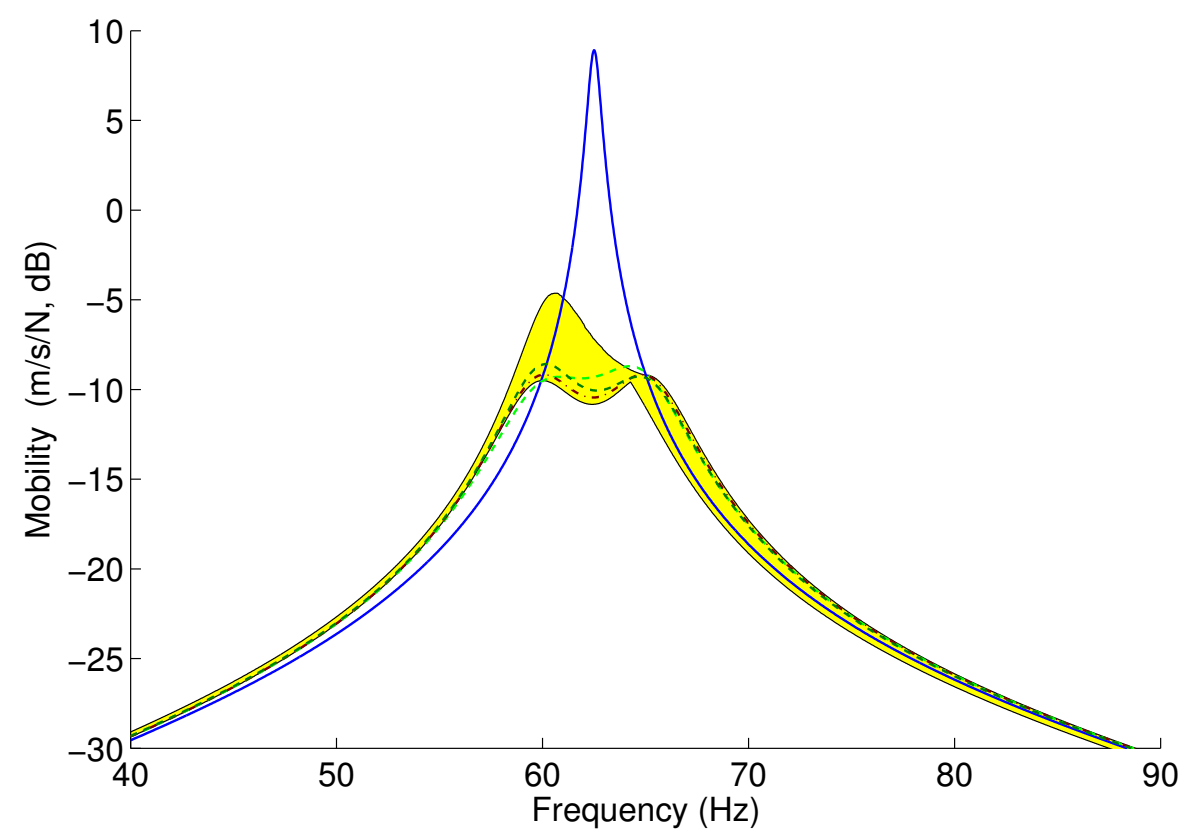

Figura 6.7: Intervalo de confiança para análise estocástica para o primeiro modo com incerteza na cola, acoplado a um circuito elétrico controle puramente passivo.

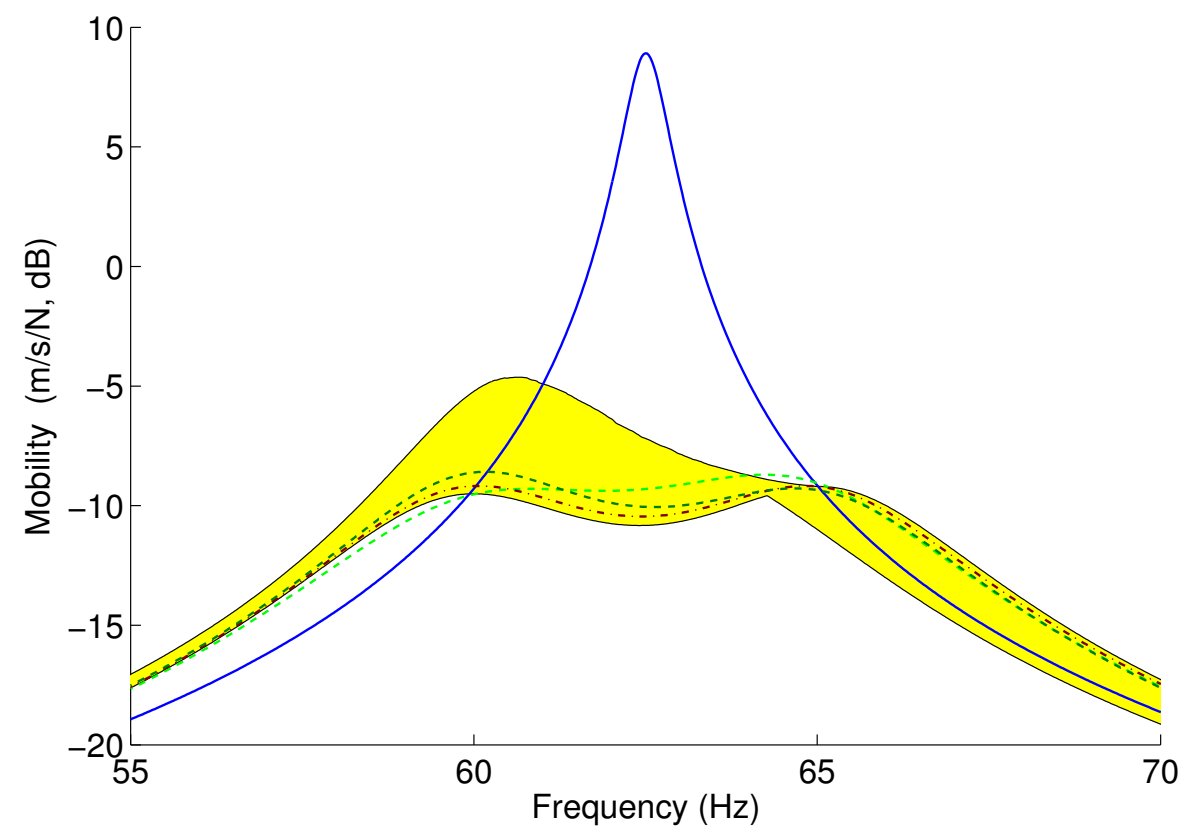

Figura 6.8: Intervalo de confiança para análise estocástica para o primeiro modo com incerteza na cola acoplado a um circuito elétrico controle puramente passivo [zoom]. 


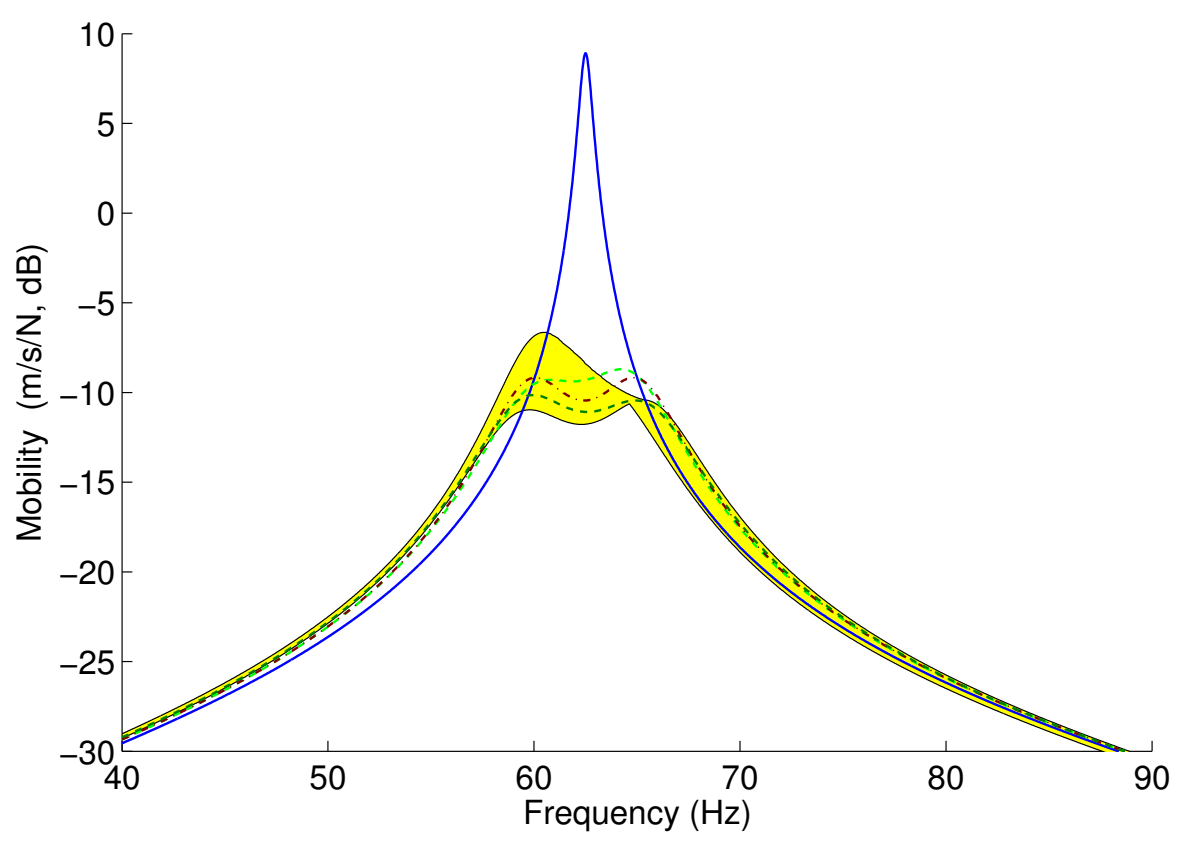

Figura 6.9: Intervalo de confiança para análise estocástica para o primeiro modo com incerteza na cola acoplado a um circuito elétrico controle ativo-passivo com ganho constante.

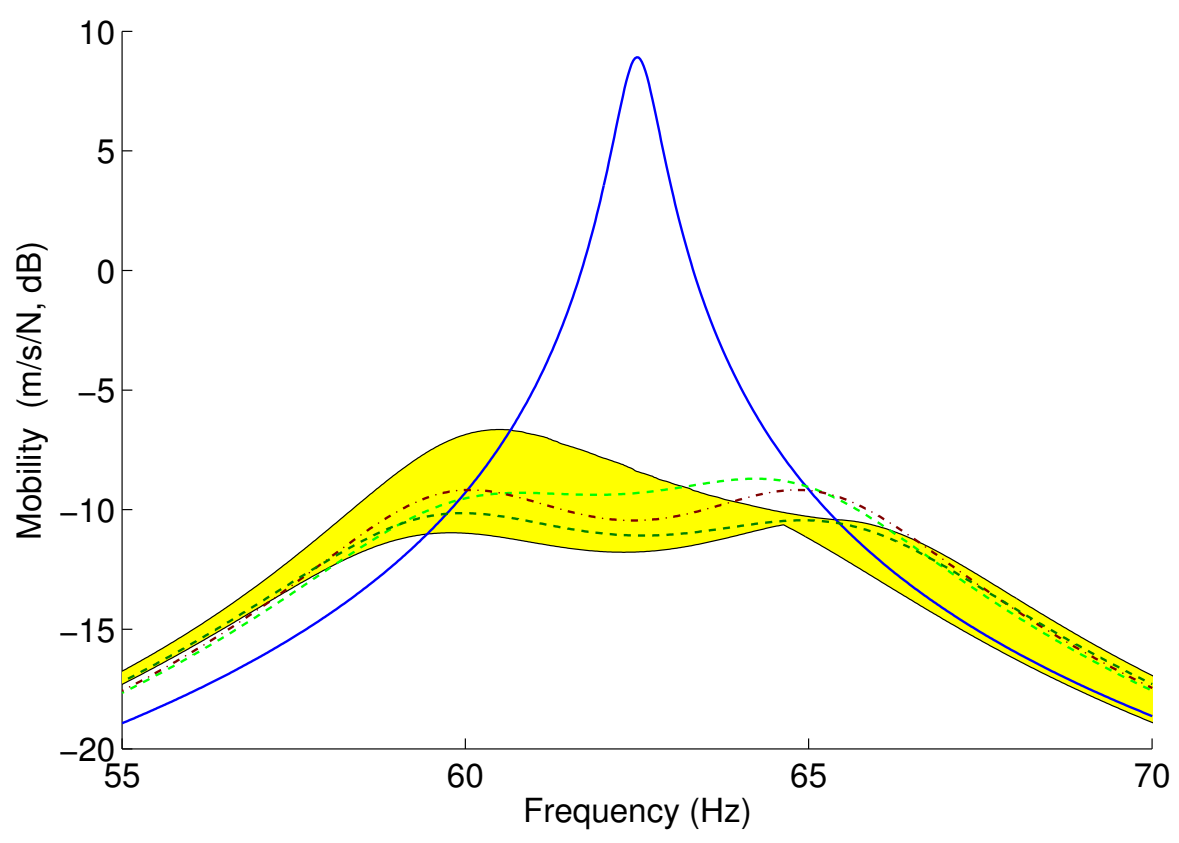

Figura 6.10: Intervalo de confiança para análise estocástica para o primeiro modo com incerteza na cola acoplado a um circuito elétrico controle ativo-passivo com ganho variável [zoom]. 


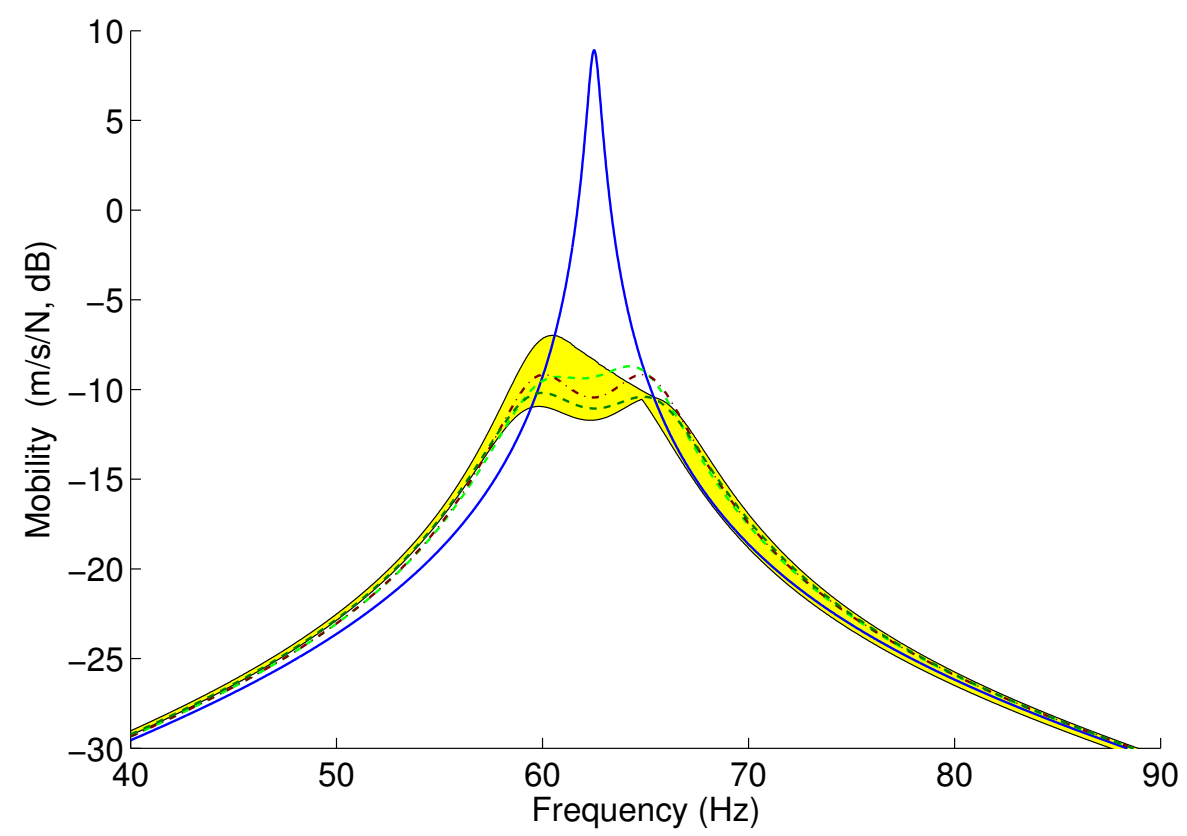

Figura 6.11: Intervalo de confiança para análise estocástica para o primeiro modo com incerteza na cola acoplado a um circuito elétrico controle ativo-passivo com ganho variável.

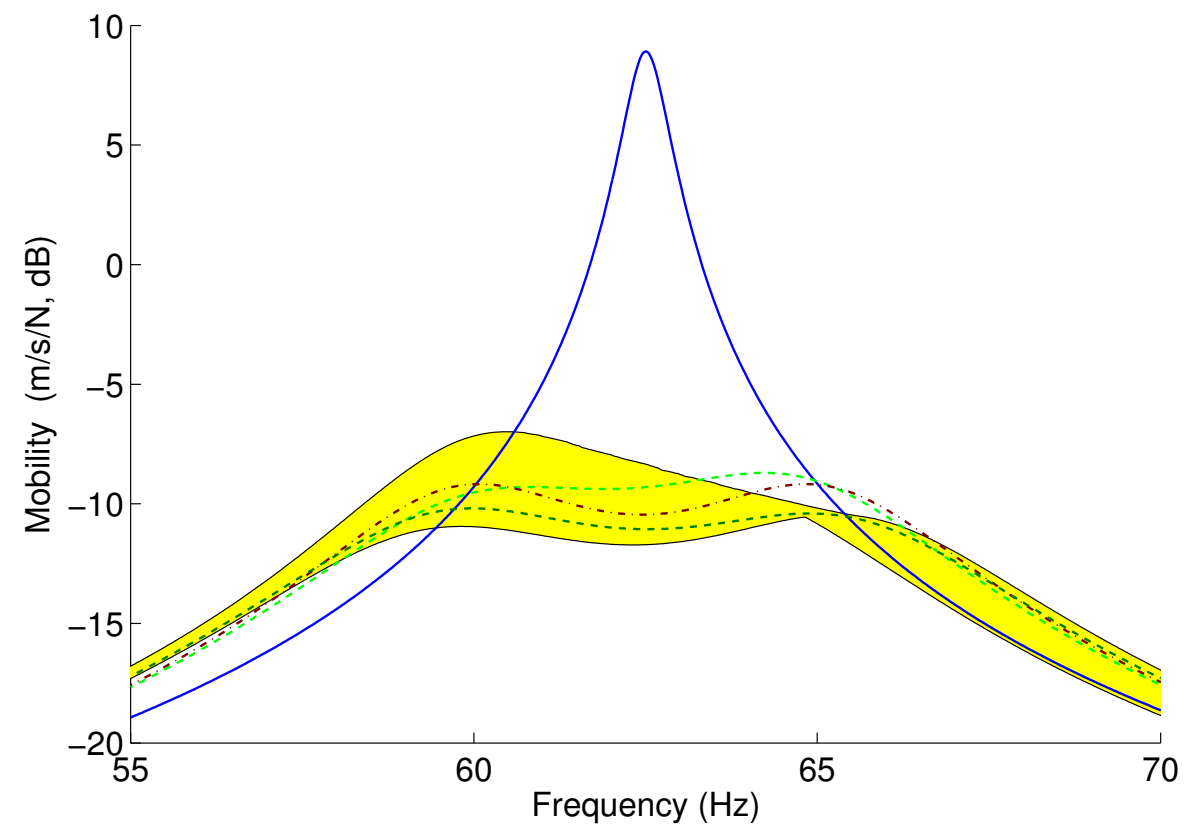

Figura 6.12: Intervalo de confiança para análise estocástica para o primeiro modo com incerteza na cola acoplado a um circuito elétrico controle ativo-passivo com ganho variável [zoom]. 


\subsection{Comparação}

Nesta seção ilustramos uma comparação do comportamento das diversas distribuições para a variável do módulo de Young, sobre a resposta em frequência e seu intervalo de confiança.

Analisando a Figura 6.13(a), observamos o primeiro modo de vibração da estrutura proposta utilizando a p.d.f. Gamma, dada pela Figura 6.1. Nesta situação o intervalo de confiança apresenta um deslocamento para esquerda, indicando que a p.d.f. gamma, apesar de ter valor médio igual ao nominal, 2.5 GPa, e dispersão de 100\%, possui majoritariamente valores abaixo do valor nominal. Uma comparação com a estrutura em circuito aberto, a reducão de $10 \mathrm{~dB}$ comparado com o limite superior do intervalo de confiança e $17 \mathrm{~dB}$ com o valor médio deste intervalo.

Realizando uma análise na Figura 6.13(b), que utiliza a p.d.f. Beta, dada pela Figura 6.3, observamos que o intervalo de confiança é menor que os outros casos. Este fato se deve a dispersão da p.d.f. Beta ser menor que as outras distribuições, mostrando que a dispersão influência diretamente na resposta. Nesta situação observa-se uma redução de $15.5 \mathrm{~dB}$ em comparação ao limite superior do intervalo de confiança e a estrutura em circuito aberto e $18 \mathrm{~dB}$ em relação ao valor médio desse mesmo intervalo. 

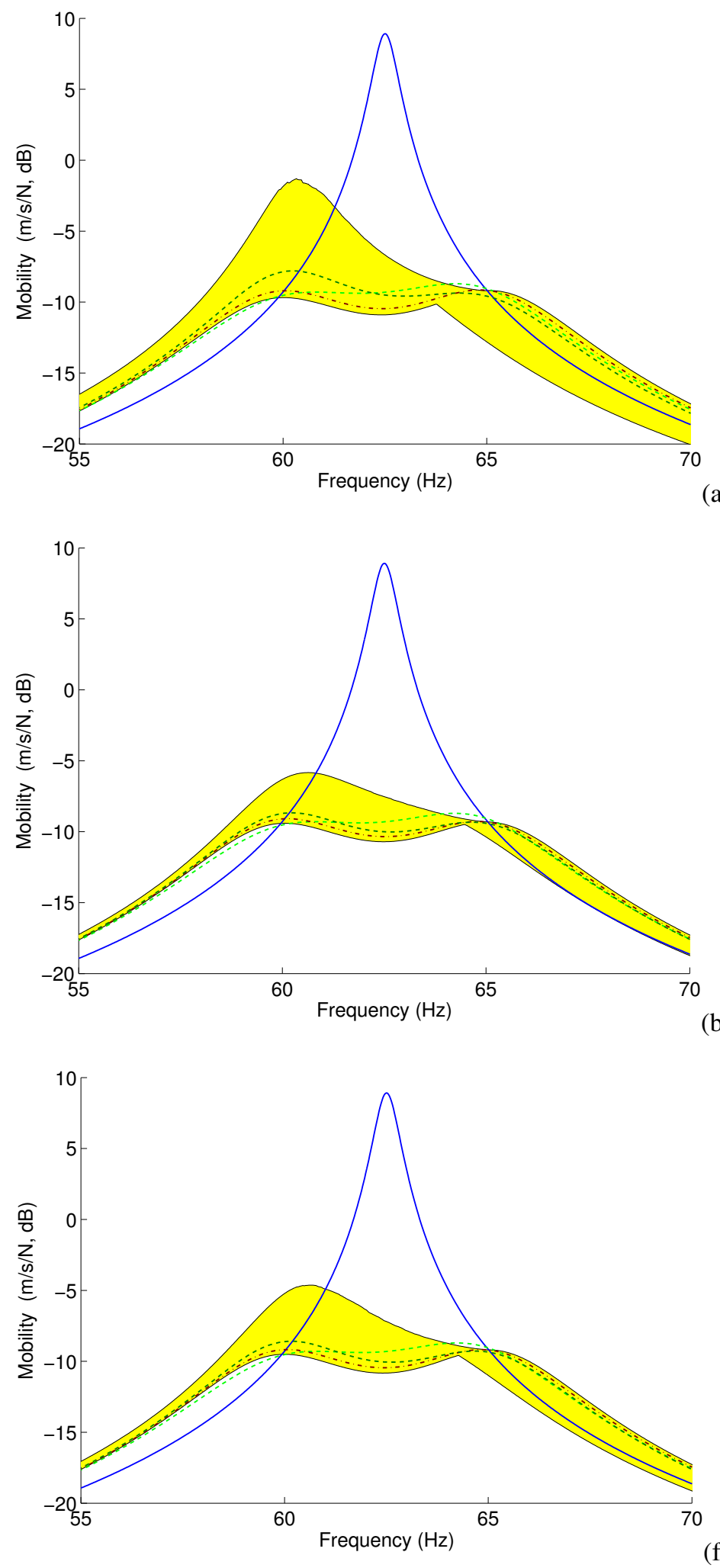

Figura 6.13: Comparação das diferentes respostas em frequência para o primeiro modo de vibração na mesma estrutura utilizando a distribuição Gamma (a), distribuição Beta (b) e distribuição Gaussiana truncada. 


\section{Capítulo 7}

\section{Conclusões}

Esta tese apresentou uma análise numérica do controle de vibrações estruturais através de cerâmica piezoelétricas em extensão conectadas a circuitos ativo-passivos compostos por resistência, indutância e fonte de tensão. Para tal um modelo de elementos finitos de placa sanduiche com três camadas elásticas e/ou piezoelétricas foi desenvolvido. Realizou-se também uma modelagem dos componentes do circuito elétrico e seu acoplamento à estrutura gerando assim uma equação de movimento acoplada para a estrutura com elementos piezoelétricos conectados aos circuitos elétricos.

Uma análise harmônica das equações obtidas foi realizada para se obter uma avaliação preliminar dos efeitos causados pelos componentes elétricos do circuito na estrutura. Observou-se que os elementos passivos do circuito, resistência e indutância, tem não somente um efeito de absorvedor dinâmico de vibrações mas, também, promovem uma amplificação da autoridade de controle no caso de se atuar através da fonte de tensão.

A metodologia tradicional de projeto de absorvedores dinâmicos de vibrações foi utilizada para derivar expressões para os valores de resistência e indutância de modo a maximizar o desempenho passivo do sistema. Uma análise numérica do desempenho na redução das amplitudes de vibração em uma estrutura tipo placa/viga engastada-livre com um elemento piezoelétrico próximo ao seu engaste mostrando que, o modelo e o controle, apresentaram bons resultados controlando o comportamento da estrutura com reduções 
de $20 \mathrm{~dB}$ com o circuito ativo-passivo.

Com a inclusão da fonte de tensão e uma lei de controle LQR interativa, a qual leva em consideração uma limitação de tensão aplicada, um estudo de controle híbrido ativo-passivo da mesma estrutura foi realizado e observou-se que obtem-se uma redução na amplitude de $25 \mathrm{~dB}$.

Com um modelo válido e funcional, estudou-se o comportamento das incertezas que compreendem o projeto, como a resistência e a indutância do circuito elétrico, bem como as propriedades dielétricos das cerâmicas piezoelétricas. Para isto foi utilizado uma metodologia de processos estocásticos, o qual através de definições estatísticas com significado físico, como o principio de Máxima Entropia, podemos definir um conjunto de valores estatísticos para cada variável através de um percentual de dispersão, sempre mantendo uma média e um desvio padrão em torno dos valores nominais.

Observou-se que para uma dispersão de $10 \%$ em torno do valor nominal do parâmetro, o componente do circuito elétrico responsável por maior distorção na amplitude da resposta em frequência da estrutura é a indutância, ocasionando um desajuste no circuito elétrico e consequentemente desajuste no controle proposto. Uma redução de 28 para $10 \mathrm{~dB}$ foi observada comparando-se a estrutura em circuito aberto e o limite superior do intervalo de confiança.

A análise das incertezas na resistência elétrica, que compõe o circuito elétrico, ocasiona um aumento ou diminuição no nível da amplitude de vibração da estrutura, este fenômeno já era esperado pois a função da resistência é de reduzir a amplitude da vibração. Uma redução de 24.8 dB entre a estrutura em circuito aberto e o limite superior do intervalo de confiança foi observado.

A mesma dispersão para os valores das propriedades dielétricas das cerâmicas piezoelétricas foi considerado e verificou-se que analogamente ao circuito a propriedade piezoelétrica que ocasiona maior desajuste é $\beta_{33}$. Uma redução de $23.3 \mathrm{~dB}$ foi observada entre o limite superior do intervalo de confiança e a estrutura em circuito aberto. 
Analogamente a mesma análise foi realizada para as propriedades dielétricas do material piezelétrico apresentando uma redução de $22.2 \mathrm{~dB}$ quando comparado o limite superior do intervalo de confiança com a estrutura em circuito aberto.

Uma análise da autoridade de controle em estruturas piezelétricas utilizando controle ativo-passivo possibilitou uma mudança na estrutura a ser desenvolvida, bem como a introdução de uma metodologia de otimização, chamada algoritmo genético. A otimização proposta foi para valores da resistência e indutância do circuito elétrico que compõe o controle ativo-passivo.

Este processo de otimização buscou através de uma função objetivo, minimizar a amplitude da resposta em frequência da estrutura em um intervalo de frequência, para vários valores de amplitude de força de entrada. Este processo de otimização, mostrou o comportamento da autoridade de controle para diferentes voltagens e diferentes amplitudes de força de entrada. Esta análise demonstrou que a autoridade de controle aumenta quando a força de amplitude diminui sendo prioritariamente ativo o controle a ser aplicado na estrutura.

Com os valores de resistência e indutância otimizados, um estudo do efeito das incertezas nas propriedades da camada de cola que conecta o material piezelétrico com a estrutura metálica. Procurando uma dispersão grande para as propriedades do material que compõe a cola, $100 \%$, foi necessário um estudo de diferentes funções probabilidade.

Funções de probabilidade Gamma demonstraram uma concentração dos valores de módulo de Young da cola em torno de valores de $2 \mathrm{GPa}$, não demonstrando uma distribuição homogênea em torno do valor nominal, como esperado por se tratar de uma função Gamma. O efeito da distrubuição Gamma, na incerteza do módulo de Young da cola, ocasionou uma deformação do intervalo de confiança.

Funções de probabilidade Beta demonstrarm uma boa distribuição em torno do valor nominal, porem, a dispersão utilizando esta função não foi satisfatória para nosso estudo, onde se procurava dispersões de $100 \%$. 
Desta forma procurou-se uma distribuição Gaussiana, que é bem comportada, porem, permite valores negativos, fato inviável ao se analisar o módulo de Young de um material. Um tratamento dos dados foi necessário em posteriori de tal forma a manter os valores sempre positivos, inferiores ao dobro do valor nominal e com valor médio igual ao nominal. Os resultados mostram uma redução de $17 \mathrm{~dB}$ para incertezas ligadas ao módulo de Young da cola na estrutura para a configuração proposta. Uma comparação dos diversos resultados foi realizada.

Procurando a continuidade do trabalho desenvolvido até o momento, propõe-se a otimização dos parâmetros geométricos e das posições das pastilhas, procura de novas configurações que promovam a controlabilidade de diversos modos, um estudo em conjunto das incertezas do controle e das propriedades da cola para a perda da autoridade de controle e a utilização de outras técnicas para a lei de controle considerada. 


\section{Referências Bibliográficas}

[1] Abramovich, Y. A., 2002, "An invitation to operator theory," American Mathematical Society., Stanford, United States do America.

[2] Ahmadian, M. e DeGuilio, A., 2001, "Recent advances in the use of piezoceramics for vibration suppression," The Shock and Vibration Digest, Vol. 33, No. 1, pp.1522.

[3] Allik, H. and T. J. R. Hughes, 1970, "Finite Element Method for Piezoelectric Vibration, " International Journal for Numerical Methods in Engineering, 2:151-157.

[4] Back, T., U. Hammel e H. P. Schwefel, 1997, "Evolutionary Computation: Comments on the History and Current State.," IEEE Transactions On Evolutionary Computation, Vol. 1, No. 1, pp. 3-17.

[5] Bailey, T. e Hubbard, J.E.J., 1985, "Distributed piezoelectric-polymer active vibration control of a cantilever beam," AIAA Journal, Vol. 8, No. 5, pp.605-611.

[6] Baillargeon, B.P. ; Vel, S. 2005. "Exact solution for the vibration and active damping of composite plates with piezoelectric shear actuators.” Journal of Sound and Vibration, v.282, p.781-804.

[7] Baillargeon, B.P. and Vel, S.S., 2005, "Active vibration suppression of sandwich beams using piezoelectric shear actuators: experiments and numerical simulations," Journal of Intelligent Materials Systems and Structures, Vol. 16, No. 6, pp.517-530. 
[8] Benjeddou, A., 2000, "Advances in piezoelectric finite element modeling of adaptive structural elements: a survey," Computers and Structures, Vol. 76, pp.347-363.

[9] Benjeddou, A., Trindade, M.A., and Ohayon, R., 1997, “A Unified Beam Finite Element Model for Extension and Shear Piezoelectric Actuation Mechanics," Journal of Intelligent Materials Systems and Structures, Vol. 8, No. 3, pp.1012-1025.

[10] Benjeddou, A., 2007, "Shear-mode piezoceramic advanced materials and structures: a state of the art," Mechanics of Advanced Materials and Structures, Vol. 14, pp. 263-275.

[11] Benjeddou, A. and Ranger-Vieillard, J.-A., 2004, "Passive vibration damping using shunted shear-mode piezoceramics," In Topping, B.H.V. and Mota Soares, C.A., eds., Proceedings of the Seventh International Conference on Computational Structures Technology, Civil-Comp Press, Stirling, Scotland, p.4.

[12] Benjeddou, A., Trindade, M.A., and Ohayon, R., 1999, "New shear actuated smart structure beam finite element," AIAA Journal, Vol. 37, No. 3, pp.378-383.

[13] Benjeddou, A., Ranger, J.A., 2006, "Use of shunted shear-mode piezoceramics for structural vibration passive damping," Computers and Structures, Vol. 84, pp.14151425.

[14] Benjeddou, A., Ranger, J.A., 2006, "Vibration Damping Using Resonant Shunted Shear-Mode Piezoceramics," III European Conference on Computational Mechanics Solids, Structures and Coupled Problems in Engineering, .

[15] Benjeddou, A., Ranger-Vieillard, J.A., 2004, "Passive Vibration Damping using Shunted Shear-Mode Piezoceramics," Proceedings of the Seventh International Conference on Computacional Structures Technology, paper 4. 
[16] Cataldo, E., Soize, C., Sampaio, R. and Desceliers, C., 2009, "Probabilistic modeling of a nonlinear dynamical system used for producing voice. ", Computacional Mechanics, Vol. 43, pp 265-275.

[17] Chopra, I., 2002, "Review of State of Art of Smart Structures and Integrated Systems," AIAA Journal, Vol. 40, No. 11, pp.2145-2179.

[18] Crawley, E.F., e Luis, J., 1987, “Use of piezoelectric actuators as elements of intelligent structures," AIAA Journal, Vol. 25, No. 10, pp.1373-1385.

[19] Deü, J.F. ; Benjeddou, A. 2005. "Free-vibration analysis of laminated plates with em- bedded shear-mode piezoceramic.” International Journal of Solids and Structures, v.42, p.2059-2088.

[20] Den Hartog, J.P., 1972, "Vibrações nos Sistemas Mecânicos,” Editora Edgard Blücher Ltda., São Paulo, Brasil.

[21] Dosch, J.J., Inman, D., e Garcia, Ephrahim, 1992, “A self-sensing piezoelectric actuator for collocated control,' Journal of Intelligent Material System and Structures, Vol. 3, pp.166-185.

[22] Durand, J. and Soize, C., 2008, "Structural-acoustic modeling of automotive vehicles in presence of uncertainties and experimental identification and validation.", Journal Acoustic Society American, Vol. 124, pp-1513-1525.

[23] Forward, R.L., 1979, "Electronic damping of vibrations in optical structures," Applied Optics, Vol. 18, No. 5, pp.690-697.

[24] Gopinathan, S.V. ; Varadan, V.V. ; Varadan, V.K. (2000). "A review and critique of theories for piezoelectric laminates." Smart Materials and Structures, v.9, p.24- 48. 
[25] Hagood, N.W. and von Flotow, A., 1991, "Damping of structural vibrations with piezoelectric materials and passive electrical networks," Journal of Sound and Vibration, Vol. 146, No. 2, pp.243-268.

[26] Harertling, G.H., 1999, "Ferroelectric Ceramics: History and Technology," Journal American Ceramic Society, Vol. 82, No. 4, pp.797-818.

[27] Ikeda, T., 1997, "Fundamentals of Piezoelectricity", Oxford University Press, New York, USA.

[28] Jiang, L.J., Tang, J., and Wang, K.W., 2006, “An enhanced frequency-shift-based damage identification method using tunable piezoelectric transducer circuitry," Smart Materials and Structures, Vol. 15 , pp.799-808.

[29] The Institute of Electrical and Electronics Engineers Inc,, 1987, "IEEE Standard on Piezoelectricity" n. 176-1987.

[30] Khdeir, A.A. ; Aldraihem, O. J. 2007. "Analytical models and solutions of laminated composite piezoelectric plates.” Mechanics of Advanced Materials and Structures, v.14, p.67-80.

[31] Kree, P. and Soize, C. 1983, "Méchanique Aléatoire.” Book, pp 644

[32] Lesieutre, G.A., 1998, "Vibration damping and control using shunted piezoelectric materials," The Shock and Vibration Digest, Vol. 30, No. 3, pp.187-195.

[33] Michaelwicz, Z. e R. Hinterding., 1997, "Evolutionary Algorithms.”, Kluwer Academic.

[34] Moheimani, S.O., 2003, "A Survey of Recent Innovations in Vibration Damping and Control Using Shunted Piezoelectric Transducers," IEEE Transactions on Control Systems Technology, Vol. 11, No. 4, pp.482-494. 
[35] Morgan, R.A., and Wang, K.W., 2002, “An Active-Passive Piezoelectric Absorber for Structural Vibration Control Under Harmonic Excitations With Time-Varying Frequency, Part 1: Algorithm Development and Analaysis," Journal of Vibration and Acoustics, Vol. 124, pp.77-83.

[36] “Otimização de Estruturas com Multi-Objetivos via Algoritmos Genéticos”. 2001. 206 p. Tese (Doutorado) - COOPE, Universidade Federal do Rio de Janeiro, Rio de Janeiro, 2001.

[37] Pandit, M.K., Singh, B.N. and Sheikh, A.H., 2009, "Stochastic perturbation-based finite element for deflections statistics of soft core sandwich plate with random material properties." International Journal of Mechanical Sciences, Vol. 51, pp 363 371.

[38] Raja, S., Prathap, G., and Sinha, P.K., 2002, "Active vibration control of composite sandwich beams with piezoelectric extension-bending and shear actuators," Smart Materials and Structures, Vol. 11, No. 1, pp.63-71.

[39] Reddy, J.N. (1999). "On laminated composite plates with integrated sensors and actuators.” Engineering Structures, v.21, p.568-593.

[40] Ritto, T.G., Soize, C., Sampaio, R., 2010. "Probabilistic model indentification of the bit-rock-interation-model uncertainties in nonlinear dynamics of a drill-string", Mechanis Research Communications, Vol 37, pp 584-589.

[41] Ritto, T.G., Soize, C., Sampaio, R., 2010. "Stochastic Dynamics of a Drill-String with Uncertain Weight-on-Hook", Journal of the Brazilian Society of Mech. Sci \& Eng., Vol XXXII, pp 250-258.

[42] Santos, H. F. L. 2007. "Controle de vibrações estruturais usando cerâmica piezoelétricas em extensão e cisalhamento conectadas a circuitos híbridos ativo- 
passivos” Dissertação de Mestrado, Escola de Engenharia de São Carlos - Universidade de São Paulo (EESC-USP).

[43] Saravanos, D. A. ; Heyliger, P.R.; Hopkins, D.A. 1997. "Layerwise mechanics and finite element for the dynamic analysis of piezoelectric composite plates." International Journal of Solids Structures, v.34, n.3, p.359-378.

[44] Sett, K., Jeremic, B. and Levent Kavvas, M., 2011, "Stochastic elastic-plastic finite elements.”, Computacional Methods Applied Mechanical Engeenering. Vol 200, pp 997-1007.

[45] Soize, C. and Batou, A., 2008, "Identification of Stochastic Loads Applied to a Nonlinear Dynamical System Using and Uncertain Computational Model," Mathematical Problems in Engineering, Vol. 2008.

[46] Soize, C., and Chebil, H., 2003, "Random Uncertainties Model in Dynamic Substructuring Using a Nonparametric Probabilistic Model.” , Journal of Engineering Mechanics. Vol. 129, pp 449-453.

[47] Soize, C., 2000, "Maximum entropy approach for modeling random uncertainties in transient elastodynamics." , Journal Acoustic Society American, Vol 2001, pp 1979-1983.

[48] Soize, C., Capiez-Lernout, E., Durand, J.F., Fernandez, C. and Gagliardini, L., 2008, "Probabilistic model identification of uncertainties in computacional models for dynamical systems and experimental validation., Vol. 198, pp 150-163.

[49] Sunar, M. e Rao, S.S., 1999, "Recent advances in sensing and control of flexible structures via piezoelectric materials technology," Appl Mech Rev, Vol. 52, No. 1, pp.1-16.

[50] Sun, C.T. and Zhang, X.D., 1995, "Use of thickness-shear mode in adaptive sandwich structures," Smart Materials and Structures, Vol. 4, No. 3, pp.202-206. 
[51] Sirohi, J. e Chopra, I., 2001, "Actuator power reduction using L-C oscillator circuits," Journal of Intelligent Material Systems and Structures, Vol. 12, pp.867-877.

[52] Tang, J., Liu, Y., and Wang, K.W., 2000, "Semiactive and Active-Passive Hybrid Structural Damping Treatments via Piezoelectric Materials," The Shock and Vibration Digest, Vol. 32, No. 3, pp.189-200.

[53] Tang, J., e Wang, K.W., 2004, "Vibration Confinement via Optimal Eigenvector Assignment and Piezoelectric Networks," Journal of Vibration and Acoustics, Vol. 126, pp.27-36.

[54] Tang, J., e Wang, K.W., 2001, "Active-passive hybrid piezoelectric networks for vibration control: comparisons and improvement," Smart Materials and Structures, Vol. 10, pp.794-806.

[55] Tsai, M.S., e Wang, K.W., 1999, “On the structural damping characteristics of active piezoelectric actuators with passive shunt," Jounal of Sound and Vibration, Vol. 221, No. 1, pp.1-22.

[56] Thornburgh, R.P., Chattopadhyay, A., and Ghoshal, A., 2004, "Transient Vibration of Smart Structures Using a Coupled Piezoelectric-Mechanical Theory," Journal fo Sound and Vibration, Vol. 274, pp.53-72.

[57] Thornburgh, R.P., and Chattopadhyay, A., 2002, "Simultaneous Modeling of Mechanical and Electrical Response of Smart Composite Structures," AIAA Journal, Vol. 40, No. 8, pp.1603-1610.

[58] Thornburgh, R.P., and Chattopadhyay, A., 2003, "Modeling and optimization of passively damped adaptive composite structures," Journal of Intelligent Materials Systems and Structures, Vol. 14, No. 4-5, pp.247-256. 
[59] Trindade, M.A., 2007, "Simultaneous extension and shear piezoelectric actuation for active vibration control of sandwich beams," Journal of Intellingent Material Systems and Structures, Vol. 18, pp.591-600.

[60] Trindade, M.A., Benjeddou, A., and Ohayon, R., 2001, "Piezoelectric Active Vibration Control of Damped Sandwich Beams," Jounal of Sound and Vibration, Vol. 246, No. 4, pp.653-677.

[61] Trindade, M.A., Benjeddou, A., and Ohayon, R., 1999, "Parametric analysis of the vibration control of sandwich beams through shear-based piezoelectric actuation," Journal of Intelligent Materials Systems and Structures, Vol. 10, No. 5, pp.377-385.

[62] Trindade, M.A. and Maio, C.E.B., 2006, "Passive vibration control of sandwich beams using shunted shear piezoelectric actuators," in IV Congresso Nacional de Engenharia Mecânica, Recife, ABCM.

[63] Trindade, M.A. and Maio, C.E.B., 2007, "Multimodal Passive Vibration Control of Sandwich beams with shunted shear piezoelectric materials," in 19th International Congress of Mechanical Engineering, Brasília, COBEM2007.

[64] Tsai, M.S., and Wang, K.W., 1999, "On the structural damping characteristics of active piezoelectric actuators with passive shunt," Journal of Sound and Vibration, Vol. 221, No. 1, pp.1-22.

[65] Viana, F.A.C. e Steffen, V.J., 2006, "Multimodal Vibration Damping through piezoelectric patches and optimal resonant shunt circuits, "Journal of the Brazilian Society of Mechanics Science and Engineering, Vol. XXVIII, No. 3, pp.293-310 